\title{
2. LE TERRAIN TECHNIQUE
}

Es gibt Menschen, welche die Geschichte des Fernsehens vor 4000 Jahren beginnen lassen und "pioneer color television experiments" mit Newtons Zerlegung des Lichts in Spektralfarben ansetzen ${ }^{1}$. Das Ziel der nun folgenden Darstellung ist bescheidener: Es geht um einen knappen Überblick über die technische Entwicklung des elektronischen Farbfernsehens. Es geht nicht darum, die zahlreichen Entwicklungspfade, welche die frühe Fernsehtechnik eingeschlagen hat, im Detail nachzuzeichnen. $\mathrm{Zu}$ dieser Thematik liegt eine ausreichend differenzierte Literatur vor $^{2}$. Vielmehr sollen die wichtigsten

1 Siehe Lynn A. YAEzel, Color it Confusing: A History of Color Television, in: Lawrence W. LiTCHY, Malachi C. Topping (Hg.), American Broadcast. A Source Book on the History of Radio and Television, New York 1976, S. 72-80. Als Quelle für diesen Unsinn gibt Yaezel das Buch von Richard W. Hubbell, 4000 Years of Television, New York 1942, an.

${ }^{2}$ Allgemein: August Karolus, Farbfernsehen, in: Zeitschrift für angewandte Physik 4 (1952) 8 S. 300-320; Erich ScHWARTZ, Farbfernsehen. Geschichtliches, augenblicklicher Stand, Tendenzen der Weiterentwicklung, in: Technische Hausmitteilungen des NWDR 6 (1954) 5/6 S. 105-126; Wilhelm Keller, Hundert Jahre Fernsehen 1883-1983, Berlin, Offenbach 1983; Helmut ScHöNFELDER, Fernsehtechnik im Wandel. Technologische Fortschritte verändern die Fernsehwelt, Berlin, Heidelberg, New York 1996; BuRNs, Television; Rick Marshall, The History of Television, Twickenham 1986; John WYVER, The Moving Image. An International History of Film, Television and Video, Oxford 1989; Raymond Fielding (Hg.), A Technological History of Motion Pictures and Television: An Anthology From the Pages of the Journal of the Society of Motion Pictures and Television Engineers, Berkeley 1967; KIssELoFf, The Box; INGLIS, Behind the Tube; Vladimir K. ZworykIN, George A. MorTon, Television. The Electronics of Image Transmission in Color and Monochrome, New York, London 1954; ABRAMson, Electronic Motion Pictures. Für Deutschland: Fritz BeLow, Zur Entwicklung des Fernsehens in Deutschland, in: FTZ (1950) 8 S. 301-308; Winfried B. LERG, Zur Entstehung des Fernsehens in Deutschland, in: Rundfunk und Fernsehen 15 (1967) 4 S. 349-375; Walter BRUCH, Vom Farbensehen zum Farbfernsehen, in: Bild der Wissenschaft 3 (1966) 7 S. 524-535; Gerhart GoEBEL, Das Fernsehen in Deutschland bis zum Jahre 1945, in: Archiv für Post- und Fernmeldewesen 5 (1953) 5 S. 259-293; Hans Pressler, Entwicklung des Farbfernsehens in Deutschland, in: Fernmeldetechnische Zeitschrift 1 (1948) 5 S. 99-102; Heidie RIEDEL, Fernsehen - Von der Vision zum Programm, Berlin 1985; William Uricchio (Hg.), The History of German Television, Sondernummer des Historical Journal of Film, Radio and Television 10 (1990) 2; Ders. (Hg.), Die Anfänge des deutschen Fernsehens. Kritische Annäherungen an die Entwicklung bis 1945, Tübingen 1991. Für die USA: LITCHY (Hg.), American Broadcast. Für Belgien: Radio et Télévision de la Belgique Francophone (RTBF) (Hg.), Nouveau l'histoire de la radio télévision en Belgique, Bruxelles 1986. Für Großbritannien: ABramson, History of Television; BuRns, Television; Tony CuRrIe, A Concise History of British Television, 1930-2000, Tiverton 2000; Graham Peter ScotT, British Television. An Insider's History, Jefferson, Lonson 2000. Für Frankreich: Pierre AlberT, André-Jean TudesQ, Histoire de la Radio-Télévision, Paris 1986; Christian BroCHAND, Histoire générale de la radio et de la télévision française, 2 Bde., Paris 1994; Marc Chauvierre, La télévision - hier, aujourd'hui et demain, Paris 1977. Für Skandinavien: Ib BonDEBJERG, Francesco BoNo (Hg.), Television in Scandinavia: History, Politics and Aesthetics, London 1996. Für Osteuropa: Joseph RoIzeN, Television in Eastern Europe and the Soviet Union, in: The Television Society Journal 11 (1965) 6 S. 136-141. Zudem gibt es zahlreiche 
Etappen, die zur Entwicklung desjenigen Systems geführt haben, das ohne Zweifel als "Referenzsystem « für alle weiteren technischen Farbfernsehentwicklungen gedient hat, kurz skizziert werden. Es handelt sich hierbei um das im Auftrag der US-amerikanischen Federal Communications Commission (FCC) entwickelte Farbfernsehsystem NTSC, das seinen Namen der FCC-Arbeitsgruppe »National Television System Committee« verdankt. Im AnschluB an die technikhistorische Darstellung des NTSC-Systems werden die europäischen Systemvarianten vorgestellt, wobei sich die Darstellung im wesentlichen auf die beiden letztlich erfolgreichen Varianten SECAM und PAL konzentriert.

Diese Begrenzung in der Darstellung der Farbfernsehentwicklung sollte nicht als retrospektive teleologische Deutung einer komplexen und offenen Entwicklungsgeschichte mißverstanden werden. Eine ausgiebige Würdigung jener alternativen Entwicklungspfade, beispielsweise der mechanischen Bilderzeugungsverfahren, die in enger Wechselwirkung mit dem elektrooptischen Verfahren diskutiert wurden, wäre jedoch die Aufgabe einer eigens diesem Thema gewidmeten technikhistorischen Darstellung.

Biographien und Autobiographien bedeutender Fernsehpioniere. Siehe beispielsweise ABraMson, Zworykin; Manfred voN ARDENNE, Entstehen des Fernsehens: persönliche Erinnerungen an das Entstehen des heutigen Fernsehens mit Elektronenstrahlröhren, Herten 1996; Russel W. Burns, John Logie Baird: Television Pioneer, London 2002; George Everson, The Story of Television: The Life of Philo T. Farnsworth, New York 1949; Donald G. GoDfreY, Philo T. Farnsworth: The Father of Television, Salt Lake City 2001; Peter C. Goldmark, Maverick Inventor: My Turbulent Years at CBS, New York 1973; Hildegard Karolus, August Karolus: ein Fernsehpionier. Die Anfänge des Fernsehens in Deutschland in Briefen, Dokumenten und Veröffentlichungen aus seiner Zusammenarbeit mit der Telefunken GmbH, Berlin 1923-1930, Berlin 1994; Frank Ronald Tiltman, Baird of Television. The Life Story of John Logie Baird, London 1933; Malcolm BAIRD, Television and Me. The Memoirs of John Logie Baird, Edinburgh 2004; Paul Schatzkin, The Boy Who Invented Television. A Story of Inspiration, Persistence and Quiet Passion, Silver Spring 2002; Michel AmOUDRY, René Barthélemy ou la grande aventure de la télévision française, Grenoble 1997. 
2.1. »It takes but the flip of the switch $\ll$. Die Farbfernsehentwicklung in den USA

\begin{abstract}
A beautiful girl in a colorful costume is lounging in an attractive room. Would you like to have her lounge in your living room? Now it is possible - by the way of color television. It takes but the flip of the switch, but this is no indication of the hours of mathematical development which went into this very complex system. The telephone was remarkable - the radio outstanding - television was a masterpiece - but color TV is an absolute miracle ${ }^{3}$.
\end{abstract}

Auch wenn diese Beschreibung den leichten Verdacht US-amerikanischer Technikeuphorie vermuten läßt, bezeugten auch die europäischen Fernsehingenieure dem Anfang der fünfziger Jahre in den USA entwickelten NTSCSystem technische Genialität. Worin lag die Genialität des NTSC-Systems? Um diese Frage beantworten zu können, müssen kurz die technischen Herausforderungen vorgestellt werden, vor denen die US-amerikanischen Ingenieure Anfang der 1950 er Jahre standen.

\title{
2.1.1. Die physikalisch-technischen Grundlagen ${ }^{4}$
}

Während beim Schwarzweißfernsehen lediglich die Helligkeit (Leuchtdichte) als zu übertragende Information existiert, müssen beim Farbfernsehen zwei zusätzliche Komponenten übertragen werden: der Farbton und die Farbsättigung. Die zu übertragende Bildinformation eines Farbfernsehbildes ist demnach wesentlich größer die eines s/w-Bildes, die dazu benötigte Bandbreite dementsprechend auch. Das Farbfernsehen baut, ähnlich wie die Farbfotografie, auf dem physikalischen Prinzip auf, daß man mit Hilfe der drei Grundfarben Rot, Grün und Blau durch sogenannte »additive Farbmischung « beliebige Farbtöne und Sättigungsgrade erreichen kann. Jeder Farbeindruck, den das menschliche Auge wahrnimmt, läßt sich demnach als eine von den drei Grundfarben erzeugte Mischung denken ${ }^{5}$. Wie läßt sich diese Erkenntnis technisch umsetzen? Um ein Farbbild zu übertragen, muß es zuallererst elektronisch erzeugt werden, das heißt, eine Farbkamera muß das aufzunehmende Bild in entsprechende elektrische Signale umwandeln. Dies geschieht durch

${ }^{3}$ Milton A. SizER, The Development of Colour-TV Signal, in: Radio Electronics 38 (1967) 1 S. 59.

${ }^{4}$ Bei der Beschreibung der physikalisch-technischen Grundlagen der Farbfernsehtechnik wird, soweit es die heute noch gültigen Erkenntnisse der Fernsehtechnik betrifft, das Präsens als Erzähltempus gewählt.

5 Einen allgemeinverständlichen Einstieg in die Farbenlehre und ihre Bedeutung für die Entwicklung des Farbfernsehens liefert Walter Bruch in seinem Kapitel über "Technikgeschichte des Farbfernsehens von seinen Uranfängen bis zum amerikanischen NTSC « in: BRUCH, RIEDEL, PAL - Das Farbfernsehen, S. 9-57. 
entsprechende Farbfilter, die das in das Objektiv der Kamera einfallende Licht in die drei Grundfarbenanteile zerlegen.

Das einfachste Farbfernsehsystem besteht demnach aus einer Kamera, die mit drei Aufnahmeröhren ausgestattet ist, die mit je einem Rot-, Grün- und Blaufilter versehen und zu einer Farbfernsehkamera zusammengefaßt sind. Die drei so aufnehmbaren Farbauszüge werden mittels einer lichtempfindlichen Schicht in elektrische Signale umgewandelt. Die drei Ausgangssignale dieser Kamera sind die den Grundfarbenanteilen entsprechenden Primärsignale für Rot, Grün und Blau, von denen jedes die gleiche Bandbreite beansprucht wie ein s/w-Signal. Auf der Empfängerseite werden diese drei Primärsignale nun dazu verwendet, sie mittels Projektion oder Spiegelung zur Deckung zu bringen. Das Prinzip der additiven Farbmischung ermöglicht schließlich die farbige Reproduktion der Bildvorlage auf dem Bildschirm ${ }^{6}$.

Dieses System der additiven Farbmischung wird auch heute noch bei Projektionsempfängern oder bei der Großprojektion von Farbfernsehbildern angewandt. Die Schwierigkeit besteht darin, die drei Farbauszüge hundertprozentig zur Deckung zu bringen. Die Lösung dieses Problems gelang dem bei der Radio Corporation of America (RCA) angestellten Entwicklungsingenieur A. N. Goldsmith im Jahre 1947. Sein Patent zur sogenannten shadowmask-Farbbildröhre gehört zu den folgenreichsten Erfindungen auf dem Gebiet der modernen Elektrotechnik. Dessen war sich der Präsident der RCA, David Sarnoff, schon wenige Jahre später bewußt, als er anläßlich der ersten öffentlichen Vorführung der shadow-mask-Röhre am 29. März 1950 in Washington verkündete:

Im Vergleich zu den großen Erfindungen im Bereich von Radio und Fernsehen der letzten fünfzig Jahre wird diese Farbbildröhre in die Annalen des Fernsehens als revolutionäre und epochemachende Entwicklung eingehen. Wenn Historiker gegen Ende des 20. Jahrhunderts die wichtigsten wissenschaftlichen Entdeckungen auflisten, wird diese Röhre in der Reihe der größten Ertindungen dieses Jahrhunderts stehen. Sie ist der Schlüssel zum Farbfernsehen und damit eine herausragende Leistung unserer Zeit ${ }^{7}$.

Die im Deutschen als »Lochmasken-« oder »Schattenmasken-Röhre« bezeichnete Farbbildröhre war nicht nur die Basis für die technisch-industrielle Durchsetzung des Farbfernsehens, sondern wurde auch zum Ausgangspunkt einer jahrzehntelangen Vormachtstellung der RCA auf dem Farbbildröhren-

6 Zur frühen Entwicklungsgeschichte der Fernsehkamera siehe ABramson, Electronic Motion Pictures sowie Max Artigalas, Michel Favreau, Les Caméras de télévision, in: Bulletin du Comité d'histoire de la télévision 18 (juin-juillet 1988) S. 29-62. Zur shadow-mask Farbfernsehröhre siehe D. Gabor, F. R. S. Member, P. R. Stuart, P. G. Kalman, A New Cathode-Ray Tube for Monochrome and Colour Television, in: Proceedings of the Institution of Electrotechnical Engineers 105 (1958) S. 581-606.

7 Zitiert nach Bruch, Riedel, PAL - Das Farbfernsehen, S. 39. 


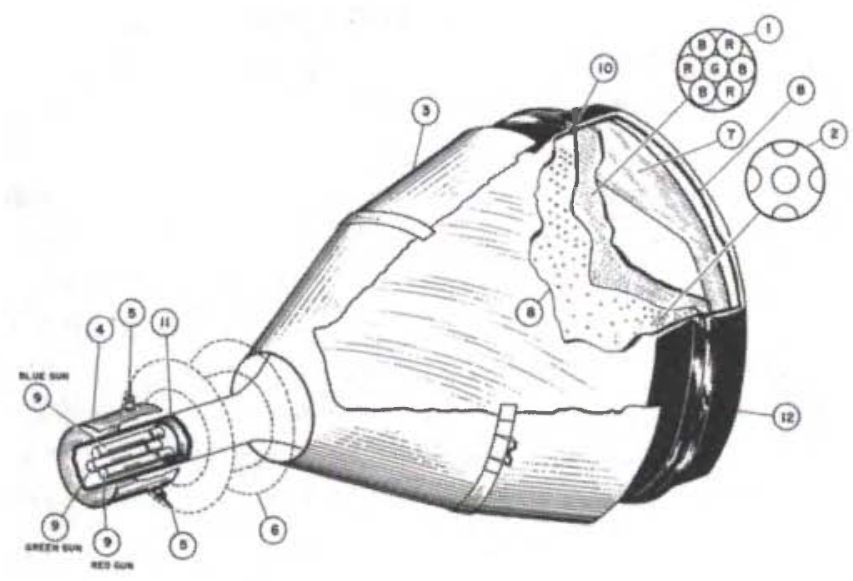

Abb. 1: RCA shadow-mask, aus: Archiv des Deutschen Museums München, Nachlaß Walter Bruch, Signatur NL 101, Nr. 122.

sektor. Worin bestand die Lösung des Problems? Einfach gesagt: In der getrennten Steuerung der drei Farbsignale durch eine Lochmaske.

Das Prinzip des Lochricht-Rasterverfahrens stammt aus der Fotografie. Die Idee, das farbige (polarisierte) Licht durch Elektronenstrahlen zu ersetzen, war der Grundgedanke der Lochmaskenröhre. Schon der deutsche Physiker Werner Flechsig meldete 1938 ein Patent zu einer "Kathodenstrahlröhre zur Erzeugung mehrfarbiger Bilder auf einem Leuchtschirm « an ${ }^{8}$, die Realisierung dieser Idee blieb aber den RCA-Ingenieuren um A. N. Goldsmith vorbehalten. Neben der Lochmaske und drei getrennten Kathodenstrahlen war die Beschichtung der Glasinnenseite der Empfängerröhre der dritte zentrale Bestandteil der Lochmaskenröhre. Der Schirm enthält in regelmäßiger Anordnung etwa 330000 Farbtripel, das heißt etwa 1000000 Farbleuchtpunkte. Die drei Strahlen der Elektronenstrahlkanonen werden so ausgerichtet, daß sie gemeinsam durch ein Loch in der Lochmaske gehen, um dann divergierend auf die zu einer Dreiergruppe zusammengefaßten rot, grün und blau leuchtenden Phosphorteilchen aufzutreffen. Der Abstand zwischen Schirm und Lochmaske beträgt $11,5 \mathrm{~mm}$, der Abstand zwischen den einzelnen Farbtripeln beträgt $0,74 \mathrm{~mm}^{9}$.

8 Werner Flechsig, Farbfernsehpatente aus dem Jahre 1938, in: Funkschau, 39 (1967) 17 S. 551-552. Flechsig (1900-1970), der zu dieser Zeit bei der Berliner Fernseh GmbH beschäftigt war, konnte diese Erfindung (Deutsches Reichspatent Nr. 736575 vom 12. Juli 1938) aber nicht zu einer produktionsreifen Röhre weiterentwickeln. Siehe BRUCH, RIEDEL, PAL - Das Farbfernsehen, S. 37.

${ }^{9}$ Eine allgemeinverständliche Beschreibung der Grundlagen der Farbröhrentechnik findet sich bei MüLLER, Farbfernsehen, sowie bei M. KouBEK, Zwanzig Jahre kompatibles Farbfernsehen. 
Das Aufbringen der Leuchtelemente (Phosphorteilchen), das Zusammenlöten der verschiedenen aus Hartglas gepreßten Glaseinzelteile zum kompletten Galskolben, in den die Lochmaske sowie die drei Kathodenstrahlkanonen eingebaut sind und schließlich die Evakuierung der gesamten Bildschirmröhre machen die Farbbildröhre zu einem der komplexesten massenindustriell hergestellten Konsumgüter überhaupt ${ }^{10}$. Die knappe Beschreibung dieser Technik, an deren Realisierung Hunderte von Spezialisten in mehreren Entwicklungslabors über Jahre gearbeitet haben, wird der Komplexität der Technik und der Bedeutung der Farbbildröhre für die Durchsetzung des Farbfernsehens mit Sicherheit nicht gerecht. Aus Gründen anderer Prioritätensetzung muß aber auf die unter technikhistorischen Gesichtspunkten interessante Beschreibung der Weiterentwicklung der Farbbildröhrentechnik verzichtet wer$\operatorname{den}^{11}$.

War das Aufteilen der Farbinformation in drei Farbsignale für die Kamera und den Empfänger so prinzipiell realisierbar, stellte dieses Prinzip die Fernsehingenieure vor ein anderes Problem: Das »3-Kanal-Verfahren« hat den Nachteil, daß bei der Übertragung (ob über Leitung, Rundfunk oder Satellit) die dreifache Kanalbreite für eine Farbfernsehübertragung benötigt wird. Praktisch bedeutet diese Tatsache, daß für die Übertragung eines Farbbildes bei einer Bildschirm-Zeilennorm von 625 Zeilen statt wie bislang $5 \mathrm{MHz}$

Der Stand der Farbfernsehtechnik - Rückblick und Ausblick, in: Radioschau 16 (1966) 4 S. 204-211.

10 Kaiser beschreibt im Jahre 1967 die Farbbildröhre als das wahrscheinlich komplizierteste »technische Teil«, das bislang in die Massenproduktion überführt werde. Rudolf KaISER, Farbtüchtige Fernsehübertragungswege der DBP, in: Der Ingenieur der Deutschen Bundespost 16 (1967) 3 S. 92-98. Karl Tetzner beschreibt die Farbbildröhre, in der alleine 15 Jahre Entwicklungsarbeit steckten, als »das wohl am schwierigsten zu fertigende Teil, das man jemals für den Gebrauchsgütersektor hergestellt hat «. Siehe Karl TeTzNER, Die Farbe im Fernsehen. Technik Wirtschaft - Organisation, in: Rundfunk und Fernsehen 15 (1967) 2 S. 118.

11 Eine Alternative zur Lochmaskenröhre war die sogenannte "Trinitron«-Röhre, eine EinstrahlChromatron-Röhre, die 1964 erstmals von der Firma Sony auf den Markt gebracht wurde. Statt der Lochmaske übernehmen hier Elektronenlinsen die Bündelung der Elektronenstrahlen. Diese Technik geht auf eine Idee des amerikanischen Nobelpreisträgers E. O. Lawrence zurück. 1953/54 stellte General Electric eine größere Zahl von Versuchsmodellen her, scheiterte jedoch an den Fertigungsschwierigkeiten, die mit der Realisierung dieser hochsensiblen Technik verbunden waren. Masaru Ibaku, Mitbegründer von Sony, gelang es nach jahrelangem mühevollen Experimentieren dennoch, dieses Prinzip in Form der Trinitron-Röhre umzusetzen. Die TrinitronRöhre war mit einem neuartigen Elektronensystem ausgestattet, das alle drei Kanonen in einem System vereinigte, und so den schwierigen Abgleich der drei Kanonen für deckungsgleiche Farbauszüge obsolet machte. Obwohl die Trinitron-Röhre als technisch beste Farbbildröhre gilt, blieb ihre Produktion auf die Firma Sony beschränkt und konnte sich gegenüber der leichter zu fertigenden shadow-mask-Röhre nicht durchsetzen. Siehe hierzu Ronald D. BROWN, Engineering for Colour, in: New Scientist 25. August 1966 S. 416-419. Eine detaillierte technische Beschreibung der Empfängerröhre findet man im Telefunken-Fachbuch »Farb-Fernseh-Technik«, hrsg. von der Telefunken AG, Fachbereich Röhren, Ulm 1966, S. 47-67 sowie bei Norbert MAYER, Technik des Farbfernsehens in Theorie und Praxis, Berlin 1967, S. 127-155. 
Bandbreite $15 \mathrm{MHz}$ benötigt werden. Da Mitte der fünfziger Jahre schon ein Mangel an verfügbaren Frequenzbändern existierte, stand man vor dem Problem, drei s/w-Kanäle für einen Farbfernsehkanal >opfern < zu müssen.

$\mathrm{Zu}$ diesem rundfunkpolitischen Problem im Bereich der internationalen Frequenzverwaltung ${ }^{12}$ gesellte sich eine Herausforderung ganz anderer Art: Die Einführung eines Farbfernsehsystems mit einer Bandbreite von $15 \mathrm{MHz}$ hätte bedeutet, daB der Fernsehzuschauer sich neben dem s/w-Gerät (mit einer Bandbreite von $5 \mathrm{MHz}$ ) einen zweiten Empfänger hätte kaufen müssen. War schon die Anschaffung eines s/w-Empfängers in den fünfziger Jahren ein Luxus der besonderen Art, wäre der Kauf eines zweiten, farbtüchtigen Empfängers wohl nur für eine verschwindend kleine Minderheit der Bevölkerung möglich gewesen. Im Gegensatz zur heutigen Zeit beanspruchte der Fernsehempfänger in den fünfziger Jahren den Status eines Luxusgutes mit einer >Halbwertzeit` von mindestens fünfzehn Jahren und einem symbolischen Wert vergleichbar mit dem eines Autos ${ }^{13}$.

Für den Farbfernsehempfänger stellte sich demnach eine Forderung, die man in der Rundfunkindustrie bereits aus der Geschichte des Schwarzweißfernsehens kannte ${ }^{14}$ : die Forderung nach Kompatibilität des Neuen mit dem Bestehenden. Beim Kauf eines neuen Farbempfängers wollte der Kunde mit diesem Gerät auch noch s/w-Sendungen sehen können. Eine Forderung, die angesichts des beim Beginn des Farbfernsehens sehr eingeschränkten farbigen Programmangebots mehr als berechtigt erschien. Umgekehrt soliten auch alle Besitzer eines $\mathrm{s} / \mathrm{w}$-Gerätes die in Farbe ausgestrahlten Sendungen in $\mathrm{s} / \mathrm{w}$ sehen können. Kompatibilität hieß in diesem Fall, daß für die Übertragung von Farbfernsehbildern nicht mehr Bandbreite benötigt werden durfte als bei der Übertragung von s/w-Bildern. Die Farbinformation (Farbton und Farbsättigung) mußte irgendwie in der Bandbreite von $5 \mathrm{MHz}$ untergebracht werden. Dies schien der Logik der Informationstheorie entgegen zu stehen und physikalisch nicht erfüllbar zu sein.

Einen Ausweg aus diesem Dilemma bot nicht eine neuartige Informationstheorie sondern die Trägheit des menschlichen Auges, oder, wertneutraler ausgedrückt, die physiologische Beschaffenheit des menschlichen Sehorgans

\footnotetext{
12 Siehe hierzu ausführlich Kap. 2.2. »The National Television System Committee«.

13 Noch in einem Bericht über das Farbfernsehen in Frankreich aus dem Jahr 1968 wird festgestellt, daB der hohe Preis der Farbempfänger ein wesentlicher Hemmschuh für die rasche Verbreitung des Farbfernsehens bedeute. Es heiBt: „Für den gleichen Preis kann man einen Kleinwagen kaufen, und damit ist die Frage für den Durchschnittsfranzosen überhaupt keine Frage: Das Auto geht vor火. Siehe N.N.: Farbfernsehen in Frankreich, in: Funk-Technik 23 (1968) 5 S. 154.

14 Die Probleme der Rundfunkindustrie, gerätetechnisch auf die Weiterentwicklung der Aufnahme- und Übertragungstechnik zu reagieren, lassen sich beispielhaft an der Herausbildung der unterschiedlichen s/w-Zeilennormen im europäischen Fernsehbetrieb veranschaulichen. Dieses Thema wird ausführlich am Beispiel der französischen 819-Zeilennorm und den Folgen dieser Entscheidung für die französische Rundfunkindustrie in Kapitel 2.3.1 diskutiert.
} 
und die Verarbeitung der Farbinformation im menschlichen Gehirn. Wahrnehmungsphysiologische Untersuchungen des menschlichen Auges hatten gezeigt, daß das Auflösungsvermögen für Farbunterschiede wesentlich geringer ist als für die Leuchtdichteunterschiede, also für die hell/dunkel-Unterschie$\mathrm{de}^{15}$. Das menschliche Auge hat gegenüber dem farbigen Detail ein bedeutend geringeres Auflösungsvermögen - der Reduktionsfaktor liegt zwischen 3 und 5 je nach Seh- und Verarbeitungsvermögen. Übertragen auf die Farbfernsehtechnik bedeutete dies, daß bei der Übertragung der drei Primärsignale mit voller Bandbreite überflüssige Farbinformation übertragen wird, die vom menschlichen Auge gar nicht wahrgenommen werden kann. Dieser Tatbestand legte den Gedanken nahe, das s/w-Bild (also das Leuchtdichtesignal) mit der gesamten Bandbreite von $5 \mathrm{MHz}$ zu übertragen, die Farbinformation dagegen mit bedeutend geringerer Bandbreite, etwa mit $1 \mathrm{MHz}$ zu übertragen. Ein beliebtes Bild zur Veranschaulichung dieses Prinzips ist der Vergleich mit dem Malbuch eines Kindes: Die bereits in Grauwerten vorgezeichneten Konturen lassen das wie auch immer ausgefärbte Objekt bereits deutlich erkennen ${ }^{16}$.

$\mathrm{Da} \beta$ die Unterbringung der Farbkomponenten im s/w-Band möglich war, wurde bereits 1946 im sogenannten »mixed-highs «-System der RCA erfolgreich demonstriert ${ }^{17}$. Da der obere Teil des Frequenzspektrums beim s/w-Signal relativ gering beansprucht wird, führte man bei $4,43 \mathrm{MHz}$ den soge-

15 Siehe Humberto Maturana u. a., Eine biologische Theorie der relativistischen Farbkodierung in der Primatenretina, in: Ders. (Hg), Erkennen. Die Organisation und Verkörperung von Wirklichkeit, Braunschweig 1982. Die erkenntnistheoretischen Folgerungen aus seinen neurobiologischen Forschungen präsentiert Maturana anschaulich in: DeRS., Was ist Erkennen?, München 1994. Der amerikanische Neurophysiologe William Calvin schildert die wahmehmungstheoretischen und bewuBtseinsphilosophischen Grundlagen der Sehforschung anschaulich in dem Kapitel "Aus bloßem Gehirn wird Geist: Die visuelle Welt wird zerlegt«. Siehe William H. CALvin, Die Symphonie des Denkens. Wie BewuBtsein entsteht, München 1995, S. 117-141.

16 Siehe MülLER, Farbfernsehen, S. 760.

17 Das »mixed-highs «-Verfahren war das erste in einer Reihe von Testverfahren, die man zur Reduktion der Bandbreite beim Farbfernsehen ausprobierte. Es folgten mehrere Systeme, die auf der Basis sequentieller, das heißt aufeinanderfolgender Übertragung der drei Primärsignale basierten. So das "field sequential system «, bei dem in aufeinanderfolgenden Teilbildern die Grundfarben übertragen wurden. Eine Weiterentwicklung stellte das »line sequential system《 dar, bei dem die Abtastnorm vom SchwarzweiBfernsehen übernommen und die Farbsignale zeilenweise übertragen wurden. Der nächste Schritt führte zum »dot sequential system «, zum Punktfolge-Verfahren. Die Umschaltung zwischen den Grundfarben erfolgte hier erstmals zwischen zwei aufeinander folgenden Bildpunkten. Die Erkenntnisse aus dem "dot sequential system« führten letztlich zu der für alle danach folgenden Systeme grundlegenden "System-Philosophie« des kompatiblen Farbfernsehens, wie sie im NTSC-System realisiert worden sind. Als Überblick siehe Koubek, Zwanzig Jahre kompatibles Farbfernsehen, S. 204 sowie ScHWARTz, Übersicht über die verschiedenen Farbfernsehsysteme, S. 487-504. Eine ausführliche technische Beschreibung findet sich bei INGLIS, Behind the Tube, S. $237 \mathrm{f}$. sowie vor allem bei den beiden Fernsehpionieren ZWORYKIN, MORTON, Television, S. 757-872. 


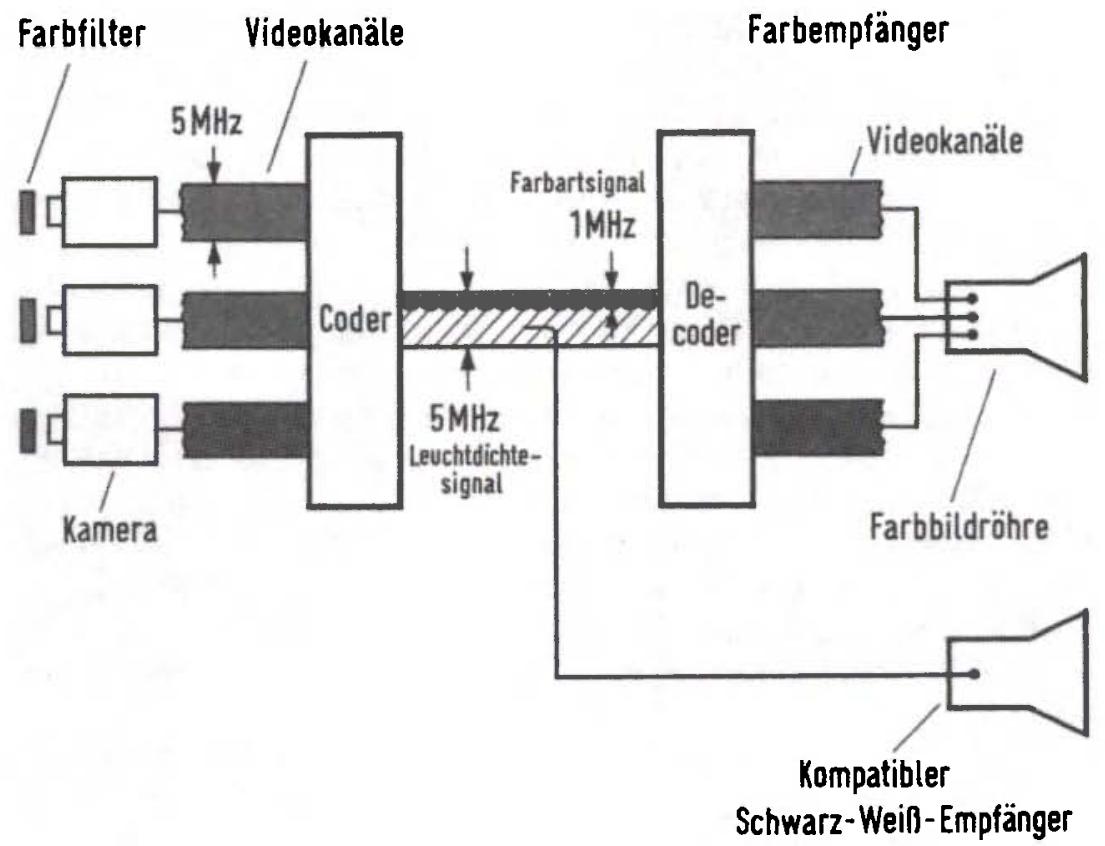

Abb. 2: Grundprinzip der Einkanalübertragung, aus: Archiv des Deutschen Museums München, Nachlaß Walter Bruch, Signatur. NL 101, Nr. 122.

nannten »Farbträger « in das Fernsehband ein, den man dann mit den beiden Farbkomponenten moduliert (Prinzip der Frequenzverkämmung oder »frequency interlace $\ll)$. Da sich die beiden Farbsignale wegen der oben beschriebenen Beschaffenheit des menschlichen Sehvermögens in reduzierter Bandbreite (etwa 0,5 und $1,5 \mathrm{MHz}$ ) realisieren lassen, gruppiert sich schließlich die gesamte Farbinformation um den Farbträger von 3 bis $5 \mathrm{MHz}$.

Die Unterbringung der Farbinformation in einem Signal, das nicht mehr Bandbreite benötigte als der s/w-Fernsehkanal, war also prinzipiell möglich. Die Herausforderung für die Fernsehtechniker bestand nun darin, die drei Farbsignale Rot, Grün und Blau in ein für den Fernsehrundfunk geeignetes Einkanal-Signal umzuwandeln. Genau dies ist die Aufgabe des eigentlichen Farbfernsehsystems. Abbildung 2 verdeutlicht das Grundprinzip aller kompatiblen Farbfernsehübertragungssysteme.

Die Aufgabe des Farbfernsehsystems wird hier klar: Die von der Farbkamera ausgegebenen Farbsignale Rot, Grün und Blau (R, G, B) müssen in einem "Coder « zu einem einzigen Signal mit einer Bandbreite von ca. $1 \mathrm{MHz}$ zusammengefaßt werden (Farbartsignal), das dem breitbandigen 5-MHz-Leuchtdichtesignal (Y-Signal genannt) aufmoduliert wird. Ist diese Forderung erreicht, spricht man von »senderseitiger Kompatibilität $«$ Im $» D e-$ 
coder« des Fernsehempfängers erfolgt die Rückwandlung des schmalbandigen Farbartsignals in die drei breitbandigen R-, G-, B-Signale zur Ansteuerung der Dreistrahl-Farbbildröhre. Wichtigste Zusatzbedingung für die Kodierung ist die Einhaltung der senderseitigen Kompatibilität, das heißt, der s/w-Empfänger muß das Leuchtdichtesignal aus dem übertragenen Gesamtsignal mit einem Minimum an Störwirkung wiedergeben können.

Bevor näher auf die unterschiedlichen technischen Übertragungsvarianten eingegangen wird, gilt es, folgendes festzuhalten: Das soeben beschriebene Prinzip der Einkanal-Farbfernsehübertragung gilt für alle später realisierten Farbfernsehsysteme gleichermaßen. Sowohl das amerikanische NTSC-System als auch das französische SECAM-System und das deutsche PAL-System basieren auf diesem Prinzip. Die drei Systeme unterscheiden sich lediglich in einem Punkt: in der Art der Modulation des Farbträgers. Die beiden zentralen Parameter der Entwicklung des Farbfernsehens, die Erzeugung eines Einkanal-Farbfernsehsignals sowie die Kompatibilität von Schwarzweißund Farbfernsehen, werden in allen später realisierten Farbfernsehsystemen beibehalten. 


\subsection{Das »National Television System Committee« (NTSC)}

Anfang der 1950er Jahre waren in den USA somit die wichtigsten wissenschaftlich-technischen Voraussetzungen für ein kompatibles elektronisches Farbfernsehsystem geschaffen worden. Obschon die USA das einzige Land der Welt waren, in dem das SchwarzweiBfernsehen nach 1945 einen rasanten Aufschwung erlebte, ist es erstaunlich, mit welchem Elan sich die US-amerikanische Rundfunkindustrie dem Thema Farbfernsehen zu einer Zeit widmete, in der das Geschäft mit den s/w-Empfängern erst langsam rentabel wurde. War (ist) es doch ein ungeschriebenes Gesetz der Rundfunkgeräteindustrie, eine die bestehende Produktpalette ablösende Gerätegeneration erst dann auf den Markt zu bringen, wenn sich eine Sättigungskurve im Absatz der ersten Generation andeutet ${ }^{18}$. Von einer Sättigung im Bereich der $\mathrm{s} / \mathrm{w}$-Empfänger konnte auf dem amerikanischen Markt um 1950 jedoch keine Rede sein. Der Impuls, sich intensiv mit der Entwicklung des Farbfernsehens zu beschäftigen, wurde von außen an die Rundfunkindustrie herangetragen, genauer gesagt von der Federal Communications Commission (FCC). Welches Interesse hatte die FCC an dieser frühzeitigen Beschäftigung mit dem Farbfernsehen? Über die Motive klärt uns ein Bericht von Stéphane Mallein auf, der im Januar 1951 im Auftrag der Radio Télédiffusion Française (RTF) auf Amerikareise war:

Contrairement à ce qu'on pourrait croire, ce ne sont pas les industriels, mais bien le F.C.C. qui a constamment poussé les recherches vers la couleur; on se rappelle qu'au début de 1949, la F.C.C. avait suspendu toute nouvelle allocation de fréquences pour la télévision et qu'elle avait mis à l'étude l'utilisation des fréquences supérieurs à $470 \mathrm{mc} / \mathrm{s}$, elle avait à ce moment bien précisé que cette étude serait conduite avec le souci de ménager l'avènement de la télévision en couleurs. Depuis cette date, chaque fois que l'industrie venait réclamer la mise en vigeur du nouveau plan, la F.C.C. ne manquait pas de rétorquer qu'il fallait d'abord qu'on lui proposât un système de couleurs valable ${ }^{19}$.

Die für die Frequenzvergabe in den USA zuständige Bundesbehörde FCC hatte also die Vergabe weiterer Frequenzen für den stark im Aufwind stehen-

18 Genau dies bewahrheitete sich ja auch im amerikanischen Fall. Der Absatz der Farbempfänger stieg erst sechs Jahre nach der Einführung des NTSC-Systems erheblich an, als eine flächendeckende Versorgung des Landes mit s/w-Empfängern realisiert war. »Wie das Beispiel der USA deutlich zeigt, wird die Einführung des FF erst dann aktuell, wenn sich - wirtschaftlich gesehen - auf dem monochromen Markt allmählich Sättigungserscheinungen zeigen«, bestätigte Walter Gerber 1965. Siehe Walter GERBER, Die Normung des Farbfernsehens in Europa, in: In ternationale Elektronische Rundschau 20 (1966) 9 S. 512. Zahllose Belege für die These des Sättigungsgrades liefert auch die Einführung neuer Radiomodelle im Rahmen der Transistorisierung der Empfänger. Siehe hierzu Fickers, "Der Transistor « als technisches und kulturelles Phänomen.

19 Stéphane Mallein, Rapport sur la mission effectuée à New-York et Washington du 14 au 23 décembre 1950, Paris, 31 janvier 1951, 10 maschinengeschriebene Seiten, hier S. 3, in: Archives nationales, Nr. 870714/Art. 14. 
den s/w-Fernsehrundfunk an die Bedingung geknüpft, sich vorher darüber im klaren zu sein, auf welchen Wellen- und Frequenzbereichen der zukünftige Farbfernsehbetrieb angesiedelt sein würde. Diese aus rundfunkpolitischer Perspektive vernünftige Überlegung setzte die US-amerikanische Rundfunkindustrie unter erheblichen Druck. Wollte man dem aufstrebenden s/w-Geschäft keine Grenzen in dem Sinne setzten, daß die Entstehung neuer Sender und damit zusätzlicher Programme verhindert wurde, mußte die Entwicklung eines tauglichen Farbfernsehsystems so schnell wie möglich realisiert werden. Woher hatte die FFC die Befugnis zu einer derart weitreichenden Entscheidung?

Um diese Frage zu beantworten, lohnt es, sich die Geschichte dieser Bundesbehörde vor Augen zu führen ${ }^{20}$. Bis 1927 gab es in den USA keinerlei staatliche Regelung im Bereich des Rundfunks. Rundfunkstationen konnten nach eigenem Gutdünken Sendefrequenzen besetzen und änderten diese nach Belieben. Um dieses Chaos zu beenden, wurde am 23. Februar 1927 im sogenannten »Radio Act« die »Federal Radio Commission« (FRC) geschaffen. Sie setzte sich aus fünf Kommissaren zusammen, die - bis heute - vom USamerikanischen Präsidenten vorgeschlagen und anschließend vom Senat bestätigt werden müssen ${ }^{21} .1934$ schuf der Kongreß mit dem »Federal Communications Act« die gesetzliche Grundlage für die Umbenennung und Kompetenzerweiterung der FRC in die "Federal Communications Commision« (FCC). Da die Sendefrequenzen in den USA als öffentliches Gut gelten (public airwaves), besteht die Aufgabe der FCC vornehmlich in der Regulierung inner- und zwischenstaatlicher Kommunkation »by regulating interstate and foreign commerce in communication by wire and radio ${ }^{22}$. Hans Joachim Kleinsteuber versucht in seinem Aufsatz "Regulierung des Rundfunks in den USA. Zur Kontrolle wirtschaftlicher Macht am Beispiel der FCC « nachzuweisen, daß sich in der FCC beispielhaft die typisch amerikanische Regulierungspolitik nach dem Muster der »countervailing powers« wiederspiegelt:

Den Traditionen des common law folgend, bezeichnet Regulation weniger ein von Gesetzen geleitetes hierarchisches und zielgerichtetes staatliches Handeln, sondern eher ein Prozedere [...] bei dem Konflikte als normal vorausgesetzt und deren staatlich moderierte Schlichtung in den Mittelpunkt gestellt werden. [...] Die Gerichtsähnlichkeit des regulativen Prozesses ist unverkennbar. Zwei Parteien streiten vor einer neutralen staatlichen Instanz, die selbst als Jury (die

20 Zur Geschichte und Bedeutung der FCC siehe diverse Beiträge in dem Sammelband von LiTCHY, Topping (Hg.), American Broadcast. Eine theoretische Auseindersetzung mit den Regulierungskompetenzen der FCC liefert der anregende Aufsatz von Hans Joachim KLEINSTEUBER, Regulierung des Rundfunks in den USA. Zur Kontrolle wirtschaftlicher Macht am Beispiel des FCC, in: Rundfunk und Fernsehen 44 (1996) 1 S. 27-50. Eine detaillierte technikhistorische Studie zur Rolle der FCC bei Standardisierungsfragen liefert Hugh R. SLOTTEN, Radio and Television Regulation: Broadcast Technology in the United States, 1920-1960, Baltimore 2000.

21 Die »Commissioner« sind für fünf Jahre gewählt und »unabsetzbar «.

${ }^{22}$ Kleinsteuber, Rundfunkregulierung in den USA, S. 32. 
fünfköpfige FCC) konzipiert ist. [...] Man könnte sagen, die FCC repräsentiert eine verstaatliche Variante der Selbstregulierungsbedarfe aus der Rundfunkindustrie ${ }^{23}$.

Auch wenn Kleinsteubers Thesen im Bezug auf die Kontrolle wirtschaftlicher Macht (FCC als Antitrust-Behörde im Bereich der Rundfunkindustrie) plausibel erscheinen, sollte die von ihm konstatierte »ausgeprägte Bürgerbeteiligung und die Öffentlichkeit des Regulierungsprozedere« nicht überbewertet werden. Lawrence Litchy hat bereits 1976 nachgewiesen, daß Idee und Wirklichkeit oftmals voneinander abweichen und der »Transparenz« daher praktische Grenzen gesetzt sind. Litchy betont, daß die Politik des FCC stark vom jeweiligen Hintergrund der einzelnen Kommissare gefärbt war: »[...] the personal experience, education, occupational background, and governmental philosophy of the members of the FRC and FCC have overtly influenced the direction and emphasis of the agency's policies. [...] Many important decisions or changes were the result of a scrusader by one commissioner ${ }^{24}$.

Versuchen wir, Kleinsteubers Überlegungen am Beispiel der Kontroverse zwischen den beiden Protagonisten CBS und RCA um den Farbfernsehstandard zu verifizieren. Wie bereits beschrieben, hatte die FCC 1948 alle weiteren Lizenzvergaben im Bereich des Fernsehens »because of the confusing surrounding engineering standards and color television ${ }^{25}$ eingefroren. Diese als $\gg$ TV freeze $\ll^{26}$ in die Geschichte eingegangene Entscheidung der FCC war mit der Aufforderung des FCC an die Entwicklungsfirmen verbunden, der FCC ihren jeweiligen Forschungsstand im Farbfernsehbereich im September 1949 vorzulegen. Sie leitete somit das ihr zur Verfügung stehende Rechtsverfahren des »rule-making process « ein, das mit der Veröffentlichung von Vorschlägen beginnt, der anschließenden Möglichkeit von Einsprüchen und Anhörungen (hearings) fortgesetzt wird, bis das Verfahren mit der Verkündung eines »report and order « abgeschlossen wird.

Im Oktober und November 1949 fanden die sogenannten »hearings« statt. Die RCA stellte hier erstmals ihr s/w-kompatibles Farbfernsehsystem vor. Die erreichte Bildqualität des RCA-Systems blieb jedoch deutlich hinter der des elektro-mechanischen Systems der CBS zurück. Schwachpunkt des RCASystems war der Farbempfänger, beziehungsweise die Farbfernsehröhre. Während der Anhörungen kündigte die RCA jedoch an, sie werde innerhalb

23 Ibid. S. 29, 32 und 33.

${ }^{24}$ Lawrence W. LiTCHY, The Impact of FRC and FCC Commissioners' Backgrounds on the Regulation of Broadcasting, in: DerS., MaLACHI, American Broadcast, S. $630 \mathrm{f}$. Litchy macht in der Zeit von 1927 bis 1976 sieben Epochen aus. Jede dieser Epochen ist seiner Meinung nach durch den personellen »background« der fünf Commissioner bestimmt, wobei mal Techniker, mal Politiker, mal Juristen die Politik der FCC maBgeblich bestimmten.

25 Ibid. S. 627.

26 Zum Thema »TV freeze« siehe BoDDY, Fifties Television, S. 42-64. 
der nächsten sechs Monate eine neuartige Farbfernsehröhre vorstellen können, welche die bisherigen Schwächen des Systems kompensieren werde. In diesen sechs Monaten gelang den Ingenieuren und Technikern der RCA tatsächlich, was viele Experten nicht für realisierbar hielten:

Over the winter, more than fifty scientists and engineers at the Princeton Labs, supported by technical and administrative staff, worked with engineers at RCA's CRT (cathode ray tube, A.d.V.) plant in Lancaster, Pennsylvania, to make a demonstration model. By the end of March 1950, as promised, RCA showed the FCC its color picture tube in an receiver similar to those consumers bought for a black-and-white television. More than any other component of the system, the shadow-mask CRT made color television a practical household technology ${ }^{27}$.

Trotz dieser Leistung seitens der RCA-Ingenieure legte die FCC nach Abschluß der Anhörungen im Mai 1950 das von der CBS vorgestellte bildsequentielle elektromechanische Farbfernsehsystem, welches mit dem bestehenden s/w-System nicht kompatibel war, als US-amerikanischen Standard fest $^{28}$. Die RCA reagierte mit einem Protest beim obersten amerikanischen Gerichtshof, und auch das National Bureau of Standards (NBS) legte im Juli 1950 einen Untersuchungsbericht vor, in dem sie das RCA-System in 12 von 18 Vergleichspunkten als dem CBS-System überlegen beschrieb.

Parallel zu den Aktivitäten der FCC hatte sich bereits im Januar 1950 auf Anregung der Radio Manufacturers Association (RMA) ein zweites National Television System Committee (NTSC) gebildet, das - so die Hoffnung der zahlreich vertretenen Rundfunkfirmen - wie schon 1940 die entscheidende Wendung (diesmal pro RCA) im Kampf um einen einheitlichen Standard bringen sollte ${ }^{29}$. Das, was dem zweiten NTSC in den nur knapp drei Jahren von seiner Zusammensetzung (Januar 1950) bis zur Übernahme des vor-

27 IEEE Milestones, Monochrome-Compatible, Electronic Color Television, 1946-1953, in: http://www.iece.org/organizations/history_center/milestones_photos/colortvtech.html (Juni 2006). 28 In dem »Second Report on Color Television« wurde das CBS-System zum amerikanischen Standard erklärt. Siehe Peter Goldmark u. a., Color Television - USA Standard, in: Proceedings of the IRE 39 (1951) S. 1288-1313. Siehe auch Heinwig LANG, 40 Jahre Farbfernsehen nach dem Prinzip der konstanten Luminanz, in: Fernseh- und Kino-Technik 49 (1995) 1-2 S. 35-40.

29 Das erste National Television System Committee war am 17. Juli 1940 auf Anregung der RMA unter der Leitung von Dr. W. R. G. Baker von der General Electric Company gegründet worden. Schon damals waren alle großen Namen der Branche vertreten: Bell Telephone Labs, CBS, Don Lee Broadcasting System, DuMont Labs Inc., Farnsworth Television and Radio Corp., General Electric Company, Hazeltine Service Corp., John V. L. Hogan, Hughes Tool Company, IRE, Philco Corp., RCA, Stromberg-Carlson Telephone Mfg. Company, Television Productions und Zenith Corp. Am 2. Mai 1941 verabschiedete die FCC schlieBlich die vom NTSC vorgeschlagenen Normen für das SchwarzweiBfernsehen in den USA (u.a. die 525-Zeilennorm) und kündigte den Beginn des kommerziellen SchwarzweiBfernsehens für den 1. Juli 1941 an. Siehe Abramson, The History of Television 1880 to 1941 , besonders Kapitel $11 »$ The first NTSC: $1940-1942 \ll$, S. 257-273. 
geschlagenen Farbfernsehstandards durch die FCC (17. Dezember 1953) gelang, wurde retrospektiv von vielen Fachleuten als $»$ Glanzstück $\aleph^{30}, » \mathrm{MaB}$ aller Dinge ${ }^{31}$ oder »eine der genialsten Erfindungen des Fernsehens ${ }^{32}$ bezeichnet. Für die meisten US-amerikanischen Hersteller spielte das Argument der Kompatibilität mit dem bestehenden s/w-System die zentrale Rolle bei der Entscheidung, das RCA-System zu unterstützen.

In diesem zweiten National Television System Committee fanden sich über hundert Physiker, Ingenieure und Techniker aller großen US-amerikanischen Fernsehfirmen zusammen (auch CBS war beteiligt), um die Idee eines kompatiblen, vollelektronischen Farbfernsehsystems zu realisieren. Die entscheidenden Anregungen, die schließlich zur erfolgreichen Entwicklung des Systems führten, kamen von den Mitarbeitern der Hazeltine Corporation unter Leitung von D. B. Loughlin. Loughlin faBte seine Modifikationen an dem RCA-System unter dem Namen »Constant Luminance System« zusammen.

Es bestand im wesentlichen aus zwei Modifikationen des RCA-Systems ${ }^{33}$ : 1) Statt des Signals aus den "gemischten Höhen« (mixed highs) wurde ein breitbandiges Helligkeits- bzw. Luminanzsignal $Y$ gebildet. Dieses Luminanzsignal war die gewichtete Summe der drei Farbwertsignale R (Rot), G (Grün) und B (Blau). 2) Als Buntheits- oder Chrominanzsignal wurden nicht die drei Farbwertsignale selbst, sondern die Differenzen (die so genannten Farbdifferenzsignale) zur Modulation des Farbträgers verwendet.

Was bedeuteten diese beiden Veränderungen konkret? Farbphysiologische Untersuchungen hatten gezeigt, daB das menschliche Auge farbiges Licht unterschiedlicher Intensität je nach Farbton in unterschiedlicher Helligkeit wahrnimmt. In einem weißen Lichtstrahl trägt beispielsweise die Grünkomponente (G) 59\%, die Rotkomponente (R) $30 \%$ und die Blaukomponente (B) nur $11 \%$ zu dem erzeugten Helligkeitseindruck bei. Mit anderen Worten: blaue Farben erscheinen dem Auge dunkler als rote und diese wiederum dunkler als grüne ${ }^{34}$. Addiert man also die drei Farbwertsignale in diesem anteiligen Verhältnis zueinander, so erhält man ein resultierendes Helligkeitsoder Luminanzsignal (Y-Signal), das wie das Bildsignal des SchwarzweiBfernsehens alle Informationen über die Helligkeitsverteilung des Bildes enthält. Formelhaft ausgedrückt bedeutet dies: $Y=0,3 \mathrm{R}+0,59 \mathrm{G}+0,11 \mathrm{~B}$.

\footnotetext{
30 Walter KaISER, Von der Nachrichtenübermittlung zur Telekommunikation, in: Hans-Joachim BraUn, Walter KaISER, Energiewirtschaft, Automatisierung, Information, Propyläen Technikgeschichte Bd. 5, Berlin 1997, S. 394.

31 Norbert MAYER, Farbfernsehübertragung mit gleichzeitiger Frequenz- und Amplitudenmodulation des Farbträgers (FAM-Verfahren), in: Rundfunktechnische Mitteilungen 4 (1960) S. 238.

32 MüLLER, Die internationalen Bemühungen um eine europäische Farbfernsehnorm, S. 228.

33 LANG, 40 Jahre Farbfernsehen, S. $38 \mathrm{f}$.

34 Fritz JAESCHKE, Europa vor der Einführung des Fabfernsehens, in: Umschau in Wissenschaft und Technik 66 (1966) 8 S. 259.
} 
Dieses Y-Signal wird in der gleichen Technik, vor allem mit der gleichen Bandbreite wie in der $s / w-T e c h n i k$ übertragen und liefert am Empfangsort ein normales s/w-Bild. Dieser Prozeß der Signalmischung bedeutet mathematisch eine Koordinatentransformation, bei der das dreiparametrige System R, G, B in ein neues, linear davon abhängiges System überführt wird ${ }^{35}$.

Zur vollständigen Definition eines Bildpunktes in diesem neuen System sind wiederum drei Koordinaten erforderlich, von denen bislang nur eine mit dem Y-Signal festgelegt ist. Zwei weitere, welche die benötigten Farbinformationen definieren müssen, fehlen noch. An diesem Punkt setzten Loughlins Überlegungen zu den Farbdifferenzsignalen an. Er schlug vor, auch für die Farbsignale eine Koordinatentransformation vorzunehmen, indem anstelle der Farbwertsignale R, G, und B die Farbdifferenzsignale U = $B-Y$ und $V=R-Y$ übertragen werden. Über eine recht einfache Matrix lassen sich so die drei Farbwertsignale in die beiden Farbdifferenzsignale R-Y und B-Y umwandeln. Die beiden Farbdifferenzsignale sind dann für den Farboder Chrominanzanteil des zu übertragenden Bildes allein verantwortlich. Da es sich bei den beiden Farbdifferenzsignalen zudem um die beiden Farbkomponenten handelt, die im Vergleich zur Grünkomponente die geringeren Helligkeitsanteile besitzen, kann so wertvolle Bandbreite eingespart werden. Loughlin zeigte auch, wie die beiden Farbdifferenzsignale im Empfänger über eine Dematrix wiedergewonnen werden konnten. Schließlich läßt sich das dritte Farbsignal (Grün) aus den beiden Farbdifferenzsignalen B-Y und R-Y und dem Helligkeitssignal (Y-Signal) ableiten. Durch Addition des Luminanzsignals erhält man schließlich die für die Farbbildröhre erforderlichen Farbwertsignale R, G und B.

Die Ingenieure der RCA waren von Loughlins Vorschlägen so begeistert, $\mathrm{da} B$ sie diese unmittelbar in ihr System übernahmen ${ }^{36}$. Als letztes Problem blieb den Experten des NTSC die Frage der Übertragungsart der Farbdifferenzsignale zu klären, beziehungsweise die Frage der Kombination von Chrominanz- und Luminanzkanal zu beantworten. Wie bereits erwähnt, wurde die insgesamt zur Verfügung stehende Übertragungsbandbreite von $5 \mathrm{MHz}$ in vollem Umfang von dem Helligkeitssignal beansprucht. Die zusätzliche Farbinformation mußte daher gemeinsam mit dem Y-Signal im gleichen Kanal untergebracht werden. $\mathrm{Da} B$ dies prinzipiell möglich war, hatten die RCA-Ingenieure bereits 1946 mit dem sogenannten »mixed highs «-Verfahren gezeigt.

Die Kombination des "mixed highs«-Verfahrens mit den Vorschlägen von Loughlin (»constant luminance«) gelang den Ingenieuren der Philco Corpora-

35 Fritz JAEsCHKE, Übersicht über den Stand der Fernsehtechnik, in: Jahrbuch des elektrischen Fernmeldewesens 18 (1967) S. 195.

36 Die spannende Phase der Arbeit am Farbfernsehen im zweiten NTSC beschreibt der langjährige Leiter der RCA Forschungslaboratorien in Princeton, George H. BROwN in seinem Buch "... and Part of Which I Was 
tion. Dort schlug man vor, die beiden Chrominanzkomponenten (R-Y und B-Y) der Farbträgerfrequenz in Quadraturmodulation aufzuprägen, die dann dem Luminanzsignal additiv zugesetzt wird ${ }^{37}$. Bei der Quadraturmodulation wird der Farbhilfsträger in zwei »senkrecht« zueinanderstehende Komponenten aufgespalten (Phasenverschiebung von $90^{\circ}$ ). Diese werden in Gegentaktmodulation mit je einem der beiden Farbdifferenzsignale amplitudenmoduliert.

Wegen der Analogie zum »Newtonschen Farbkreis« nennt man die Darstellung des Farbträgers in der komplexen Ebene auch "Chrominanzebene«. In Folge dieser Analogie kann man nach Schönfelder von einem besonders anschaulichen Modulationsverfahren sprechen: Die Farbträgeramplitude bestimmt die Farbsättigung und die Farbträgerphase den Farbton ${ }^{38}$. Trotz der »Genialität« dieser Lösung sollte sich herausstellen, daß die Abhängigkeit des Farbtons von der Farbträgerphase das System in der Praxis relativ anfällig für unschöne Farbverzerrungen machen sollte, da es während der Übertragung des Farbsignals leicht zu Phasenverschiebungen kommen konnte. Doch dazu später.

Es wären noch zahlreiche weitere Details notwendig, wollte man der komplexen Struktur des NTSC-Systems gerecht werden, doch soll an diesem Punkt nicht weiter auf die technisch-wissenschaftlichen Grundlagen eingegangen werden ${ }^{39}$. Festzuhalten bleibt, daß es den führenden US-amerikanischen Rundfunkunternehmen in einer beeindruckenden Gemeinschaftsleistung gelungen war, ein den selbstgesteckten Anforderungen zur Genüge reichendes kompatibles, vollelektronisches Farbfernsehsystem zu entwickeln.

Neben den bereits geschilderten Entwicklungen im Bereich der Übertragungstechnik und Farbkodierung war die bei der RCA entwickelte Farbbildröhre das dritte zentrale Standbein, das letztlich den Durchbruch des NTSCSystems ermöglichte. Am 17. Dezember 1953 legte die FCC das vom National Television System Committee vorgeschlagene Farbfernsehsystem als neuen US-amerikanischen Farbfernsehstandard fest und revidierte damit die

37 SCHÖNFELDER, Fernsehtechnik im Wandel, S. $33 \mathrm{f}$.

38 Ibid. S. 36. Zur Bestimmung einer Farbe in der Farbmetrik sind immer die beiden Informationen des Farbtons und der Farbsättigung notwendig.

39 Siehe hierzu u.a. MAYer, Technik des Farbfernsehens in Theorie und Praxis; Helmut ScHöNFELDER, Die Farbsynchronisierung beim NTSC-Verfahren, in: Archiv der elektrischen Übertragung 18 (1964) 6 S. 355-370; JAESCHKE, Übersicht über den Stand der Farbfernsehtechnik; Gordon J. KING, Beginner's Guide to Colour Television, London 1973; Günther KrolL, Farbfernsehen - leicht verständlich, Hamburg 1967. B.W. OsBORNE, Colour Television Reception and Decoding Technique, London 1968; C. R. G. Reed, Principles of Colour Television Systems, London 1969; Richard THEILE, Die Entwicklung der kompatiblen Farbfernsehtechnik unter besonderer Berücksichtigung der verschiedenen Vorschläge für die Übertragung der Farbart-Signale (Farbträgermodulation), in: Rundfunktechnische Mitteilungen 9 (1965) 5 S. 241-250; F. W. de VRIJER,: Übertragungssysteme für das Farbfernsehen, in: Philips Technische Rundschau 27 (1966) 4 S. 85-99. 
1950 getroffene Entscheidung für das CBS-System. Als Beginn für den Start der Farbfernsehsendungen nach dem neuen NTSC-System wurde der 22. Januar 1954 bestimmt.

Die organisatorischen und institutionellen Rahmenbedingungen, in denen sich die Fernsehtechnik in Europa entwickelte, unterschieden sich deutlich von denen in den USA. Als man 1954 das Farbfernsehen in den USA einführte, war man in den meisten europäischen Ländern gerade damit beschäftigt, einen stabilen s/w-Fernsehfunk auf die Beine zu stellen. An Farbfernsehen dachte - zumindest im Bereich der praktischen Realisierung - zu dieser Zeit niemand. Dennoch vollzogen sich Ende der vierziger, Anfang der fünfziger Jahre in Europa einige entscheidende Entwicklungen im Bereich der Fernsehtechnik, die für das künftige Farbfernsehen von zentraler Bedeutung waren. Ohne eine grobe Kenntnis dieser Vorgeschichte sind viele spätere Entwicklungen nicht nachvollziehbar. In einem kurzen Exkurs sollen daher jene rundfunktechnischen und -politischen Faktoren beschrieben werden, die für die Entwicklung des Farbfernsehens in Europa von entscheidender Bedeutung waren, die jedoch im kollektiven Gedächtnis, das eindeutig durch die politisierte PAL-SECAM-Kontroverse überfärbt wurde, untergegangen sind. 


\subsection{Das Problem der Zeilennorm in der Entwicklung des Schwarzweißfernsehens}

Der Beginn regelmäBig ausgestrahlter Fernsehensendungen in Europa fällt in die 1930 er Jahre. Am 15. Januar 1936 wurde in Berlin der regelmäBige Fernsehbetrieb des "Fernsehsenders Paul Nipkow « aufgenommen, das englische BBC-Versuchsprogramm wurde am 2. November des gleichen Jahres aufgenommen. Trotz aller technischen Anstrengungen und politischen Ambitionen ist Winfried Lerg zuzustimmen, daß der Fernsehbetrieb bis zum Beginn des Zweiten Weltkrieges in einem "Stadium des permanenten Experiments verharrte $^{40}$. Zudem war es - zumindest in Deutschland und Frankreich - ein Medium ohne Publikum. Die für die Durchsetzung des Hörfunks so wichtige "popularisierende Amateurbewegung « und "Detektor-Ära« fehlte dem Fernsehen, wofür natürlich in erster Linie die wesentlich komplexeren Anforderungen im Bereich der Empfängertechnik verantwortlich waren ${ }^{41}$. Auch setzte man in Deutschland ähnlich wie beim Beginn des Hörfunks auf das Konzept "gemeinschaftlicher « Mediennutzung. Das Konzept des "Saalfunks« für das Radio der frühen zwanziger Jahre fand sein Pendant in den 15 öffentlichen Fernsehstellen Berlins des Jahres 1936 oder ihren Pendants in der französischen Hauptstadt ${ }^{42}$.

Ein wenig anders sah die Situation in GroBbritannien aus. Hier vollzog sich die Streuung der Empfangsgeräte in private Haushalte wesentlich schneller

40 Winfried B. LERG, Zur Entstehung des Fernsehens in Deutschland, in: Rundfunk und Fernsehen 15 (1967) 4 S. 349-375, S. 363.

41 Siehe Monika Elsner, Thomas Müller, Peter M. Spangenderg, Der lange Weg eines schnellen Mediums: Zur Frühgeschichte des deutschen Fernsehens, in: URICcHIo (Hg.), Die Anfänge des deutschen Fernsehens, S. 153-207. Aus literaturhistorischer Perspektive wird hier eine mentalitätshistorische Darstellung der Suche des Fernsehens nach seiner »medialen Identität« geliefert. Der diskursanalytische Zugriff ist eine interessante Erweiterung der klassischen rundfunkhistorischen Interpretationen. Siehe auch Bruce A. MURRAY, Christopher J. WickHAM (Hg.), Framing the Past. The Historiography of German Cinema and Television, Illinois 1992.

42 Zur Geschichte des Saalfunks siehe Winfried B. LERG, Rundfunkpolitische Infrastruktur, in: BAusch (Hg.), Rundfunk in Deutschland, Bd. 1, S. $177 \mathrm{f}$. Eine innovative kulturgeschichtliche Analyse der Frühphase des Hörfunks liefert die Arbeit von Carsten LENK, Die Erscheinung des Rundfunks. Einführung und Nutzung eines neuen Mediums 1923-1932, Opladen 1997. Lenk teilt seine Studie in drei thematische Bereiche: die Verortung des Rundfunks (Öffentlichkeit versus Heim), die Freizeit als Radiozeit und schlieBlich die Durchdringung der Lebenswelt durch das neue Medium. Dadurch gelingt ihm eine alltagsgeschichtliche Rekonstruktion des Umgangs mit dem neuen technischen Apparat, die auch in dem von Inge MarBolek und Adelheid von Saldern herausgegebenen Sammelband »Radiozeiten. Herrschaft, Alltag, Gesellschaft (1924-1969) Potsdam 1999, ertragreich fortgefuhrt wird. Absolut unverständlich ist daher, wie eine sich als »Einfuhrung* titulierende Darstellung der Rundfunkgeschichte in Deutschland, wie sie Konrad Dussel vorgelegt hat, in den klassischen programm- und institutionengeschichtlichen Dimensionen der Rundfunkgeschichtsschreibung verharr. Siehe Konrad Dussel, Deutsche Rundfunkgeschichte. Eine Einführung, Konstanz 1999. 
und ermöglichte so ein demokratischeres Nutzungsverhalten. Am 2. November 1936 eröffnete die BBC ihr Fernsehversuchsprogramm, und gegen Ende des Jahres 1937 zählte man in England bereits 1600 Empfangsgeräte. Im Gegensatz zu Deutschland und Frankreich war das neue Medium hier »auf dem besten Weg, sich mit seinem Publikum zu treffen ${ }^{43}$. Bei Ausbruch des Zweiten Weltkrieges, mit dem alle Fernsehaktivität ad acta gelegt wurden, wurde die Gesamtempfängerzahl in England auf 20000 bis 25000 Stück geschätzt.

Auch die USA spielte bei der experimentellen Erforschung der Fernsehbildübertagung eine wichtige Rolle. Ende 1932 strahlten hier bereits 35 Versuchsstationen Fernsehsendungen aus. Ein erstes regelmäßiges und öffentliches Fernsehprogramm wurde aber erst 1939 anläßlich der Weltausstellung in New York eröffnet ${ }^{44}$.

In Frankreich startete das Fernsehversuchsprogramm 1935 mit der Aufstellung eines $10 \mathrm{~kW}$-Senders auf dem Eiffelturm. 1937 wurden täglich von 20 bis 20.30 Uhr Versuchssendungen ausgestrahlt, für die der spätere Generaldirektor der staatlichen Rundfunkorganisation Radio et Télévision Française (RTF), Wladimir Porché, verantwortlich war. Im Gegensatz zu England erlagen die Fernsehaktivitäten in Frankreich nicht komplett den Kriegseinwirkungen, sondern sie fanden in gewisser Weise mit den Programmen des »Fernsehsenders Paris« ab September 1943 eine Fortsetzung ${ }^{45}$.

Kennzeichnend für die Frühphase der Fernsehentwicklung und für die weitere Betrachtung von zentraler Bedeutung ist die Tatsache, daß die technische Entwicklung des Fernsehens größtenteils in einem national begrenzten Kontext stattfand und das Entwicklungsniveau und -tempo maßgeblich von einem eng begrenzten Personenkreis bestimmt wurde. Letzteres hat dazu geführt, daß die Technikgeschichtsschreibung des Fernsehens - besonders für die Frühphase des SchwarzweiBfernsehens - hauptsächlich eine Geschichte der "großen Männer«, der »Fernsehpioniere« ist. So mangelt es nicht an Biographien über diese Pioniere und an autobiographischen Selbstzeugnissen aus dieser Pionierzeit ${ }^{46}$, eine Überblicksdarstellung aus der Feder eines Technik-

${ }^{43}$ LERG, Entstehung des Fernsehens in Deutschland, S. 370. Zur Geschichte des frühen Fernsehrundfunks in GroBbritannien siehe auBerdem Asa BrigGs, BBC - The First Fifty Years, Oxford 1985; Burns, British Television; Abramson, History of Television 1880 to 1941.

44 Andreas Fickers, Presenting the $>$ Window to the World to the World. Competing Narratives of the Presentation of Television at the World's Fairs in Paris (1937) and New York (1939). Vortrag, gehalten auf dem World's Fair Symposium in San Francisco, April 2005.

45 Zur Geschichte des Fernsehsenders Paris siehe Thierry Kubler, Emmanuel Lemieux, Cognacq-Jay. La télévision française sous l'occupation, Paris 1990; Petra Truckendanner, Der Fernsehsender Paris. Deutsch-französisches Okkupationsfernsehen (1942-1944), in: Rundfunk und Geschichte 25 (1999) 2/3 S. 107-118; Dies., Fernsehsender Paris: Deutsch-französisches Okkupationsfernsehen, Diss. Universität Salzburg 1998.

46 Siehe für Frankreich Amoudry, René Barthelemy; Chauvierre, La Télevision. Ein unveröfentlichtes Manuskript (96 maschinengeschriebene Seiten) der Memoiren von Henri de France 
historikers ist dagegen bis heute nicht existent. Diese Forschung im "pionierzentrierten « und nationalen Kontext ${ }^{47}$ hatte zur Folge, daB eine große wirtschaftliche Konkurrenz zwischen den anfangs meist kleinen Firmen herrschte, und da $B$ sich in den verschiedenen Ländern unterschiedliche Fernsehsysteme durchsetzten. Erst nachdem sich unterschiedliche Systeme in den einzelnen Ländern etabliert hatten, wurde man sich einer Problematik bewußt, die dem neuen Medium Fernsehen inhärent war: Es stellte sich das Problem der Kompatibilität unterschiedlich genormter Systeme. Waren die Normparameter des Hörfunks wesentlich durch die Festlegung der Sendefrequenz und der Modulations- und Demodulationsart der Tonsignale bestimmt, kam beim "Bildfunk « ein neues Kriterium hinzu: die Definition der Auflösung der Bildinformation, festgelegt durch die Zeilenzahl des Bildschirms und die Taktfrequenz des Bildwechsels.

Die Grundidee der elektronischen Bildübertragung, die Zerlegung eines Bildes in nacheinander zu übertragende Bildpunkte, die anschlieBende Übertragung dieser Bildpunkte in Zeilen sowie die Umsetzung bewegter Vorgänge in Reihenbilder, geht auf den Berliner Paul Nipkow (1860-1940) zurück $^{48}$. Auch hier war die Trägheit des menschlichen Sehvermögens Voraus-

befindet sich in den Archivbeständen des Comité d'histoire de la télévision, Institut national de l'audiovisuel (INA) in Bry-sur-Marne. Für die USA steht beispielhaft die Autobiographie des RCA-Ingenieurs George H. BRown, ... and Part of Which I Was; Harold B. LAw, The Shadow Mask Color Picture Tube: How it Began - An Eyewitness Account of its Early History, in: IEEE transactions on electronic devices 23 (1976) 7 S. 752-759; ABRAMson, Zworykin; GoDFREY, Philo T. Farnsworth, sowie die historische Aufarbeitung des RCA-Ingenieurs Andrew INGLIS, Behind the Tube. Für Großbritannien: BuRNs, John Logie Baird; Maurice Exwoon, John Logie Baird: 50 Years of Television, London 1976. Für Deutschland: Die unveröffentlichten Lebenserinnerungen von Walter Bruch befinden sich im Archiv der Hochschule Mittweida, an der Walter Bruch in den dreiBiger Jahren studierte. BRUCH, Die Fernseh-Story, Stuttgart 1969; GoEBEL, Das Fernsehen in Deutschland bis zum Jahre 1945; Pressler, Farbfernseh-Erinnerungen, in: Rundfunktechnische Mitteilungen 17 (1973) 5 S. 205-213. Hans RiNDFLEISCH, Technik im Rundfunk. Ein Stück deutscher Rundfunkgeschichte von den Anfängen bis zum Beginn der achtziger Jahre, Norderstedt 1985; Erich SchwarTz, Farbfernsehen. Geschichtliches, augenblicklicher Stand, Tendenzen der Weiterentwicklung, in: Technische Hausmitteilungen des NWDR 6 (1954) 5/6 S. 105-126; SchöNFELDER, Fernsehtechnik im Wandel; LANG, 40 Jahre Farbfernsehen; Fritz Schröter, Fritz SchILgEN, Technik ist kein Geheimnis - der Blick hinter den Bildschirm, Teil 1: Fernsehgeschichte, in: Archivdienst der Telefunken GmbH, 15. August 1959, 27 Seiten.

${ }^{47}$ Diese national fixierte und auf die glorreichen Pioniere ausgerichtete Betrachtung hat deutliche Spuren in einer nationalistisch überhöhten populärwissenschaftlichen Geschichtsschreibung und hagiographisch geprägten Erinnerungskultur hinterlassen. Es gibt kaum ein an der frühen Fernsehentwicklung beteiligtes Land, welches nicht sdie Erfindung، des Fernsehens für sich reklamiert! Siehe hierzu Andreas FicKers, Frank KessLer, Techno-Nationalist Tales of Glory and Failure: Writing the History of Inventions in Early Film and Television, Vortrag auf der Tagung "Media in Transition 4: The Work of Stories«, MIT Boston, Mai 2005.

48 Am 6. Januar 1884 wurde Nipkows Erfindung als »elektrisches Teleskop« zum Deutschen Reichspatent angemeldet. Zentrales Element des Nipkow-Systems war die später nach ihm be- 
setzung für die technische Umsetzung: Wenn der Aufbau der einzelnen Bildzeilen und somit die Bildfrequenz höher als 10 Bildwechsel pro Sekunde ist, verschmelzen diese Bilder in unserer Wahmehmung zu einem einzigen stehenden oder bewegten Bild (Daumenkino-Prinzip) ${ }^{49}$.

Schon früh erkannte man, daß eine Verbesserung der Bildqualität mit einer Erhöhung der Zeilenzahl verbunden war. Allerdings waren der mechanischen Lochscheibentechnik hier Grenzen gesetzt. Erst die Arbeiten des aus der Sowjetunion stammenden Ingenieurs Wladimir Zworykin führten aus dieser technologischen Sackgasse heraus. Zworykin, der ab 1919 in den USA bei Westinghouse und ab 1929 bei der Radio Corporation of America arbeitete, hatte schon als Schüler des russischen Erfinders Boris Rosing am angesehenen Laboratorium des russischen Militärinstituts in St. Petersburg an der elektronischen Bildabtastung gearbeitet ${ }^{50} .1923$ meldete er eine elektronische Fernsehkamera namens »Ikonoskop « zum Patent an. Auch in anderen Ländern arbeitete man zu dieser Zeit an der Idee, die Braunsche Röhre als elektronischen Bildschreiber einzusetzen ${ }^{51}$. Auch wenn die Bildqualität der ersten elektronischen Fernsehbilder anfangs deutlich unter derjenigen der perfektionierten Nipkow-Scheiben lag, wurde allen Fernsehingenieuren bald klar, da $B$ hier die Weichen für eine grundlegende Änderung des Entwicklungspfades gestellt wurden. »For all intents and purposes«, so Albert Abramson in seiner Studie über die frühe Fernsehentwicklung, "the disclosure of the Iconoscope

nannte Nipkow-Scheibe. Als rotierende Scheibe mit 24 spiralförmig angeordneten Löchern zerlegte sie das zu übertragende Bild in Bildpunkte, was eine zeilenförmige Abtastung und Übertragung der Bildinformation ermöglichte.

49 Das europäische Fernsehbild besteht aus 625 Zeilen bei einer Bildfrequenz von $50 \mathrm{~Hz}$, das heiBt 25 Bildern pro Sekunde zusammen. In den USA und einigen anderen Ländern besteht das Fernsehbild aus 525 Zeilen, was jedoch durch die höhere Bildfrequenz von $60 \mathrm{~Hz}$ (30 Bilder/Sekunde) ausgeglichen wird. Für das menschliche Auge sind diese Unterschiede nicht wahmehmbar.

50 Braun, Anfänge des Fernsehens, S. 162.

51 In Deutschland arbeiteten Forscher wie Manfred von Ardenne und Siegmund Loewe seit 1925 an dieser Idee. Von Ardenne gelang 1930 in seinem Berliner Laboratorium die erste vollelektronische Fernsehübertragung. Siehe hierzu Manfred vON ARDENNE, Fernsehempfang. Bau und Betrieb einer Anlage zur Aufnahme des Ultrakurz-Wellen-Fernsehrundfunks mit Braunscher Röhre, Berlin 1935, sowie die beiden Kapitel »Grenzen der Mechanik« und »Aufbruch der Elektronik « in: RIEDEL, Fernsehen, S. 27-103. Einen Überblick zum damaligen Stand der deutschen Fernsehtechnik mit Aufsätzen aller FernsehgröBen (Ardenne, Schröter, Karolus, Banneitz etc.) bietet der 1937 von Fritz Schröter herausgegebene Sammelband „Fernsehen. Die neuere Entwicklung insbesondere der deutschen Fernsehtechnik«, Berlin 1937. Eine Synthese der Resultate der frühen Fernsehtechnik lieferte 1934 der Ingenieur R. Thun in seinem Buch $»$ Fernsehen und Bildfunk. Die allgemeinen Grundlagen, der gegenwärtige Stand«, Stuttgart 1934. In den USA war es der »Fernsehpionier« Philo T. Farnsworth, der zwischen 1928 und 1936 eine brauchbare Bildsondenröhre entwickelte. Siehe Everson, The Story of Television, sowie GodFrEY, Philo T. Farnsworth. 
marks the beginnung of the age of electronic television ${ }^{52}$. Wie später bei der Farbfernsehentwicklung war auch beim Schwarzweißfernsehen die Entwicklung der Kameratechnik von entscheidender Bedeutung. Die technischen Möglichkeiten der Bildzerlegung bestimmten letztlich die anschließenden Techniken der Bildübertragung und Bildrekonstruktion im Empfänger. Der System- bzw. Netzwerkcharakter der Fernsehtechnik wird an diesem Beispiel besonders deutlich.

Der Übergang von der mechanischen zur elektronischen Bildabtastung war der entscheidende Technologiesprung, der die schrittweise Erhöhung der Zeilenzahl und damit der Bildqualität zur Folge hatte ${ }^{53}$. In allen Ländern, die an der technologischen Entwicklung des Fernsehens beteiligt waren, experimentierte man an der optimalen Zeilenzahl. In Großbritannien legte man sich schließlich am 2. Februar 1937 auf 405 Zeilen fest, in Frankreich bevorzugte man 1937 das 455-Zeilensystem, während in Deutschland im August 1938 441 Zeilen als Standard definiert wurden. In den USA einigte man sich am 20. März 1941 auf 525 Zeilen, obschon man bei der Weltausstellung 1939 in New York mit dem 441-Zeilensystem begonnen hatte.

Tabelle 1: Europäische s/w-Zeilennormen vor der Harmonisierung von 1961.

\begin{tabular}{lcccc}
\hline \multicolumn{5}{c}{ Europäische Fernsehnormen in den Bändern I und III } \\
Zeilensystem & Videoband (MHz) & Bild/Tonabstand (MHz) & Bildmodulation & Tonmodulation \\
\hline 405 (GB) & 3 & $-3,5$ & Positiv & AM \\
625 (EBU) & 5 & 5,5 & Negativ & FM \\
625 (B) & 5,5 & 6 & Positiv & AM \\
625 (OIR) & 6 & 6,5 & Negativ & FM \\
819 (F) & 10 & 11,15 & Positiv & AM \\
819 & 5 & 5,5 & Positiv & AM \\
\hline
\end{tabular}

Der nationalen Normfestlegung ging meist ein erbitterter Kampf der an der Entwicklung des Fernsehens beteiligten Firmen voraus. Jedes Unternehmen vertrat mit seinem System eine eigene Zeilennorm. Das Beispiel der USA demonstriert diese Konkurrenz. Die RCA zeigte im April 1936 erste Demonstrationen seines 343-Zeilensystems bei 30 Bildwechseln/Sekunde. Auch "Farnsworth Television « begann in Philadelphia mit Versuchen des gleichen Systems. In Los Angeles präsentierte Harry Lubcke von der »Don Lee

\section{Abramson, History of Television 1880 to 1940, S. 199.}

53 .Technische Voraussetzung dieser Entwicklung war die Realisierung leistungsstarker hochfrequenter Senderöhren, da die Erhöhung der Bildwechsel- und Zeilenzahlen wegen der erforderlichen Frequenzbandbreiten nicht mehr auf Lang- oder Mittelwellensendern realisiert werden konnte. Schon Mitte der zwanziger Jahre wurde von F. Aigner, A. Esau und F. Schröter die Verwendung von Uitrakurzwellen zur Ausstrahlung von Fernsehbildern vorgeschlagen. Siehe RINDFLEISCH, Technik im Rundfunk, S. 82f, sowie ScHÖNFELDER, Fernsehtechnik im Wandel, S. 8-11. 
Broadcasting System Corporation« ein »high-definition« Fernsehsystem mit 300 Zeilen bei 24 Bildern pro Sekunde. Die »Philco Radio and Television Corporation « startete am 18. Juni 1936 gar eine Serie regulärer Programme mit einem 345-Zeilensystem bei 30 Bildern pro Sekunde. Das Television Committee der Radio Manufacturs Association (RMA) schlug der Federal Communications Commission im Juni 1936 ein 450-Zeilensystem mit einer Halbbildfrequenz von $60 \mathrm{Hertz}$ vor $^{54}$. Gleiches könnte man für Frankreich, Großbritannien oder Deutschland aufzählen ${ }^{55}$. Hinzu kam, daß die unterschiedliche Zeilennorm nicht der einzige Unterschied zwischen den diversen $\mathrm{s} / \mathrm{w}$-Fernsehsystemen war. Es gesellten sich unterschiedliche Vorschläge für die Bandbreite des Übertragungskanals und die Abstände zwischen Bild- und Tonträgerfrequenz hinzu.

Kurz: es gab ungefähr so viele Systemvorschläge wie Rundfunkunternehmen! Wie konnte man dieser Entwicklung begegnen, die der grundlegenden Voraussetzung der »Netzwerktechnologie« Rundfunk zu widersprechen schien? Die Einführung unterschiedlicher Zeilensysteme im Schwarzweißfernsehen bedeutete ja, daß ein Fernsehgerät nur jene Programme empfangen kann, die von einem Sender mit den entsprechenden Systemcharakteristika ausgesendet wurden. Dies wiederum hätte eine inhaltliche Einschränkung des Programmangebots sowie eine starke geographische Einschränkung der Empfangsgebiete zur Folge gehabt.

War man vor Beginn des Zweiten Weltkrieges vor allem damit beschäftigt, einen einheitlichen nationalen s/w-Standard auf die Beine $\mathrm{zu}$ stellen, traten nach 1945 langsam die Probleme des internationalen Programmaustausches ins Bewußtsein der Verantwortlichen der nationalen Rundfunkanstalten ${ }^{56}$. Die BBC nahm den regelmäßigen Fernsehbetrieb am 1. September 1946 wieder auf - mit dem altbewährten 405-Zeilensystem. In Frankreich dagegen entfachte sich eine lebhafte Auseinandersetzung um die Zeilennorm, die es lohnt, näher betrachtet zu werden. Warum? Zum einen, weil das Resultat dieser Auseinandersetzung, die Festlegung des 819-Zeilensystems im Jahre 1948, in der darauffolgenden Debatte um die Farbfernsehnorm eine wichtige Rolle spielte. Zum anderen, weil sich an dieser Auseinandersetzung im nationalen Kontext beispielhaft studieren läßt, was sich bei der Farbfernsehdiskussion auf internationaler Ebene zu wiederholen schien: die personelle Verflechtung und das institutionelle Ineinandergreifen technischer, wirtschaftli-

54 Siehe hierzu Abramson, History of Television 1880 to 1941 , S. $230 \mathrm{f}$.

55 Paul Bellac, Problèmes internationaux de la télévision - la normalisation, in: UIR Bulletin Mensuel (Juli 1949) 282, S. 397-404; Corey P. Carbonara, HDTV- A Historical Perspective, in: Lou Casabianca (Hg.), The New TV. A Comprehensive Survey of High Definition Television, Westport 1992, S. 3-26.

56 Siehe W. ReIchel, Auf dem Wege zur europäischen Fernsehnorm, in: Elektrotechnik 3 (1949) 6 S. 177-182. 
cher und politischer Akteure und Institutionen bei Standardisierungsprozessen im Rundfunkbereich.

Tabelle 2: $\quad$ Europäische s/w-Zeilennormen nach der Harmonisierung 1961

\begin{tabular}{lccccc}
\hline 625-Zeilen & $\begin{array}{c}\text { Europäische Fernsehnormen in den Bändern IV und V } \\
\text { Videoband } \\
(\mathrm{MHz})\end{array}$ & $\begin{array}{c}\text { Bild/Tonabstand } \\
(\mathrm{MHz})\end{array}$ & Bildmodulation & Tonmodulation & $\begin{array}{c}\text { Farbträger } \\
(\mathrm{MHz})\end{array}$ \\
\hline EBU & 5 & 5,5 & Negativ & FM & 4,43 \\
GB & 5,5 & 6 & Negativ & FM & 4,43 \\
OIRT & 6 & 6,5 & Negativ & FM & 4,43 \\
F & 6 & 6,5 & Positiv & AM & 4,43 \\
\hline
\end{tabular}

\subsection{1. "La bataille des standards - Das 819-Zeilensystem in Frankreich}

Im Gegensatz zu Großbritannien, wo während des Zweiten Weltkrieges sämtliche Aktivitäten im Bereich des öffentlichen Fernsehrundfunks lahmgelegt wurden, erlebte Frankreich unter der deutschen Besatzung eine kuriose Fortführung der Fernsehaktivitäten ${ }^{57}$. War im Mai 1941 ein Befehl des Höheren Nachrichtenführers, der für Fernmeldewesen zuständigen Dienststelle des Militärbefehlshabers in Frankreich, zum Abbau der französischen Sendeanlagen auf dem Eiffelturm zwecks »Rohstoffbeschaffung « ergangen, wurde dieser Befehl aus politisch-ökonomischen und militärischen Gründen letztlich nicht umgesetzt. Im Gegenteil: am 20 Mai 1942 befahl das Oberkommando der Wehrmacht die Wiederinbetriebnahme des Senders, der als »Fernsehsender Paris« in die Geschichte des Rundfunks eingehen sollte. Konzentrierte sich die propagandistische Mission des Senders auf die Unterhaltung in Paris stationierter deutscher Soldaten, weist Petra Truckendanner zu Recht darauf hin, daß diesem Projekt handfeste wirtschaftliche Interessen zugrunde lagen:

Neben der angestrebten Errichtung eines Programmbetriebes zur Unterhaltung und Zerstreuung der verwundeten Soldaten in den Lazaretten spielten deutscherseits langfristige wirtschaftliche Ziele im Hinblick auf die durchaus schon absehbare Entwicklung des Fernsehens nach dem Krieg und die Durchsetzung deutscher Normen eine Rolle. So bestand der Plan, im Rahmen einer >europäischen Television< nach Italien nun auch Frankreich für die 441-Zeilen-Norm zu gewinnen, um der deutschen Elektroindustrie damit für die Nachkriegszeit eine gute Ausgangslage zu verschaffen ${ }^{58}$.

Interessant sind in diesem Zusammenhang besonders die personellen Verflechtungen, die vor, während und nach dem Krieg im Bereich der Fernseh-

57 Siehe hierzu Kubler, Lemieux, Cognacq-Jay 1940; Truckendanner, Der Fernsehsender Paris; Jacques Poinsignon, Fernsehsender Paris, in: Bulletin du Comité d'histoire de la télévision 21 (mars-avril 1990) S. 31-62.

58 Truckendanner, Der Fernsehsender Paris, S. 108. 
technik bestanden. Bereits 1936 kam es zwischen Telefunken und der Compagnie des compteurs ( $\mathrm{CdC}$ ) zu einem strategischen Abkommen im Bereich der Fernsehentwicklung. Ein Forschungsabkommen mit einer Laufzeit von zehn Jahren sah unter anderem gemeinsame Forschungs- und Entwicklungsarbeiten im Bereich der Fernsehröhren (lkonoskope), der elektronischen Verstärker, der Sender im 2-Meter-Wellenbereich und im Bereich der hochauflösenden Fernsehempfänger ( 1000 Zeilen) vor ${ }^{59}$. Mit dem Abkommen verbunden war auch ein Patentaustausch zwischen beiden Unternehmen ${ }^{60}$.

Verständlich wird dieses ambitiöse Abkommen, wenn man sich die weltweiten Konzentrationsbemühungen in der elektrotechnischen Industrie vor Augen führt, die auch vor der noch jungen Fernsehbranche nicht haltmach$\operatorname{ten}^{61}$. Auf deutscher Seite (Telefunken) war der Fernsehfachmann Fritz Schröter, auf französischer Seite waren René Barthélemy und Paul Mandel an der Verhandlung des Abkommens beteiligt. Die guten deutsch-französischen Beziehungen auf diesem Gebiet ermöglichten beim Betrieb des »Fernsehsenders Paris« eine nahezu mühelose Fortführung der früheren Aktivitäten. Thierry Kubler und Emmanuel Lemieux gehen in ihrer Darstellung »Cognacq-Jay 1940. La télévision française sous l'occupation « sogar so weit, der harmonischen Zusammenarbeit zwischen deutschen und französischen Forschern "Freimaurer-Charakter « zu bescheinigen ${ }^{62}$. Hier kommt ein Punkt erstmals zur Sprache, der im Laufe der Darstellung an Gewicht gewinnen wird, nämlich die Bedeutung der Forscherpersönlichkeit in der Entwicklung der Technik oder bei der Aushandlung technischer Normen und Standards.

Die Geschichte des Fernsehsenders Paris ist zudem ein markantes Beispiel für das »Ich diente nur der Technik «-Rechtfertigungsverhalten vieler im Nationalsozialismus engagierter Techniker und Wissenschaftler ${ }^{63}$. Nicht nur auf

59 Kubler, Lemeux, Cognac Jay, S. $56 \mathrm{f}$.

60 Dieses Abkommen dient in den fünfziger Jahren als Basis einer erneuten Zusammenarbeit zwischen Telefunken und der Compagnie Générale de Télégraphie sans Fil (C.s.F.). Siehe hierzu Andreas Fickers, Coopération-confrontation-cohabitation: les relations entre la CSF/CFT et AEG-Telefunken en matière de brevets et licences de télévision en couleur dans les années soixante, in: Revue d'Allemagne 37 (2005) 1 S. 29-43.

61 In Großbritannien war es vor allem die "Marconi Telegraph and Wireless Company«, die zahlreiche kleinere Unternehmen, so auch das des britischen Fernsehpioniers John L. Baird, "sschluckte*. In den USA waren es General Electric und die RCA, welche ihren technologischen Vorsprung zu einer marktbeherrschenden Stellung ausbauen konnten.

62 »L'argument peut paraitre faible, pourtant plusieurs raisons historiques et industrielles expliquent le rapprochement entre les membres de la $\mathrm{CdC}$ et la Telefunken. En premier lieu, la fameuse sfranc-maconnerie des chercheurs lie particulièrement Barthelémy et Schröter; ils s'estiment et échangent leurs points de vue depuis le début des années 30 . Leur personnalité, alliant rigeur et austérité, tend a les rapprocher.« KUBLER, LemIEux, Cognacq-Jay, S. 58.

${ }^{63}$ Siehe Dirk BRÜNDEL, Museum für Verkehr und Technik (Hg.), Ich diente nur der Technik: sieben Karrieren zwischen 1940 und 1950, Berliner Beiträge zur Technikgeschichte und Industriekultur Bd. 14, Berlin 1995. 
deutscher Seite, wo die Rundfunktechniker im Dienste nationalsozialistischer Propagandaarbeit standen, ist dieses Muster zu erkennen, sondern auch in Frankreich, wo sich die Techniker des Fernsehsenders Paris zugleich als "Opfer und Förderer« des unter deutscher Leitung betriebenen Fernsehbetriebs verstanden ${ }^{64}$. Nach dem Zweiten Weltkrieg änderte sich an der guten deutsch-französischen Zusammenarbeit auf dem Gebiet des Fernsehens zunächst wenig. Nun war es die französische Compagnie des compteurs, die zum Arbeitgeber deutscher Fernsehingenieure wie Fritz Schröter, Richard Theile oder Kurt Diels wurde. Sogar der ehemalige »Sonderführer« des Fernsehsenders Paris, Kurt Hinzmann, der 1945 in Abwesenheit von einem englischen Militärgericht wegen Spionage zu fünfzehn Jahren Haft verurteilt wurde, diente ab 1946 als Verbindungsmann zwischen Telefunken und der Compagnie des compteurs. Von Fritz Schröter war Hinzmann in wundersamer Weise zum »Ingenieur « befördert worden. Insgesamt siebzehn Telefunken-Leute arbeiteten ab 1946 für die CdC in einem Grundlagenlabor in Corbeville bei Paris. 1946 rechtfertigte der »chef du service de télévision «, André Ory, dieses Verhalten gegenüber einem Journalisten mit den Worten: "Je considère cette affaire comme favorable au développement de la télévision française. Je trouve tout à fait normal que nous fassions ce que font les Américains et les Anglais. La venue des Allemands peut avancer d'un an la mise en route d'un programme commercial ${ }^{65}$.

Dies war ganz im Sinne der deutschen Techniker, wie Kurt Diels am 29. Oktober 1946 in seinem Bewerbungsschreiben an das zuständige Amt für Industrieproduktion der französischen Besatzungsmacht in Baden-Baden formulierte: »Étant donné que les recherches ne sont pas permises en Allemagne, mes ingénieurs et moi aimerions mieux travailler ailleurs, parce que

64 Klaus WinkER, Fernsehen unterm Hakenkreuz. Organisation, Programm, Personal, Köln u. a. 1994, S. 285f. In Frankreich mußte lediglich ein Mitarbeiter des technischen Stabs des Fernsehsenders Paris eine dreimonatige Haft verbüßen. Marc Chauvierre, neben Barthélemy und de France einer der führenden Fernsehentwickler in Frankreich, war bis 1940 Directeur Général von Radio-Cité, bis er unter Pétain zum "Directeur de Service cinématographique de la télévision " ernannt wurde. In der Darstellung von Kubler und Lemieux wird Chauvierre mit den Worten zitiert: "Par la suite, j'ai demandé la révision de mon procès, et j'ai été totalement blanchi. Je ne m'occupais que de technique à la radio«. KuBler, Lemieux, Cognacq-Jay, S. 202. Nach dem Krieg versuchte Chauvierre vergeblich, eine verantwortungsvolle Position im französischen Fernsehen wiederzuerlangen. Er wurde zu einem der vehementesten Bekämpfer der 819-Zeilennorm und seines Protagonisten Henri de France. Ein von Chauvierre im Mai 1985 an das Comité d'histoire de la télévision abgetretener Schriftwechsel zur 819-Zeilen-Problematik erlaubt einen detaillierten Einblick in diese Geschichte. Auch in seinem 1977 erschienen Buch »La télévision. Hier, aujourd'hui et demain« übte Chauvierre heftige Kritik an der französischen Fernsehpolitik, der er einen nationalistischen "Isolationismus « vorwarf.

65 So Ory in einem Interview mit dem Journalisten Francis Lamotte, das am 12. Dezember 1946 in der Tageszeitung "Le Pays« erschien. Siehe Kubler/Lemieux, Cognacq-Jay, S. 205. 
nous aimons trop notre métier pour l'abandonner définitivement ${ }^{66}$. Keinen dieser Herren sollte dieses Schicksal ereilen - im Gegenteil. Einige blieben bis zur Pension im Dienste der CdC, andere machten in der Bundesrepublik Karriere. So beispielsweise Richard Theile, der Leiter des Instituts für Rundfunktechnik in München wurde, oder Kurt Hinzmann, der Leiter des Fernsehens des Südwestfunks, später des Bayerischen Rundfunks wurde. Schröter und Diels verließen die CdC 1952, um Aufbauhilfe für das spanische Fernsehen unter Franco zu leisten.

Bereits im März 1945 wurde der experimentelle Betrieb des französischen Fernsehens in den Studioanlagen der »rue Cognacq Jay« wiederaufgenommen. Der regelmäßige Fernsehbetrieb mit dem von den Deutschen übernommenen 441-Zeilensystem konnte aber erst im Oktober 1947 beginnen. Man startete mit einem Programm von 12 Stunden pro Woche ${ }^{67}$. Allerdings sollte diese Regelmäßigkeit bald einer neuen Zeilendiskussion zum Opfer fallen. Schon während des Krieges hatten einige Forscher in Frankreich an der Erhöhung der Zeilennorm für das Schwarzweißfernsehen gearbeitet. René Barthélemy entwickelte im Labor der CdC ein 1015-Zeilensystem, Henri de France experimentierte in seinem Labor der Radio-Industries in Lyon an einem 819-Zeilensystem und einige Ingenieure um Jean-Louis Delvaux und Yves Delbord in der Compagnie Française Thomson-Houston (CFTH) brachten ein 729-Zeilensystem zustande.

Die hohe Zeilenzahl brachte technisch einige Probleme mit sich, die im Detail nicht diskutiert werden können. Zwei Faktoren sprachen aber aus französischer Perspektive Mitte der vierziger Jahre für die Einführung eines hochauflösenden Schwarzweißfernsehens: Zum einen die höhere Bildqualität $^{68}$, zum anderen - und dies war der gewichtigere Grund - der Schutz der französischen Rundfunkindustrie vor ausländischer Konkurrenz. Schon früh wurden aber auch Gegenstimmen laut, welche die hohe Zeilenzahl aus technischen Gründen ablehnten, bedeutete sie doch, daß die Bandbreite des Übertragungskanals entsprechend größer sein mußte. Auf der CCIR-Versammlung in Atlantic City 1947 hatte man die Bänder I (41 bis $68 \mathrm{MHz}$ ) und III (174 bis $216 \mathrm{MHz}$ ) für den Fernsehrundfunk bestimmt. Eine höhere Zeilenzahl und damit Kanalbreite bedeutete für ein Land, daß ihm weniger Kanäle und damit weniger Fernsehprogramme zur Verfügung standen, da die national zugeteilten Sendefrequenzen begrenzt waren ${ }^{69}$. Benötigte das englische

66 Ibid.S. 208f.

67 AlBert, TudesQ, Histoire de la télévision, S. 71.

68405 Zeilen ergeben ungefähr 160000 Leuchtpunkte auf dem Bildschirm, 625 Zeilen ungefähr 400000 und bei 819 Zeilen sind es über 800000 Leuchtpunkte. Je höher die Anzahl der Leuchtpunkte, um so deutlicher können Helligkeitsunterschiede wiedergegeben und um so schärfer helV dunkel-Konturen sichtbar gemacht werden.

69 RindfLEISCH, Technik im Rundfunk, S. $30 \mathrm{f}$. 
405-Zeilensystem eine Kanalbreite von $5 \mathrm{MHz}$, waren es beim 625 -Zeilensystem schon $8 \mathrm{MHz}$, beim 819-Zeilensystem gar 13,5 MHz. Für Frankreich bedeutete dies, daß mit dem 819-Zeilensystem lediglich ein Programm pro Kanal ausgestrahlt werden konnte ${ }^{70}$.

Da man sich in Europa erst auf der Stockholmer Wellenkonferenz des Jahres 1952 mit der Definition der Kanalbreiten für den europäischen Fernsehfunk befaßte, herrschte bis zu diesem Zeitpunkt ein freier »Zeilenwettbewerb - von jedem Land mit der Hoffnung betrieben, seine Zeilendefinition qua wirtschaftlicher oder politischer Macht als "normgebend " durchsetzen zu können ${ }^{71}$. Wie das Beispiel Frankreich zeigt, gab es auch innerhalb eines Landes konkurrierende Systeme. Um in Frankreich zu einem einheitlichen nationalen Standard zu gelangen, wurde eine »Commission technique« aus Vertretern der Industrie und der staatlichen Rundfunkanstalt (Radiodiffusion Française) gegründet. Bereits im Juni 1947 wurde eine Empfehlung veröffentlicht, die eine Zeilenzahl »d'environ 1000 lignes« vorsah.

Warum setzte sich letztlich das 819-Zeilensystem von Henri de France durch? Es bieten sich mehrere Erklärungsmuster an. Zieht man technische Kriterien zur Bewertung des 819-Zeilensystems im Vergleich zu den anderen Alternativen (1015 und 729) heran, ergab sich 1948 kein zwingendes Argument für das de France-System. Im Gegenteil. Mehrere Argumente sprachen dagegen: zum einen war die Zeilenzahl zu hoch, um bei der beschränkten Kanalbreite eine flächendeckende Versorgung Frankreichs mit 2 oder 3 Programmen gewährleisten zu können. Dies wäre dagegen mit dem 729-Zeilensystem der CFTH möglich gewesen ${ }^{72}$. Zum anderen war die Zeilenzahl im Vergleich zum CdC-System nicht hoch genug, um eine problemlose Übernahme von s/w-Filmmaterial ( $36 \mathrm{~mm}$ Filme) zu gewährleisten ${ }^{73}$. Die Gründe

70 Laut «Bandbreite-Formel« steigt die Bandbreite im Quadrat mit der Zeilenzahl an. Siehe SCHÖNFELDER, Fernsehtechnik im Wandel, S. 8 f.

71 Einen Überblick über die unterschiedlichen Systeme in Europa liefert REICHEL, Auf dem Wege zur europäischen Fernsehnorm. Der politischen und kulturellen Konsequenzen, die dieser Techno-Nationalismus in den Folgejahren für den europäischen Fernsehbetrieb mit sich brachte, waren sich die politischen Entscheidungsträger zu dieser Zeit wohl kaum bewußt. Siehe hierzu Andreas FICKERS, National Barriers for an Imag(e)ined European Community. The Technopolitical Frames of Postwar Television Development in Europe, in: Nothern Lights. Yearbook for Media Studies (2006) S. 7-27.

72 Siehe «Brève histoire des débuts de la télévision française«, 6 maschinegeschriebene Seiten, die der Fernsehingenieur Yves Angel dem Autor im September 1999 zukommen lieB. Angel (geb. 1918), Polytechniker, war ab Oktober 1942 Ingenieur bei der Radiodiffusion Nationale, ab März 1943 Forschungsleiter der Abteilung Fernsehtechnik. Nach dem Krieg war er bis 1947 als leitender Ingenieur in Diensten der RTF, bevor er 1957 als Professor an das CNAM, eine der französischen Eliteschulen wechselte.

73 Siehe Marc Chauvierre, Note sur l'organisation actuelle et l'organisation future de la télévision française, Juni 1949, in: Archives du CHTV/NA, Nachlaß Marc Chauvierre/819-lignes, 5 Seiten, S. 5. 
für die Durchsetzung des 819-Zeilen-Systems müssen daher außerhalb des technischen Bereichs gesucht werden.

\subsection{2. »Toujours $>$ la France seule et plus seule que jamais $"{ }^{74}$}

Schenkt man den offiziellen Darstellungen Glauben, die den am 21. November 1948 getroffenen Entschluß des französischen Staatssekretärs für Information, François Mitterrand, für das 819-Zeilensystem kommentieren, so stand ein Gedanke im Mittelpunkt des französischen Entschlusses: der Schutz der heimischen Industrie vor ausländischer Konkurrenz. Genau dieses Kalkül sollte sich jedoch als Denkfehler herausstellen. Als Ende 1949 die ersten Programme im neuen 819-Zeilenstandard gesendet wurden, war es der niederländische Konzern Philips, der als einziger Anbieter einen funktionsfähigen 819-Zeilenempfänger auf den französischen Markt brachte! Schon bald nach der Normfestlegung regte sich daher bei einem großen Teil der französischen Rundfunkindustrie heftiger Unmut über die Entscheidung.

Am 6. Dezember 1948 erreichte den Generaldirektor der Radiodiffusion Française, Wladimir Porche $e^{75}$, ein Schreiben des Präsidenten und Vizepräsidenten des Syndicat national des industries radioélectriques (SNIR), in dem beide harsche Kritik an der Entscheidung der Regierung übten. Man wundere sich, so die beiden Autoren Damelet und Guillemant, warum die französische Regierung eine Zeilennorm gewählt habe, die so deutlich über den in Europa üblichen Normen liege. Dabei hätten die amerikanischen und britischen Erfahrungen doch gezeigt, da $B$ eine Zeilenzahl um 600 eine absolut befriedigende Bildqualität ergeben würden.

Au surplus, s'il ne nous appartient pas de juger votre point de vue, c'est notre devoir de vous dire qu'il exige à coup sôr de l'économie française, un effort bien supérieur à celui qui eat suffi s'il avait été plus modestement peut-être, mais plus raisonnablement à notre sens, décidé d'adopter pour la France un standard de l'ordre du réseaux en exploitation ou légèrement supérieur à celui-ci. Il nous paraitrait d'autre part regrettable qu'une décision française trop audacieuse risque de compromettre la constitution d'un réseau international de Télévision de l'Ouest Européen dans lequel la culture française devrait normalement trouver des éléments d'expansion extrêmement importants et nous serions heureux de connaitre votre opinion sur cette deuxième question $^{76}$.

74 Zitat aus Chauvierre, La télévision, S. 43.

75 Zur Person Wladimir Porchés siehe die Ausgabe Nr. 11 des Bulletin du Comité d'histoire de la télévision (janvier 1985), in der Porché auf 63 Seiten ein autobiographisches Zeugnis über seine Zeit beim französischen Fernsehen ablegt. Im AnschluB an Porchés Selbstzeugnis ist eine dreiseitige Biographie abgedruckt (S. 66-69).

76 Siehe »lettre de M. Damelet et M. Guillemant à Wladimir Porché, Directeur Général de la Radiodiffusion Française«, Paris, 6 décembre 1948, 5 Seiten, S. 2, in: Archives du CHTV/INA, NachlaB Marc Chauvierre/819-lignes. 
Bedenkt man, daß das SNIR zu diesem Zeitpunkt über 400 Hersteller von Rundfunkempfangsgeräten vertrat, hatte die deutliche Kritik einiges Gewicht. Vor allem der Verweis auf das Problem des europäischen Fernsehnetzes ist von besonderem Interesse. Eine von anderen europäischen Normen unterschiedliche französische Zeilendefinition bedeutete in der Praxis, daß ein Programmaustausch mit diesen Ländern enorm erschwert wurde. Zudem, wie in dem Anschreiben formuliert, wurde dadurch der Export französischer Kultur gehemmt - ein Argument, das in Frankreich stets von staatstragender Bedeutung war $^{77}$.

Die Antwort von Wladimir Porché ließ nicht lange auf sich warten. Erstaunt stellte er fest, daß das SNIR doch an der Diskussion um die Normgebung beteiligt gewesen sei, und man sich im Juni 1947 gar auf eine noch höhere Zeilennorm verständigt habe:

Dois-je encore rappeler que dans les discussions menées au sein de la Commission de Modernisation des Télécommunications - Plan Monnet - et auxquelles votre Syndicat a participé de la façon la plus active, un rapport du SNIR avait été établi qui en matière de télevision appuyait totalement la politique de haute définition [...]. Je suis convaincu que la seule et verritable manière de défendre les intérêts de notre industrie, aussi bien d'émissions que de réceptions, est d'exploiter l'avance technique que nous possédons dans le domaine de la haute définition ${ }^{78}$.

Außerdem, so Porché, komme der aktuelle Zeilenvorschlag doch von Unternehmen, die im SNIR vertreten seien ${ }^{79}$. Auf diese Vorwürfe reagierte Damelet in einem Brief vom 19. Januar 1949, in dem die >wahren`Gründe für die Unzufriedenheit des SNIR mit der Entscheidung für das 819-Zeilensystem zu Tage kamen. Es wird klar, daß es weniger die vorgeschlagene Zeilennorm war, die für Unmut sorgte, sondern die Form des Entscheidungsprozesses. Zu keinem Zeitpunkt, so kritisiert Damelet, habe eine wirkliche Zusammenarbeit zwischen der Industrie und der Radiodiffusion Française und damit der Regierung stattgefunden. "A aucun moment« so Damelet, »n'a existé de commission véritablement responsable d'émettre un avis sur les moyens techniques les plus propres à assurer le développement de la télévision française«. Er schließt seinen Brief mit einer fundamentalen Kritik an der

77 Dieses Argument wird uns auch in der Farbfernsehdebatte häufig begegnen. Zur »mission culturelle de la France« siehe Franz Alting von Gneisenau, Kulturdiplomatie und nationale Stereotypen: nur zum offiziellen Gebrauch?, in: Teresa WaLAS (Hg.): Stereotypen und Nationen, Krakau 1999, S. 305-310; Frank Costigliola, Power and Language: The French Connection, in: Contemporary European History 2 (1993) 1 S. 69-80; Albert SALON, L'action culturelle de la France dans le monde, Paris 1983.

78 »ettre de Wladimir Porché à M. Damelet«, Paris 10 décembre 1948, 4 pages, in: Archives du CHTV INA, NachlaB Marc Chauvierre/819-lignes.

79 Es handelte sich dabei um die Firmen Compagnie des compteurs, Compagnie générale de TSF, Compagnie française Thomson-Houston, Société Radio Industries, Laboratoire générale des télécommunications, Société Sadir-Carpentier. 
Fernsehpolitik, so wie sie seit Ende des Zweiten Weltkrieges in Frankreich betrieben worden sei:

Nous sommes en particulier persuadés que la propagande faite actuellement sur la télévision ne reposera pas sur une base solide tant qu'elle ne sera pas appuyée par l'action des fabricants de récepteurs. Elle risquerait dans sa forme actuelle d'aboutir exactement au résultat opposé au résultat recherché en donnant encore une fois au public l'impression éprouvée par lui depuis 4 ans, que la télévision française est beaucoup plus un sujet de controverse qu'une réalité concrète ${ }^{80}$.

Was hier zur Sprache kommt, weist auf grundlegende Probleme des französischen Nachkriegsfernsehens hin. Zwar konnten in Paris die Versuchssendungen dank der übriggebliebenen deutschen Sendeanlagen rasch wiederaufgenommen werden, Empfangsgeräte waren aber so gut wie keine vorhanden. Bevor die Empfängerindustrie in die schwierige Serienproduktion investierte, mußten sämtliche Normen festgelegt sein. Da dies erst im November 1948 definitiv erfolgte, waren bis zu diesem Zeitpunkt kaum 441-Zeilenempfänger gebaut worden. Wie Damelet zu Recht feststellte, war das Fernsehen in Frankreich bis zu diesem Zeitpunkt tatsächlich eher Gegenstand der Auseinandersetzung um sein zukünftiges Erscheinungsbild denn bildhafte Realität. Da das Fernsehen in den USA und in Großbritannien im Gegensatz zu Frankreich in diesem Zeitraum einen rasanten Aufstieg erlebte, war die Sorge der französischen Hersteller durchaus berechtigt. Durch die Wahl eines von diesen Ländern abweichenden Systems konnten sie schon vor Beginn des Aufbaus einer heimischen Empfängerindustrie ins Abseits geraten ${ }^{81}$. Ein weiteres Problem scheint die mangelnde Kommunikation zwischen Industrie und staatlicher Rundfunkorganisation gewesen zu sein - trotz zentralistisch organisierter Planpolitik. Es kann vermutet werden, daß dies mit der Personalpolitik im staatlichen Rundfunkbetrieb zusammenhing. Wie zentrale Akteure der französischen Rundfunktechnik dieser Epoche berichten, wurden wichtige Entscheidungen auch im Bereich der Technik oftmals aufgrund persönlicher Verbindungen oder Beziehungen getroffen, nicht aufgrund technischwissenschaftlicher Argumente.

80 "Lettre de H. Damelet à Wladimir Porché«, 19. Janvier 1949, 3 pages, in: Archives du CHTV/INA, NachlaB Marc Chauvierre/819-lignes.

81 Pierre Albert weist darauf hin, daß das Erreichen der 1-Million-Empfängergrenze in Frankreich doppelt so lange gedauert hat wie in England oder Deutschland. In Großbritannien war diese Zahl fünf Jahre nach Wiederaufnahme des regelmäßigen Programmbetriebes erreicht (1951). Die 10-Millionen-Grenze wurde 1960 enreicht. In der Bundesrepublik Deutschland, wo der Programmbetrieb erst 1953 startete, war die 1-Million-Grenze 1957, die 10-Millionen-Grenze 1964 erreicht. Setzt man den regelmäBigen Programmbetrieb in Frankreich mit Beginn der 819-Zeilennorm im Jahr 1949 an, dauerte es bis zur ersten Million zehn Jahre, bis zum Erreichen der 10-Millionen-Grenze 20 Jahre! Siehe Pierre ALBERT, Remarques sur la lenteur du développement de la télévision en France, in: Bulletin du Comité d'histoire de la télévision 1 (juillet 1981) S. 17-24. 
Marc Chauvierre ${ }^{82}$, der zu den Pionieren des französischen Fernsehens in den 1930er Jahren zählte, kritisierte scharf das "système de fonctionnaire incompétent et inamovible«, das den staatlichen Rundfunkbetrieb seit 1945 kennzeichne ${ }^{83}$. Auch Yves Angel, von 1945 bis 1955 Technischer Direktor der RDF/RTF ${ }^{84}$, machte in einem Rückblick auf die personellen Verflechtungen zwischen RTF und dem Unternehmen Radio-Industries (RI) aufmerksam, jener Firma, in der Henri de France seit 1933 Chefingenieur und ab 1940 Mitglied des Aufsichtsrates war ${ }^{85}$. Wladimir Porché, der vor Beginn des Zweiten Weltkrieges verantwortlich für die Wortbeiträge im französischen Hörfunk war, trat nach der Okkupation in die Dienste der Radio-Industries ein. Während fünf Jahren war er somit Kollege von Henri de France, bevor

82 Zur Person Marc Chauvierres siehe den Nachruf von Michel Favreau und Michel CARPeNTIER, Souvenir - Marc Chauvierre, pionnier de la télévision nous a quittés, in: Les Cahiers du Comité d'histoire de la télévision 3 (juin 1998) S. 69-71. Wegen seiner Tätigkeit beim Fernsehsender Paris in den Jahren 1943/44 und seiner Suspendierung aus dem Staatsdienst nach der Libération versuchte Chauvierre nach 1945 vergeblich, im staatlichen Nachkriegsfernsehen FuB zu fassen. In einem Brief an den Abgeordneten Max Brusset vom 15. November 1951 empfahl sich Chauvierre als »Retter $\ll$, um das in seinen Augen krisengeschüttelte französische Fernsehen endlich auf den »rechten Weg« zu bringen: »Ce changement de politique, d'ailleurs, ne pourra être effectif que s'il correspond à un changement d'hommes aux échelons les plus élevés des responsabilités. [...] Pour ma part, je suis disposé, comme je vous l'ai déjà dit, à revendiquer la direction de la télévision française, direction à laquelle plus que tout autre mon passé me qualifie. [...] Je crois pouvoir dire que ma nomination serait très bien acceuillie par la majorité des constructeurs français (à l'exception évidemment du constructeur qui a lancé le 819-lignes) et qu'un tel programme serait susceptible de créer le choc psychologique qui seul permettra d'assurer le véritable démarrage de la télévision française«. Lettre de M. Chauvierre à M. Max Brusset, Député de la Charente, Paris, 15 Novembre 1951, 2 maschinegeschriebene Seiten, hier S. 2, in: Archives du CHTV/INA, Nachlaß M. Chauvierre/819-lignes. Die als persönliche Ungerechtigkeit erfahrene Situation von Chauvierre nach 1945 macht ihn zu einem der heftigsten Kritiker der französischen Fernsehpolitik im Bereich der technischen Entscheidungen. Obschon viele seiner Aussagen aus heutiger Perspektive gerechtfertigt scheinen, ist seine offene Kritik am System immer vor diesem privaten Hintergrund zu betrachten. Siehe CHauvierRe, La télévision.

83 Siehe »Note sur l'organisation actuelle et l'organisme futur de la Télévision française «, die Marc Chauvierre im Juli 1949 an Max Brusset, einen Abgeordneten des Département Charente im französischen Parlament richtete. In: Archives du CHTV/INA, Nachlaß Marc Chauvierre/ 819-lignes, 4 Seiten, hier S. 3.

84 Die staatliche Rundfunkorganisation "Radiodiffusion française" (RDF) wird am 4. Februar 1949 per Dekret zur »Radiodiffusion télévision française» (RTF) und am 27. Juni 1964 zur "Organisation de radiodiffusion télévision française» (ORTF). Am 7. August 1974 wird die ORTF aufgelöst. Es entstehen die öffentlich-rechtlichen Fernsehsender TF1, Antenne 2, FR3 sowie der Hörfunksender Radio France. Siehe Bourdon, Haute Fidélité. Am Ende des Buches befindet sich eine ausgiebige Chronologie des staatlichen französischen Fernsehens mit den Themenfeldern "Politique générale et ministres de tutelle«, "réformes et crises«, "les dirigeants» und »les émissions".

85 Siehe Bulletin du Comité d'histoire de la télévision 14 (juin 1986), das Henri de France gewidmet ist und eine stark gekürzte Version seiner unveröffentlichten »Mémoires« sowie zahlreiche Erinnerungen von Weggefährten enthält. 
Informationsminister Gaston Deferre ihn 1946 auf den Posten des Generaldirektors der Radiodiffusion Française berief. Ab diesem Augenblick, so Yves Angel, "le développement de la Radio-Industries devint explosif [...]. La R.I. n'attend plus les commandes. Elle construit. La RTF n'a plus qu'à loger les matériels, les exploiter, et faire des marchés de régularisation pour pouvoir payer des factures ${ }^{86}$. Dennoch, und hier schließt Angel eine deutliche Kritik an der Person Henri de France' als Unternehmer an, sei die RI auf keinen grünen Zweig gekommen:

Malgré sa grande activité, la R.I. fut loin de faire fortune car, au train où allaient les dépenses (campagnes de démonstrations du 819-lignes en France et à l'étranger, 'pêche` d'ingénieurs et de techniciens chez les concurrents, heures supplémentaires, banquets, courtoisies aux hommes politiques...), les difficultés financières réapparurent ${ }^{87}$.

Angels Aussagen werden - zumindest was die enge Verbindung von de France und RTF (Porché) angeht, auch von Ingenieuren der RTF oder Thomson-CSF bestätigt. »Le 819-lignes a été une décision essentiellement politique. Les ingénieurs ont du suivre. [...] Cette décision était en fait largement motivé par le souci de favoriser Radio-Industries et de France«, so Bernhard Hecht, Entwicklungsingenieur der bei der RTF $^{88}$. Ein wenig nüchterner gesteht auch einer der besten Kenner der französischen Rundfunkgeschichte, Jérôme Bourdon: „Dynamique et bien introduit, Henri de France a autant d'entregent politique que d'inventivité ${ }^{89}$. Eine Eigenschaft, die sich - wie zu zeigen sein wird - auch bei der Einführung des Farbfernsehens in Frankreich bezahlt machen sollte. Noch fünf Jahre nach Mitterrands Entscheidung

86 ANGEL, Brève histoire des débuts de la tv française.

87 Angel sieht in dieser Entwicklung gar "symptomatische " Charakteristika von de France's wirtschaftlichen Unternehmungen: »Henri Defrance (j'ortographie le nom selon l'usage de l'époque) fut un sbricoleur de talent<. Avant la guerre, il avait plus au moins dirigé de petites industries (haut-parleurs) que son esprit aventureux a conduites toutes à la faillite. L'une, du nom pompeux de 'Compagnie générale de t.v.র, en difficultés financières vers 1933, fut reprise par la ২Radio-Industries ( $(\mathrm{R}$ I). La caractéristique des krachs qui ont jallonné toute sa vie est qu'à chaque reprise de sa société moribonde par une société en bonne santé, Defrance retrouvait une place de direction qui ne tardait pas à devenir la place du patron, au moins de fait. Et quelques années plus tard, le processus recommencait, la proie grossissant à chaque fois. Il avait le génie de la persuasion pour emporter les affaires «.

88 Siehe Transkription eines Interviews mit Bernhard Hecht vom 4. Juni 1984, in: Archives du CHTV/INA. Im gleichen Bestand befinden sich auch Interviews mit den Ingenieuren Claude Mercier (Technischer Direktor der RTF/ORTF), Jacques Fagot (Technischer Direktor bei Thomson-CSF) und Jean-Jacques Matras (Entwicklungsingenieur der RTF). Auch Jacques Fagot weist auf die engen Kontakte hin, die de France zu den politisch Verantwortlichen pflegte und verweist zudem auf die Parallelität der Entwicklung des 819-Zeilensystems und des Farbfernsehens: "Parallele 819, SECAM? Décisions politiques et nationales. Influence de de France, très introduit. De plus liens familiaux avec Floirat. C'est lui qui a repéché la CFT, parce que Saint-Gobain n'a pas voulu continuer trop longtemps dans cette histoire, après l'adoption du SECAM«. Siehe Interview mit Jacques Fagot vom 25. Mai 1984, S. 6, in: Archives du CHTV/INA.

${ }^{89}$ Bourdon, Haute Fidélité, S. 33. 
verteidigte der französische Abgeordnete Max Brusset die Entscheidung pro 819-Zeilen in seinem 125 Seiten starken »Exposé sommaire sur la situation de la télévision française et ses possibilités d'extension«. Er schreibt:

C'est l'intérêt national bien compris, aussi bien dans la défense du territoire que dans celui de la protection de notre industrie qui a amené le Gouvernement et le Parlement français à choisir cette définition, c'est à dire une technique qui est différente de celles utilisées dans les autres nations industrialisées.[...] Si le Gouvernement et le Parlement n'avaient pas pris la décision de protéger par tous les moyens notre jeune industrie dans ce domaine, le marché français seraient devenu une proie facile pour les grands trusts étrangers contre lesquels nos constructeurs, mis en retard par les destructions de la guerre auraient été incapables de lutter ${ }^{90}$.

$\mathrm{DaB}$ diese fast heroisch anmutende, nationalistisch legitimierte Entscheidung letztlich nicht den erhofften Erfolg hatte, ist für Brusset das Resultat einer „Verschwörung" gegen Frankreich, deren Opfer das französische Volk und ihre Industrie sei:

La guerre du lignage est la cause du handicap français en toute matière: qu'il s'agisse de l'adoption du standard français par d'autres gouvernements, qu'il s'agisse du développement de l'infrastructure sur le sol national, ou encore du développement du nombre des postes récepteurs aux mains des téléspectateurs français. Tout s'est trouvé paralysé par la propagande effrénée qui a été menée contre le standard français et dont notre pays et l'industrie française sont aujourd'hui les premières victimes ${ }^{91}$.

Analysiert man die Gründe für die Einführung des 819-Zeilensystems in Frankreich aus entemotionalisierter historischer Perspektive, so zeigt sich, daß weder im Bereich der Technik noch im Bereich der Industrie zwingende Faktoren für diese Entscheidung vorhanden waren. Zwar war die Entschei-

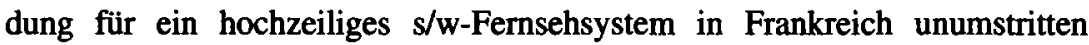
(Bildqualitäts-Argument), jedoch konkurrierten hier mehrere Varianten miteinander, die jeweils ihre eigene technische Legitimation hatten. Das von der Politik verfochtene Protektionismus-Argument (Schutz der heimischen Rundfunkindustrie) erweist sich bei näherem Hinsehen als fadenscheinig (Widerstand des SNIR). Besonders heikel scheint die Entscheidung für das 819-Zeilensystem vor dem Hintergrund des von Politik und RTF gemeinsam propagierten Kulturexport-Argumentes. Gerade die Wahl eines von den anderen europäischen Ländern verschiedenen Standards erschwerte den Programmaustausch enorm. Für die zentralen politischen Akteure (v.a. François Mitterrand) war die in ihren Augen bessere Technik (hochauflösenderes Bild) schon Beweis der kulturellen und wissenschaftlich-technischen Superiorität, der sich die anderen Länder - insofern sie die Zukunft nicht verpassen wollten - schwerlich verwehren konnten (Fortschrittslogik-Argument).

90 Max Brusset, Exposé sommaire sur la situation de la télévision française et ses possibilités d'extension, 125 maschinegeschriebene Seiten, hier S. 37, Paris 1953, in: Archives nationales, Signatur F-41/2303.

91 Ibid. S. 35. 
Zwar lassen sich zahlreiche Argumente aufführen, die die Entscheidung für das 819-Zeilensystem aus der Sicht des politischen Entscheidungsträgers François Mitterrand nachvollziehbar und aus damaliger Perspektive legitim erscheinen lassen, doch zeigt die unmittelbar auf diese Entscheidung folgende Zeitspanne, daß die Entscheidung auf "wenn - dann «-Annahmen fußte, die der wirtschaftlichen und rundfunkpolitischen Realität Frankreichs Ende 1948 nicht entsprachen. Zwei Faktoren müssen herausgestellt werden, will man die Niederlage der von Mitterrand als "guerre du lignage« und »bataille diplomatique « bezeichneten Auseinandersetzung verstehen ${ }^{92}$. Dies sind zum einen die ab 1948 intensivierten Bemühungen um einen europäischen $\mathrm{s} / \mathrm{w}$-Standard im Rahmen der CCIR, zum anderen die mangelnde finanzielle Unterstuitzung des technischen Ausbaus des staatlichen Fernsehrundfunks seitens der französischen Regierung. Auf den ersten Aspekt soll im folgenden näher eingegangen werden, da er den internationalen Kontext, in den die Netzwerktechnologie Fernsehen eingebunden ist, besonders deutlich macht.

\subsubsection{Der institutionelle Verhandlungsrahmen: ITU/CCIR/EBU}

Die Normierung der Zeilenzahl ist in der Logik der »large technological systems « per Definition eine Angelegenheit, die internationalen Charakter hat $^{93}$. Lediglich eine grenzüberschreitende Regelung konnte eine standardisierte oder »kompatible« Lösung bieten. Diese Lösungen herbeizuführen war die Aufgabe des Comité consultatif international de radiotélécommunications (CCIR). Diese Institution, vom ehemaligen Technischen Direktor des NDR, Hans Rindfleisch, als »wissenschaftlich-technisches Gewissen des Weltnachrichtenvereins ${ }^{94}$ bezeichnet, hatte die Aufgabe, die in den verschiedenen nationalen Fernmeldediensten auftretenden technisch-wissenschaftlichen, betrieblichen oder Gebühren betreffenden Fragen - sofern sie eine internationale Regelung erforderten - in Form von Empfehlungen (Recommendations) oder Situationsberichten (Reports) zu klären ${ }^{95}$.

\section{Bourdon, Haute Fidélité, S. $33 \mathrm{f}$.}

93 Siehe Thomas P. Hughes, Large technological systems; sowie Renate MAYNTZ, Thomas P. Hugres, The Development of Large Technological Systems, Frankfurt a.M., New York 1988.

94 Hans RindFleisch, Ton- und Fernsehrundfunk auf der CCIR-Konferenz in Los Angeles, in: Rundfunktechnische Mitteilungen 3 (1959) S. 242.

${ }^{95}$ Zur Geschichte und Funktion der ITU, CCIR und ihrer europäischen Schwesterorganisation, der European Broadcasting Union (EBU), siehe Rüdiger ZeLLER, Die EBU. Union Européenne de Radio-Télévision. European Broadcasting Union. Internationale Rundfunkkooperation im Wandel, Baden-Baden 1999; Zum hundertjährigen Jubiläum der ITU ist der Sammelband International Telecommunication Union (Hg.), From Semaphore to Satellite, Genf 1965 erschienen. Auch die beiden älteren Arbeiten von Codding und Namurois erlauben einen detaillierten Einblick in Funktionsweise und Probleme dieser Institution. Siehe George Arthur Codding, The International Telecommunication Union. An Experiment in International Cooperation, Leiden 
Obwohl sich die CCIR seit ihrer Gründung als Unterorganisation der International Telecommunications Union (ITU, gegründet 1865) im Jahre 1927 sehr erfolgreich um die Normierung des internationalen Funkverkehrs bemüht, besitzt sie keine gesetzgeberische Gewalt zur Durchsetzung ihrer Empfehlungen. Genau wie bei der UNO, deren offizieller Arm die ITU im Bereich Telekommunikation seit 1947 ist, sind die einzelnen Mitgliedsstaaten nicht weisungsgebunden. Eine supranationale Struktur ist somit nicht vorhanden. Eine Tatsache, die vor allem von den technischen Experten immer wieder bemängelt wurde, da »Firmeninteressen und nationales Prestigedenken mit hereinspielen ${ }^{96}$. Vor allem dann, wenn es um Entscheidungen mit »network-markets « im Hintergrund geht ${ }^{97}$. »Hélas «, so bedauerte Marc Chauvierre, »dans ces sortes de discussions, le bon sens est souvent absent; ce sont des questions d'amour-propre ou de nationalisme exacerbé qui dominent les débats où, malheureusement, la technique et l'intérêt général passent au second rang " $"$.

Um die Probleme der Frequenzverwaltung im Bereich des Rundfunks im europäischen Raum besser regeln zu können, wurde bereits 1925 die Union Internationale de Radiodiffusion (UIR) in Genf gegründet. Im Gegensatz zur ITU und ihres Organs für den Funkdienst, der CCIR, geht es bei der UIR nicht um die Zuweisung von Frequenzbereichen und Frequenzbändern für die verschiedenen Funkdienste (Seefunk, Landfunk, Flugfunk, Ton-und Fernsehrundfunk etc.), sondern um die internationale Ordnung der Frequenzzuteilung an die Rundfunksender. Dagegen besteht die primäre Aufgabe der CCIR darin, auf so genannten »Wellenkonferenzen« allgemeinverbindliche Frequenzpläne für den Rundfunk aufzustellen ${ }^{99}$. Die Frequenzzuweisung an einzelne Sender innerhalb des Frequenzbandes bleibt aber den zuständigen Behörden der einzelnen Länder selbst überlassen.

1952; Albert NamuroIs, Problems of Structure and Organization of Broadcasting in the Framework of Radiocommunications, Genf 1964.

96 RindFleisch, Technik im Rundfunk, S. 23.

97 Siehe Stanley Besen, Joseph Farrell, Choosing How to Compete: Strategies and Tactics in Standardization, in: Economic Perspectives 8 (1994) S. 117-131.

98 Chauvierre, La télévision, S. 37.

$99 \mathrm{DaB}$ die Wellenkonferenzen trotz ihrer guten Absichten in politische Gefechte und Machtdemonstrationen münden konnten, zeigt sich deutlich am Beispiel der Kopenhagener Wellenkonferenz des Jahres 1948, die bereits voll im Schatten des Kalten Krieges stand. Siehe Fickers, »Der Transistor«, S. 4-16. Eine wirtschaftshistorische Analyse der Frequenzverteilungsproblematik mittels theoretischer Annahmen der Neuen Institutionenökonomik bietet die Dissertation von Christian Henrich-Franke, Globale Regulierungsproblematiken in historischer Perspektive. Der Fall des Funkfrequenzspektrums 1945-1988, Baden-Baden 2006. Eine allgemeine Einführung zur Rolle internationaler Organisationen in regulativen und koordinativen Standardisierungsprozessen im 19. Jahrhundert siehe Gerold AMBRosius, Regulativer Wettbewerb und koordinative Standardisierung zwischen Staaten. Theoretische Annahmen und historische Beispiele, Stuttgart 2005. 
Angesichts der politischen Bedeutung des Rundfunks überrascht es nicht, daß die Entwicklung der UIR trotz der völkerverständigenden Intention bei ihrer Gründung von nationalen Eitelkeiten und zwischenstaatlichen Interessenkonflikten geprägt war. Wie Zeller feststellt, entstand die UIR »ganz im Zeichen und mit den Idealen des Völkerbundes« und hatte mit den gleichen Autoritätsproblemen wie dieser zu kämpfen ${ }^{100}$. Trotz der noblen Absichten des »Völkerbundes der Broadcaster «, trotz der Existenz bilateraler »Rundfunknichtangriffspakte« oder internationaler »Rundfunkfriedenspakte« tobte in Europa ab 1938 ein »Rundfunk- oder Ätherkrieg«, dem die UIR angesichts ihrer Neutralitätsverpflichtung nichts entgegenzusetzen hatte ${ }^{101}$. Es verwundert daher kaum, daß die Geschichte der UIR auch nach 1945 eng an die politische Großwetterlage gekoppelt blieb. Da das technische MeBzentrum der UIR, welches 1926 als »Ätherpolizei« mit Sitz in Brüssel gegründet worden war, während des Krieges Ziel deutscher Begehrlichkeiten gewesen und u.a. als Abhörstation mißbraucht worden war, traten ab 1941 zahlreiche prominente Mitgliedsstaaten aus der UIR aus ${ }^{102}$. Da die UIR 1945 so faktisch auf einen Torso von »deutschfreundlichen« Staaten reduziert worden war, war sie in den Augen vieler ehemaliger Mitgliedsstaaten diskreditiert.

Obschon das Technische Zentrum seine Arbeit unter der Leitung des belgischen Institut National de Radiodiffusion (INR) und der BBC wiederaufnahm, begann man in Genf mit dem Aufbau eines neuen technischen Zentrums. Folge dieser Entwicklung war ein offener Wettbewerb um die Stellung der UIR im internationalen, vor allem aber europäischen Rundfunk. Als die treibende Kraft in der Beseitigung der UIR zugunsten einer Konkurrenzorganisation erwies sich die Sowjetunion ${ }^{103}$. Im März 1946 forderte sie überraschend die Gründung einer neuen internationalen Rundfunkorganisation, in der alle Satellitenstaaten der UdSSR mit Stimmrecht vertreten sein sollten. Zwar verhinderte die mächtige $B B C$ im Verbund mit den skandinavischen Sendern, daß die zur statuarischen Auflösung der UIR notwendige dreiviertel-Mehrheit zustande kam, doch hinderte dies die Sowjetunion nicht daran, am 28. Juni 1946 zur Gründungsversammlung der Organisation Internationale de Radiodiffusion (OIR) einzuladen ${ }^{104}$. De facto war somit auch die Rund-

100 Vgl. Zeller, Die EBU, S. $22 \mathrm{f}$.

101 Ibid. S. 30 f.

102 Siehe Charles Sherman, Turmoil and Transition in International Broadcasting Organisations 1938-1950, in: Journal of Broadcasting (1971) S. 265-273. Speziell mit Blick auf die deutschfranzösischen Beziehungen siehe Ansbert BaumanN, Zwischen Propaganda und Information: Die Entwicklung der deutsch-französischen Zusammenarbeit in Hörfunk und Fernsehen, in: Revue d'Allemagne 37 (2005) 1 S. 7-28.

103 Léo Wallenborn, From IBU to EBU, in: EBU Review, Part I \& II, Januar und März 1978, S. 25-34 und S. 22-30.

104 Später umbenannt in Organisation Internationale de Radiodiffusion et de Télévision (OIRT) mit Sitz in Prag (ab 1949). 
funklandschaft zweigeteilt: die OIR hatte ihren offiziellen Sitz in Brüssel, die UIR in Genf. Beide beanspruchten bis auf weiteres die technischen Anlagen des Technischen Zentrums in Brüssel für sich.

Der Streit eskalierte, als die ITU 1947 in Atlantic City zu einer Weltfunkkonferenz einlud, deren Ziel die Beseitigung des Wellenchaos war, das der Zweite Weltkrieg hinterlassen hatte. Beide Organisationen, die OIR und die UIR, beanspruchten für sich, als »technische Experten« an dieser Tagung teilnehmen zu können. Beiden wurde schließlich dieser Status verwehrt - sie konnten lediglich als "Beobachter" ohne Stimmrecht teilnehmen. Das gleiche Spiel wiederholte sich auch auf der europäischen Folgekonferenz in Kopenhagen 1948. Da dieser Zustand für beide Parteien unbefriedigend und auf lange Sicht schädlich war, sämtliche Einigungsbemühungen aber gescheitert waren, kam es im Mai 1950 auf Anregung der BBC in Genf zur offiziellen Auflösung der UIR und gleichzeitigen Übertragung ihrer (Rest-)Kompetenzen auf die neugegründete European Broadcasting Union (EBU) ${ }^{105}$.

»Die Gründung der EBU«, so Rüdiger Zeller, »war das Ergebnis der Spaltung Europas in einen westlichen und östlichen Block ${ }^{106}$. Die Parallelität von Rundfunk- und Politikgeschichte verwundert kaum. Im Gegenteil, sie kann mit Konrad Dussel sogar als Charakteristikum der Rundfunkgeschichte gedeutet werden. Der Mythos eines »wertneutralen Raumes«, in dem sich die »technischen Experten« laut eigener Einschätzung im Rahmen ihrer Aktivitäten der CCIR oder EBU zu bewegen glaubten ${ }^{107}$, muß schon aus institutionengeschichtlicher Sicht entzaubert werden. Inwieweit das Selbstverständnis der technischen Akteure innerhalb dieses Handlungsraumes von diesem Mythos geprägt war, wird am Beispiel der Farbfernsehkontroverse genauer zu untersuchen sein.

\subsection{4. »Télévision sans passeport ${ }^{108}$ : 625 Zeilen für den Rest Europas}

In diese für die internationale Rundfunkordnung turbulente Zeit fallen auch die ersten Bemühungen um eine einheitliche europäische Norm im Bereich des Schwarzweißfernsehens. Den Auftakt dieser Überlegungen bildete die CCIR-Konferenz in Genf 1948, wo die 625-Zeilennorm erstmals von mehreren Ländern als möglicher europäischer s/w-Standard vorgeschlagen wur-

105 Wolfgang Degennhardt, Elisabeth Strautz, Auf der Suche nach dem europäischen Programm. Die Eurovision 1954-1970, Baden-Baden 1999, S. 18-26.

106 ZELLER, Die EBU, S. 36.

107 Diese Selbsteinschätzung geht aus den Interviews hervor, die der Autor mit Ingenieuren und Wissenschaftlern Louis Goussot, Yves Angel, Gérard Melchior, Heinwig Lang und Michael Hausdörffer geführt hat.

108 H. MARCEL, Télévision sans passeport! Adaption des télévisieurs aux différents standards, in: Télévision 137 (1963) S. 227-230. 
$\mathrm{de}^{109}$. Erste Studien zu dieser Zeilennorm waren bereits 1946 von sowjetischen Ingenieuren vorgestellt worden, die auf die günstige Umwandlungsfähigkeit der amerikanischen 525 Zeilennorm bei 30 Bildwechseln pro Sekunde auf 625 Zeilen bei 25 Bildwechseln pro Sekunde hingewiesen hat$\operatorname{ten}^{110}$. Ihren stärksten Verbündeten sollte die Sowjetunion mit diesem Vorschlag in der niederländischen Firma Philips finden, deren Motivation für die Propagierung des 625-Zeilensystems aus der Kompatibilität dieses Systems mit dem amerikanischen 525-Zeilensystem herrührt. Als einzige europäische Firma im Bereich der Unterhaltungselektronik, für die der amerikanische Markt schon zu dieser Zeit von großer Bedeutung war, übte Philips auf die europäischen Rundfunkländer einen nicht zu unterschätzenden Druck aus. Dies bekamen auch die Franzosen zu spüren, wie Wladimir Porché zu berichten weiß. So warben die mächtigen Niederländer nicht nur über technische Zeitschriften für das 625-Zeilensystem, sie scheuten sich sogar nicht davor, französische Parlamentarier zu >kaufen und ihre Königin Juliana während eines Besuches beim französischen Präsidenten Vincent Auriol zum Botschafter der Interessen von Philips zu machen ${ }^{111}$. Selbstverständlich wurden diese politischen Initiativen von handfestem Druck auf die französische Rundfunkindustrie begleitet. Der Macht des Eindhovener Unternehmens war man sich in Paris durchaus bewuBt. Genau an jenem 20. November 1948, an dem Mitterrand die französische 819-Zeilennorm per Dekret verkündete, folgte Wladimir Porché in Begleitung seiner technischen Direktoren Général Leschi und Stéphane Mallein einer Einladung von Philips nach Eindhoven. Hatten die Niederländer noch die Hoffnung, die Franzosen für das 625-Zeilensystem gewinnen zu können, war es gerade Porché gewesen, der Mitterrand am Vortag seines Besuches in Eindhoven darum gebeten hatte, das 819-Zeilen-Dekret möglichst als »Reisegepäck« mit nach Eindhoven nehmen zu können, um den Niederländem stolz die französische Entschlossenheit demonstrieren zu können.

Il [F. Mitterrand, A.d.V.] avait comprit que je défendais ce que je nommais le sstandard françaisı, non seulement en artiste, parce que l'image obtenue par Henri de France était plus fine, plus dense que ses concurrents étrangères, mais aussi - peut-être surtout, en économiste patriote: parce que le choix d'un standard qui constituterait, pendant un certain temps, un ,verrou techniquer plus efficace qu'un tarif douanier, permettrait à une industrie électronique française de se

109 Chauvierre, La télévision, S. $36 \mathrm{f}$.

110 Siehe BRUCH, Die Fernseh-Story, S. 131. In einem Interview mit Karl Tetzner am 9.2.1973 behauptet Walter Bruch, er habe die später von den Russen vertretene 625-Zeilennorm $1946 \mathrm{zu}$ sammen mit einem Herm Nowakowski im Oberspreewerk in Berlin entwickelt! Siehe Interview Bruch/Tetzner, in: Privatarchiv Prof. Karl Tetzner, Icking bei München, S. 16.

111 In seinen Erinnerungen beschreibt Wladimir Porché ausführlich seinen Besuch bei Philips in Eindhoven am 20. November 1948, jenem Tag, an dem Mitterrand die französische 819-Zeilennorm per Dekret festlegte. Siehe PoRCHE, Fiat Lux, S. 53-60. 
développer sans licences étrangères, sans tributs, sans l'entrave d'une subordination quelconque $^{112}$.

Dieses Bekenntnis eines "patriotischen Ökonomen« verliert ein wenig an Glanz, wenn man den Ausgang der Geschichte kennt und weiß, daß alleine Philips beim Start des französischen 819-Zeilenfernsehens im Jahre 1950 in der Lage war, entsprechende Empfänger in Serie zu produzieren ${ }^{113}$ !

Trotz der französischen Entscheidungen gingen in einigen Ländern die Bemühungen weiter, zu einer einheitlichen europäischen s/w-Zeilennorm zu gelangen. Die Einführung des 625-Zeilensystems in der Sowjetunion und die wirtschaftlichen Interessen von Philips waren zwei wichtige Argumente für die Propagierung dieser Zeilendefinition. Im Laufe des Jahres 1950 entstand eine breitere Allianz zugunsten dieser Zeilennorm. Auf der CCIR-Konferenz in Stockholm 1948 war eine Studienkommission gebildet worden, die den Auftrag hatte, nach Alternativen für einen europäischen Standard im Schwarzweißfernsehen Ausschau zu halten. Es wurde vereinbart, zu diesem Zweck Studienreisen in die USA, nach Großbritannien, Frankreich und die Niederlande zu unternehmen. Im Juli 1949 traf sich die Studienkommission nach Abschluß der entsprechenden Reisen zu einem ersten Treffen in Zürich, wo man die Studienreisen auswertete und ein ausgiebiges Untersuchungsprogramm definierte, um wissenschaftlich-technische Kriterien für den objektiven Vergleich der unterschiedlichen Systemvarianten zu erarbeiten. Doch schon das Züricher Treffen machte klar, welche Schwierigkeiten diese Aufgabe mit sich bringen würde. In einem zusammenfassenden Bericht machte Stéphane Mallein, technischer Direktor der RTF, klar:

Cette réunion avait mis en lumière les difficultés auxquelles allaient se heurter les tentatives de normalisation, essentiellement en ce qui concerne le nombre de lignes. Ces difficultés provenaient de l'ampleur des intérêts économiques en jeu, chacque pays espérant, en faisant adopter ses normes, conquérir ainsi un large marché pour ses exportations nationales ${ }^{114}$.

Wichtiger als das Zusammentreffen in Zürich war die Tagung der Studienkommission in London vom 8. bis 12. Mai 1950. Hatten sich die Briten in Zürich noch mit den Franzosen zusammengeschlossen, da sie mit ihrem 405-Zeilensystem in einer ähnlich isolierten Position waren, bildete sich in London eine breite Front von 625-Zeilen-Befürwortern. Der Schweizer Postingenieur Dr. Walter Gerber übernahm die Leitung einer Untergruppe der Studienkommission, die sich mit der Festlegung aller mit der 625-Zeilen-

112 Ibid. S. 54.

113 ANGEL, Brève histoire de la télévision française, S. 5.

114 Stéphane MaLlein, Rapport sur les travaux de la $11^{\text {ème }}$ Commission d'études à la VI ${ }^{\text {ème }}$ assemblée plénière du CCIR (Genève 1951), Paris, 24. Oktober 1951, S. 5, in: Archives nationales, 870714/Art. 14, Akten des Ministeriums für Kultur und Kommunikation. Bei diesem fünfzehnseitigen Bericht handelt es sich im Prinzip um einen Rückblick auf die Aktivitäten der Studienkommission 11 von Stockholm 1948 bis Genf 1951. 
norm verbundenen Sekundärnormen (Ton-/Bildfrequenzabstand, Kanalbreite etc.) beschäftigen sollte. "En résumé«, so Mallein in seinem Bericht, »la réunion de Londres s'achevait sur une indiscutable victoire du 625 lignes auquel se ralliaient tous les pays présents de l'Europe continentale à l'exeption de la France ${ }^{115}$.

Auf Initiative der belgischen Delegation wurde in London ein Appell an die französische und britische Regierung verabschiedet, der diese zur Übernahme der 625-Zeilennorm aufforderte. Die Antwort der französischen Regierung lohnt einer näheren Betrachtung. Auf 8 Seiten faßte der Kabinettschef des Informationsministers, Jean d'Avout, die französische Position zusammen. Als Gründe der Entscheidung für das 819-Zeilensystem wurden interessanterweise lediglich technisch-qualitative Kriterien aufgezählt - von der Protektion der heimischen Industrie ist keine Rede mehr. In meisterlichem Diplomatenfranzösisch kehrte d'Avout den Spieß gewissermaßen um:

Cependant, l'industrie française, qui aurait à sa charge l'important volume de fabrication que représente déjà le marché français, encore vierge, n'a certainement nul désir d'imposer à ses voisins l'absorption de sa propre production, mais il va sans dire que dans un esprit de collaboration européenne, elle répondrait aux demandes qui lui seraient adressées ${ }^{116}$.

Aufgrund der technischen Überlegenheit des Systems (Bildqualität) und der großen Investitionen, die der französische Staat bereits getätigt habe, so d'Avout, könne Frankreich daher leider nicht positiv auf den Appell der Studienkommission reagieren. In Wirklichkeit waren in Frankreich zu diesem Zeitpunkt weder von der Privatindustrie noch vom staatlichen Fernsehen hohe finanzielle Investitionen für das 819-Zeilensystem getätigt worden. Weder auf Sender- noch auf Empfängerseite waren 1950 systemtechnisch gesehen Tatsachen geschaffen worden, die ein Umschwenken auf die »europäische« 625-Zeilennorm wesentlich behindert hätten.

Scharfe Kritik an dieser Position wurde von Marc Chauvierre geäußert, der seinen Unmut in einem Brief an den französischen Abgeordneten Max Brusset kundtut, welcher zu dieser Zeit »Président de la Commission de Presse« des französischen Parlaments war. Chauvierre schrieb:

La gestion de la télévision française depuis 1945 s'est donc traduit par un échec total, d'une gravité sans précédent pour l'industrie française. La cause de cet échec est très nette: la politique de la télévision française a été axée sur la perfection technique de la très haute définition, sans tenir compte des problèmes d'exploitation et des problèmes internationaux suscités par l'emploi de la très haute définition; [...] Je proposerai, comme base de mon activité [...]: $1^{\circ}$ Obtenir de toute urgence un accord international européen sur le problème des standards, quitte à faire des

115 Ibid. S. 6.

116 Jean D'Avout: Réponse du gouvernement français à l'appel des sept délégations au CCIR de Londres, Paris, le 10 juillet 1950, 8 Seiten, hier S. 6, in: Archives nationales, 870714/Art. 14. 


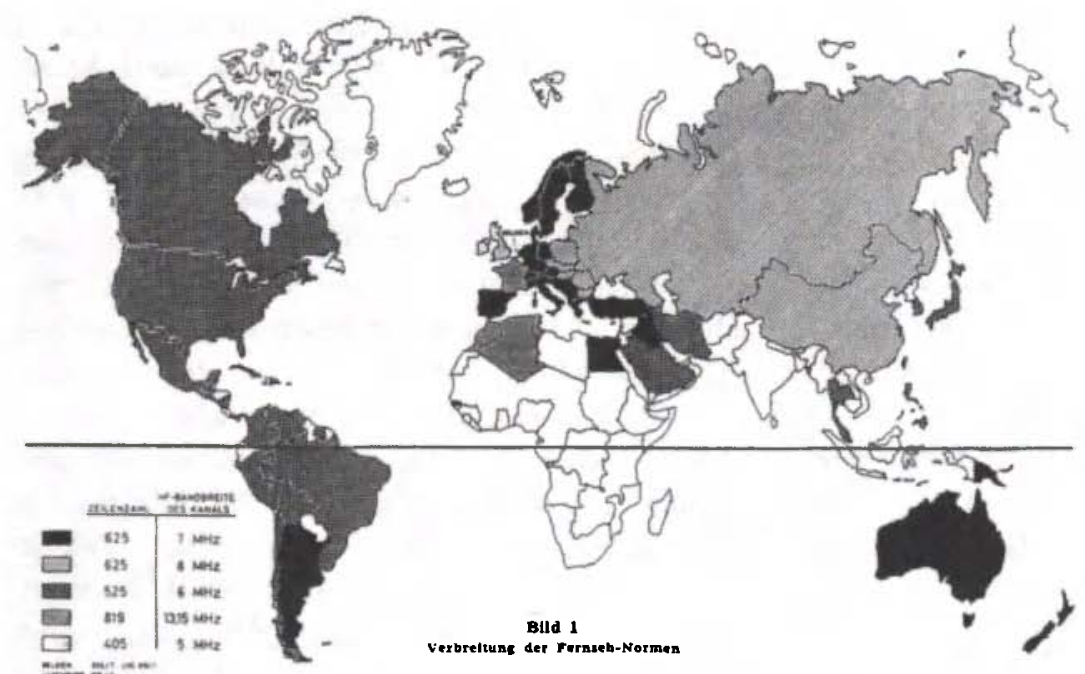

Abb. 3: Verbreitung der Fernsehnormen in Europa, aus: RINDFLEISCH, Der gegenwärtige Ausbau des Fernsehrundfunks im In- und Ausland, S. 220.

concessions importantes pour notre amour-propre, mais indispensable dans le domaine industriel, tant du point de vue français que du point de vue européen ${ }^{117}$.

Doch ein unerwartetes Ereignis sollte der französischen Position für kurze Zeit Rückenwind verschaffen, und zwar die Entscheidung der Federal Communications Commission der USA im Oktober 1950, das von der Columbia Broadcasting Systems (CBS) entwickelte elektro-mechanische Farbfernsehsystem als US-amerikanischen Standard einzuführen. Im Gegensatz zu dem von den CBS-Konkurrenten entwickelten Farbfernsehsystemen der Firmen RCA und Color Television Incorporated (CTI) war das CBS-System nicht kompatibel mit dem bereits vorhandenen 525-Zeilensystem des Schwarzweißfernsehens. Diese für Fernsehfachleute aus aller Welt überraschende Entscheidung wurde von den französischen 819-Zeilen-Protagonisten nicht nur deshalb bejubelt, weil sie das wichtigste Argument der 625-Zeilen-Verfechter aus den Angeln hob, nämlich die Kompatibilität des amerikanischen $525-Z e i l e n / 60 \mathrm{~Hz}$-Systems mit dem europäischen 625 -Zeilen/50Hz-System. Zudem hatten Untersuchungen der RTF-Ingenieure gezeigt, daß das elektromechanische Farbfernsehsystem der CBS im Prinzip kompatibel mit dem französischen 819-Zeilensystem war.

Bereits am 25. Oktober 1950 wurde in der obersten Etage der Radiodiffusion Française ein Strategiepapier entwickelt, um auf diese »unerhoffte Wen-

117 Lettre de M. Chauvierre à M. Max Brusset, Député de la Charente, Paris, 15 Novembre 1951, 2 maschinegeschriebene Seiten, in: Archives du CHTVINA, Nachlaß M. Chauvierre/ 819-lignes. 
de« entsprechend reagieren zu können. In diesem Strategiepapier hieß es: "La décision de la FCC sur la couleur bouleverse la situation internationale en télévision en modifiant radicalement les positions en Europe. Ce bouleversement inattendu par nous et qui fut une des raisons du choix du 819 doit être exploité sans retard par la France qui a une occasion exceptionelle de reprendre la direction du jeu « ${ }^{118}$. Nachdem die möglichen taktischen Verhaltensweisen dargelegt worden waren, endete dieses Strategiepapier mit einem 6-Punkte-Programm, in dem das »déroulement chronologique et psychologique de l'action « festgelegt wurde: Oberstes Ziel war es zu verhindern, daß im Rahmen der CCIR eine Entscheidung pro 625-Zeilen getroffen wurde. Parallel dazu sollten Gespräche mit der Federal Communications Commission und der CBS aufgenommen werden, um beide von den Möglichkeiten der Zusammenarbeit und den Perspektiven für das 819-Zeilensystem zu überzeugen. Bereits im Dezember 1950 trat Stéphane Mallein die angekündigte Reise in die USA an. Sein Reisebericht erlaubt einen interessanten Einblick in die turbulenten amerikanischen Diskussionen, die dort im Anschluß an die Entscheidung der FCC ausbrachen. Mallein betonte die starke politische Dimension, die der Entscheidung der FCC kurz vor den Präsidentschaftswahlen zukam. Die Politik der Wiederbewaffnung im Zuge des Korea-Krieges rückte die Bedeutung der Farbfernsehfrage jedoch weit in den Hintergrund ${ }^{119}$. Auch wenn Mallein abschließend die Chancen einer produktiven Zusammenarbeit mit den Amerikanern im Bereich des 819-Zeilensystems als ziemlich gering bewertete, sah er in der nachlassenden Unterstützung für das 625-Zeilensystem eine große Chance für das französische System - dies auch in bezug auf das noch in weiter Ferne liegende Farbfernsehen in Europa: "Il apparait cependant que la récente décision de la FCC vient appuyer d'une facon aussi éclatante qu'inattendue la thèse que nous n'avons cessé de défendre, à savoir que l'intégration future de la couleur ne pourrait s'opérer facilement que si

118 Stéphane Mallein, Jean D'ARCY, Étude des premières conséquences que la France doit tirer de la décision américaine sur la télévision en couleurs, (très confidentiel), Paris, 25 octobre 1950, 4 maschinegeschriebene Seiten, hier S. 1, in: Archives nationales, 870714/Art. 14.

119 „Un demier aspect, tout à fait inattendu celui-là, est 1'aspect politique. La décision de la FCC a été prise en effet peut avant les élections, et du coup les opposants se sont tournés vers le Parti Républicain qui n'a pas manqué d'accuser le Gouvernement Démocrate d'organiser une véritable dictature étatique ‘ en matière de télévision. En contre partie, bien entendu, les démocrates se sont vus dans l'obligation de soutenir la décision de la FCC ce qui complique encore une situation déjà passablement embrouillée. [...] A vrai dire, avec la nouvelle politique de réarmement qui entraîne déjà la raréfaction des pièces détachées et peut-être bientôt l'interdiction de vente des récepteurs de télévision, la querelle de la couleur prend une allure plutôt byzantine et passe à l'arrière-plan. On en reparlera lorsque l'atmosphère internationale sera éclaircie, c'està-dire dans un délai totalement indéterminé.« Stéphane MaLLein, Rapport sur la mission effectuée à New York et Washington du 14 au 23 décembre 1950, Paris, 31 janvier 1951, 10 maschinegeschriebene Seiten, hier S. 4f, in: Archives nationales, 870714/Art. 14. 
l'on part initialement d'un système blanc et noir à fréquence de ligne élevée et à large bande « ${ }^{120}$.

Als sich die Studienkommission XI (Fernsehen) des CCIR und die von Dr. Gerber geleitete Unterstudiengruppe im Mai 1951 in Genf wiedertrafen, hatten sich die wirtschaftspolitischen und rundfunkpolitischen Kontexte im Vergleich zur vorangegangenen Tagung in London deutlich gewandelt. Im s/w-Normenstreit war ein technischer Gesichtspunkt aufgetaucht, der bislang im europäischen Diskurs nur ganz am Rande thematisiert worden war: die zukünftigen Normen und Standards des Farbfernsehens. Obwohl das französische Kalkül aufging, in Genf eine einheitliche Empfehlung seitens der CCIR zu vereiteln, konnte Frankreich außer Belgien und Luxemburg keine weiteren Anhänger für sein 819-Zeilensystem gewinnen. Auf der Vollversammlung der CCIR in Stockholm 1952, die im wesentlichen der Korrektur des 1948 in Kopenhagen festgelegten Wellenplans gewidmet war, wurde schließlich von den meisten europäischen Ländern die als »Gerber-Norm« bezeichnete 625-Zeilennorm als s/w-Standard übernommen.

De facto herrschten in Europa 1952 also drei verschiedene Zeilennormen: In Großbritannien blieb man vorerst bei 405 Zeilen, in Frankreich, Luxemburg, dem französischsprachigen Teil Belgiens und dem Vatikanstaat setzte sich das 819-Zeilensystem durch. In allen anderen europäischen Ländern (inklusive Sowjetunion) verständigte man sich auf das 625-Zeilensystem. Allerdings konnten auch bei gleicher Zeilenzahl noch Unterschiede bestehen. So hatten beispielsweise die sogenannten Ostblockländer einen von den westeuropäischen 625-Zeilenländern unterschiedlichen Ton-/Bildfrequenzabstand, so daß man zwar diesseits und jenseits des »Eisernen Vorhangs « s/w-Bilder empfangen konnte, aber entweder den Ton hören und kein Bild sehen konnte oder Bilder ohne Ton sah. Durch entsprechende Zusatzgeräte (Transcoder) ließ sich dieses Handicap aber lösen, vorausgesetzt man konnte sich die entsprechenden Teile besorgen und leisten.

Besonders absurd war die Situation in Belgien, wo sowohl der 819- als auch der 625-Zeilenstandard eingeführt wurde. Für die Studio- und Sendertechnik bedeutete dies einen enormen Mehraufwand, da alle Programme in zwei Normen aufgezeichnet und ausgestrahlt werden mußten. Für jene Belgier, die flämisches und französischsprachiges Fernsehen empfangen wollten, bedeutete dies, daß sie entsprechend teurere Mehrnormenempfänger kaufen mußten. Da Frankreich zudem einen von Belgien unterschiedlichen Ton- Bildfrequenzabstand wählte, existierten in Belgien beim Schwarzweißfernsehen 4 verschiedene Standards: $2 \times 819$ (französisch und wallonisch) und $2 \times$ 625 (niederländisch und flämisch). »Les téléviseurs belges acquirent à juste titre la réputation d'être les plus compliqués«, so der Rundfunkingenieur $\mathrm{H}$. 
d'Haese in einem Überblick über die belgische Rundfunkindustrie im Jahre $1963^{121}$.

Bedeutete das Scheitern der Bemühungen eines einheitlichen s/w-Standards in Europa besonders für kleine, mehrsprachige Länder wie Belgien, Luxemburg oder die Schweiz einen enormen technischen und damit finanziellen Mehraufwand ${ }^{122}$, stellte diese Normenvielfalt auch für den internationalen Programmaustausch ein großes Problem dar.

\subsubsection{Eurovision - »ein Stück auf dem Weg $z$ einem gemeinsamen Europa " ${ }^{123}$ ?}

Die Idee des internationalen Programmaustauschs beflügelte schon früh die Phantasie vieler Bildungspolitiker und Intellektueller ${ }^{124}$. So verwundert es kaum, daß diese Überlegungen nach der geistig-moralischen Krise Europas nach dem Zweiten Weltkrieg erneut aufflackerten - auf die Verwandtschaft von Völkerbund und UIR wurde ja bereits hingewiesen. Im Rahmen der Nachfolgeinstitution des Völkerbundes, der United Nations Organisation (UNO), begannen bereits 1947 die Diskussionen über die Möglichkeiten eines internationalen Programmaustausches als Mittel der kulturellen Völkerverständigung.

Motor dieser Bemühungen war der Schweizer Marcel Bezençon, der den Verwaltungsratsmitgliedern der neugegründeten EBU bereits auf ihrer zweiten Sitzung im Mai 1950 einen Plan zum Fernsehprogrammaustausch vorleg$\mathrm{te}^{125}$. Allerdings zeigten sich die Verantwortlichen der EBU recht zurückhaltend, verstanden sie sich doch als »technische Experten «, die kaum Bezug zur inhaltlichen Gestaltung des Fernsehrundfunks hatten. Nicht wenige hatten die Vermutung, daß auch die Programmleute von den angenehmen Nebeneffekten der internationalen Rundfunkorganisation profitieren wollten - das heißt von der Möglichkeit des vielen Reisens. »I suspected«, so der britische EBU-Vertreter Sir Ian Jacob, » - with some justification - that some of the

121 H. D'HAESE, Un récepteur TV pentastandard à automatisme intégral, in: Télévision 135 (1963) S. 179-182, hier S. 179.

122 Vergessen sollte man hier auch nicht die oft mehrsprachigen europäischen Grenzregionen. Auch Frankreich bekam diese »Isolation« sehr bald zu spüren: "Les effets de notre isolement se sont vite fait sentir; très rapidement, il a fallu créer des récepteurs capables de recevoir les deux standards pour les régions frontalières, proches de l'Allemagne et de la Belgique; de tels récepteurs étaient forcément couteux, compliqués et fragiles«. ChaUvierRE, La télévision, S. 38.

${ }^{123}$ Zitat aus Hans RindFLeisch, Die UER in ihrer Bedeutung fuir die europäische Fernsehtechnik, in: Rundfunktechnische Mitteilungen 8 (1964) 6 S. 315.

124 Zur EBU und den Eurovisionsaktivitäten siehe als Standardwerk die Dissertation von DEGENHARDT, Siegen 2002.

125 Siehe Degenhardt, Strautz, Auf der Suche nach dem europäischen Programm, S. 29. 
Abb. 4: Die erste transnationale Live-Fernsehübertragung zwischen Frankreich und Großbritannien war technisch nur durch eine Transcodierung des französischen 819-Zeilenbildes in ein britisches 405-Zeilenbild möglich. Da 1950 noch keine elektronischen Transcoder zur Verfiigung standen, geschah dies auf recht primitive Weise dadurch, das an der Schnittstelle der Verbindung des französischen mit dem britischen Übertragungsweges in Cassel $(F)$ eine 405-Zeilen Fernsehkamera der BBC vor einen französischen 819-Zeilen-Empfänger plaziert wurde. Aus: BBC Audiovisual Archives,

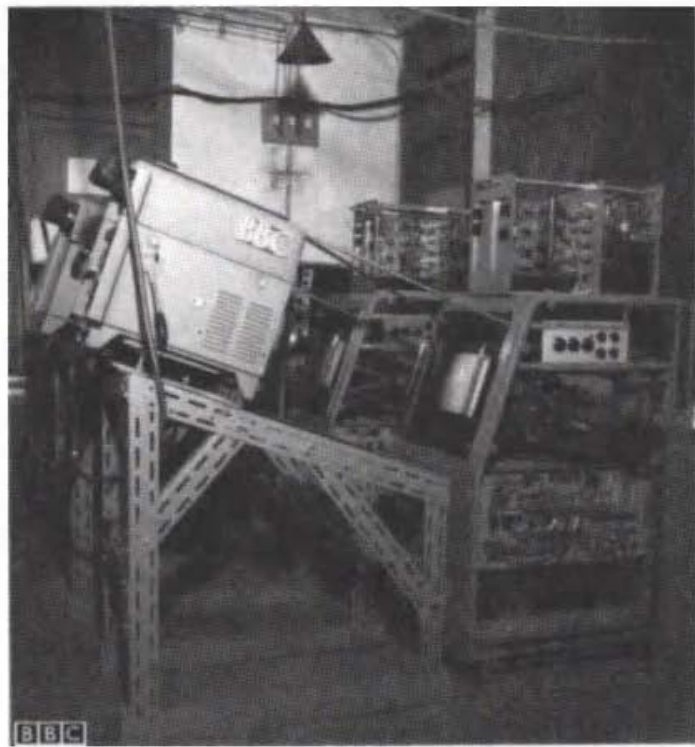
Brentford.

delegates, they wanted another annual jolly ${ }^{126}{ }^{12}$. Auch der technische Direktor des NDR, Hans Rindfleisch, fragte in einem Zeitschriftenartikel ironisch: »Ist sie [die EBU, A.d.V.] etwa eine Einrichtung, um einigen reiselustigen Rundfunkleuten ein paarmal im Jahr eine Art von Zusatzurlaub an einem schönen Fleck irgendwo in Europa zu ermöglichen? «"

Wie so häufig in der Geschichte des Rundfunks schaffte auch hier die technische Weiterentwicklung neue Tatsachen für die Programmplaner. Während man im Verwaltungsrat der EBU die juristischen Probleme des Programmaustauschs diskutierte, fand am 27. August 1950 das sogenannte $\gg \mathrm{Ca}-$ lais-Experiment « statt, bei dem es britischen und französischen Fernsehfachleuten gelang, die erste stehende Fernsehverbindung über Funk zwischen beiden Ländern zu realisieren. Obwohl diese erste transnationale Fernsehverbindung in Europa als medienhistorische "entente cordiale « gefeiert wurde, handelte es sich nicht im eigentlichen Sinne um einen Programmaustausch, da die BBC ihr eigenes Fernsehteam mit britischer 405-Zeilentechnik nach Calais sandte. Ein Austausch auf der Basis realer Normwandlung hatte also nicht stattgefunden. Die ersten Normwandler wurden erst im Laufe des Jahres 1952 entwickelt. Sie waren die Voraussetzung für die erste wirkliche

126 Ibid. S. 27.

127 RINDFLEISCH, Die UER in ihrer Bedeutung für die europäische Fernsehtechnik, S. 310. 
Live-Übertragung eines Ereignisses in verschiedene europäische Länder: der Krönung Elisabeth II. von England am 2. Juni $1953^{128}$.

$\mathrm{Zu}$ Recht wird von zahlreichen Autoren darauf verwiesen, daß dieses Ereignis den eigentlichen Beginn des internationalen Programmaustausches, der Eurovision und damit auch des kommerziellen Fernsehens in Europa bil$\operatorname{dete}^{129}$. Obwohl die EBU keinerlei organisatorische Verantwortung im Rahmen der Übertragung trug, gab die erfolgreiche bilaterale Zusammenarbeit den britischen, französischen, westdeutschen, niederländischen, dänischen, belgischen und italienischen Rundfunkorganisationen den entscheidenden AnstoB zur institutionellen Verankerung des Programmaustauschs in den Statuten der EBU. Auf der Verwaltungsratssitzung der EBU in Monte Carlo im November 1953 wurde auf Drängen Bezançons die »Fernsehkommission" der EBU gegründet ${ }^{130}$.

Die Übertragung der Krönungsfeierlichkeiten war aber mehr als der technische Beweis der Möglichkeit internationalen Programmaustausches: Dieses Ereignis verhalf dem Medium Fernsehen als solchem zum Durchbruch und löste es aus dem Schatten des Radios, das bis zu diesem Zeitpunkt die Funktion des »Leitmediums « im massenmedialen Ensemble innehatte ${ }^{131}$. War das Fernsehen bis zu diesem Zeitpunkt in ähnliche kulturelle Legitimationsprobleme eingebunden wie das Radio zu seinen Anfangszeiten, verhalf die Faszination der Live-Bilder dem Fernsehen zur gesellschaftlichen Akzeptanz ${ }^{132}$. Die Krönungszeremonie der britischen Königin am heimischen Bildschirm

128 Zur technisch-organisatorischen Vorgeschichte dieser Übertragung siehe DegENHARDT, Strautz, Auf der Suche nach dem europäischen Programm, S. 33-38, sowie F. Williams, M. J. L. Pulling, Engineering Arrangements for Broadcasting on Sound and Television for the Coronation of her Majesty Queen Elizabeth II, in: EBU-Bulletin 20 (1953) S. 391.

$129 »$ En tout cas, on peut dire que la retransmission du couronnement de la Reine d'Angleterre a été le signal de départ de la télévision commerciale (et non plus expérimentale) et de l'Eurovision $\ll$. Chauvierre, La télévision, S. 41.

130 Siehe Degenhardt, Strautz, Auf der Suche nach dem europäischen Programm, S. 40.

131 Zum Konzept der »massenmedialen Ensembles« siehe SchiLDT, Das Jahrhundert der Massenmedien. Zur Rolle der »Leitmedien« in der medialen Öffentlichkeit siehe WEISBROD, Medien als symbolische Form der Massengesellschaft.

$132 \mathrm{Zu}$ den Durchsetzungsproblemen des »Radios» und den raum-zeitlichen Herausforderungen dieses neuen Mediums für die Menschen in den zwanziger Jahren siehe die ausgezeichnete Arbeit von LENK, Die Erscheinung des Rundfunks. Zu den Problemen der gesellschaftlichen Akzeptanz des Fernsehens in Frankreich heißt es bei Henri de France: „Ceux que l'on appelle ll'elite، française était dans sa grande majorité contre la télévision, perturbatrice de la vie sociale et surtout familiale. Combien de fois ai-je entendu que l'influence de la télévision sur les enfants est désastreuse, qu'elle donne le mauvais exemple, qu'ils ne font plus leurs devoirs, etc... Cette attitude de rejet devant la nouveauté est typiquement française«. Henri dE FranCE, Mémoires, in: Bulletin du Comité d'histoire de la télévision 14 (juin 1986) S. 11. Siehe hierzu auch STEINMaURER, Tele-Visionen sowie SPIGel, Make Room For TV und John EldIs, Seeing Things. 


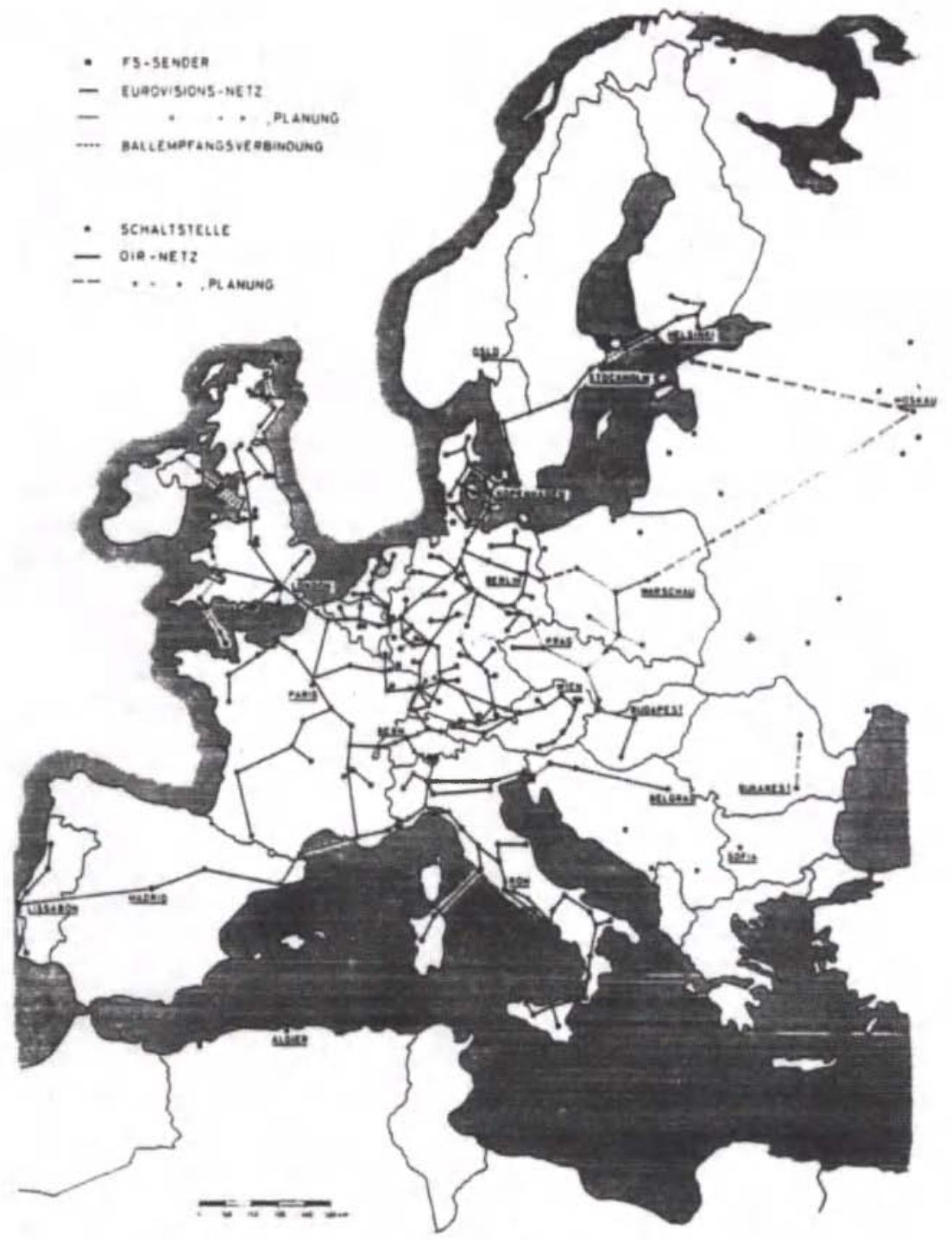

Abb. 5: Kartographische Darstellung des EBU-Eurovision-Netzwerkes im Dezember 1954, aus: $B B C$ Audiovisual Archives, Brentford.

verfolgen zu können war jene Form »privilegierten Sehens«, die zum zentralen Bestandteil der »medialen Identität « des Fernsehens wurde ${ }^{133}$.

133 Siehe Elsner, Müller, Spangenderg, Der lange Weg eines schnellen Mediums, sowie Bernold, Fernsehen ist gestern. Eine konzise Definition der medialen Identität des Fernsehens bietet Jostein GrIPSRUD, Television, Broadcasting, Flow. Key Metaphors in TV Theory, in: Chri- 
Das Fernsehen, das noch immer von vielen als technische Spielerei ohne Zukunft angesehen wurde, hatte seine Fähigkeit bewiesen, Bilder lebendig über Ländergrenzen hinweg zu transportieren. Der Aufwand dafür war enorm, das war unbestreitbar, aber der propagandistische Effekt dieser Pionierleistung für das Fernsehen war mit Geld kaum aufzuwiegen ${ }^{134}$.

Auch die mentalitätsgeschichtliche Dimension der Eurovision sollte nicht unterschätzt werden. Zwar ist es schwierig, ihre identitätsstiftende Wirkung rezeptionsgeschichtlich nachzuweisen, so deutet das Selbstverständnis vieler technischer Akteure, welche diesen Programmaustausch erst ermöglichten, eindeutig in diese Richtung. Besonders deutliche Selbstzeugnisse dieser Art verfaBte der ehemalige Technische Direktor des NDR und langjährige Vertreter Westdeutschlands in zahllosen technischen Kommission der CCIR und EBU, Hans Rindfleisch. In einem Aufsatz aus dem Jahre 1959 mit dem Titel »Der gegenwärtige Ausbau des Fernsehrundfunks im In- und Ausland« heißt es:

Ein wenig ist sowohl die Verschiedenheit der Normen als auch ihre gleichzeitige weltweite Verbreitung ein Spiegelbild des gegenwärtigen Allgemeinzustandes der Menschheit, der durch seine Zerrissenheit und zugleich durch die weltweite Ausdehnung politisch-wirtschaftlicher Systeme gekennzeichnet ist. Auf dem Gebiet des Fernsehens wiegen glücklicherweise die Unterschiede der Normen nicht so schwer wie im politischen Bereich. Für den Austausch von Fernsehsendungen über die Grenzen hinweg ist die Bereitschaft zum Austausch allein entscheidend. Die Geschichte der Eurovision hat bewiesen, daB Unterschiede in der Norm, die in Westeuropa in denkbar bunter Fülle vorhanden sind, auf die Dauer kein ernsthaftes Hindernis für einen ProgrammAustausch darstellen ${ }^{135}$.

An anderer Stelle führt Rindfleisch aus:

Die Mitarbeit in internationalen Organisationen ist oft mühsam und langwierig. Man muB vor allem lernen, die Wünsche und Bedürfnisse anderer Nationen zu verstehen und zugleich die eigenen Wünsche und Bedürfnisse den anderen verständlich zu machen. Aber gerade dies ist der Weg zu einer wirklichen Gemeinsamkeit, und so ist die Mitarbeit in dem Kreis, von dem ich ihnen berichtete, zugleich ein Stück auf dem Weg zu einem gemeinsamen Europa ${ }^{136}$.

Auch wenn es ein wenig pathetisch klingen mag, so kann man davon ausgehen, daß bei einigen dieser Akteure tatsächlich die Motivation zu »wahrhafter Kommunikation« im Jaspersschen Sinne einen Antrieb für ihre Arbeit darstellte ${ }^{137}$. Auf die Rolle der Ingenieure und ihres Selbstverständnisses

stine Geraghty, David Lusted (Hg.), The Television Studies Book, London u.a. 1998, S. 17-32.

134 Degenhardt, Strautz, Auf der Suche nach dem europäischen Programm, S. 38.

135 RindFleisCH, Der gegenwärtige Ausbau des Fernsehrundfunks im In- und Ausland, S. 220.

136 RINDFLEISCH, Die UER in ihrer Bedeutung für die europäische Fernsehtechnik, S. 315.

137 In einem kurzen Vortrag mit dem Titel »Vom europäischen Geist «, den Karl Jaspers im September 1946 bei den »Rencontres Internationales de Genève« hielt, heißt es: »Was im ganzen geschieht, wird ermöglicht durch das, was jeder einzelne tut. Der einzelne muß von sich fordern: sich auf den Standpunkt eines jeden anderen versetzen, Wahrheit in Kommunkation an den Tag bringen, sein Herz nicht verhärten, sondern offen bleiben im Hören, in der Bereitschaft zu tätiger Hilfe und zum Revidieren eigener Ansichten. Die Möglichkeiten der Kommunkation werden zur 
wird im Kapitel »nationale Technikstile und Technikkulturen« noch ausführlich eingegangen. An dieser Stelle mag der Hinweis auf die interessante Verbindung beruflicher Identifizierung technischer Akteure mit den gesellschaftspolitischen Kontexten genügen. Neben dieser psychohistorischen Komponente lassen sich aber weitere Informationen aus diesen Aussagen Rindfleischs gewinnen, so beispielsweise der »nichts-ist-unmöglich «-Glaube an die nahezu unbegrenzten Möglichkeiten der Technik. So bekundet auch Marc Chauvierre in Bezug auf das Problem der unterschiedlichen Zeilendefinitionen im Schwarzweißfernsehen: »Évidemment, il n'y a pas de problèmes que les techniciens ne sachent résoudre. Et ceux-ci ont créé les convertisseurs de standard « ${ }^{138}$. So einfach ist das also. Max Brusset, Abgeordneter im französischen Parlament, präzisierte 1953 in seinem »Exposé sommaire sur la situation de la télévision française et ses possibilités d'extension«:

Les détracteurs du standard français ont conclu bien trop rapidement que la France, dans le jeu des échanges internationaux des programmes se trouverait isolée du fait de son standard. Il n'est possible d'avancer cette objection depuis juillet 1952. Grâce à l'ingéniosité des inventeurs français, un convertisseur de définitions a été mis au point ${ }^{139}$.

Warum, so fragt man sich, hat man sich dann derart über die verschiedenen Varianten und deren Qualitäten gestritten? Auch auf diese Frage weiß Marc Chauvierre eine Antwort: »Le vrai problème ne réside pas dans la compatibilité des systèmes ou de l'importance du >parcı en service. Les questions de personnalité (les techniciens ne sont pas des saints), de prestige pour les firmes et de nationalisme quand il s'agit de normes à l'échelle mondiale, jouent hélas beaucoup plus que tous les arguments ${ }^{140}$.

Diese Stellungnahme eines Menschen, der sich selbst als begeisterter Fernsehtechniker bezeichnet, weist den Weg in Richtung zwischenmenschlicher, sozialer und wirtschaftspolitischer Aspekte, die bei der anstehenden Analyse der Farbfernsehauseinandersetzung verstärkt berücksichtigt werden müssen. Reichen die innertechnischen Argumente für den Beweis des »besten Systems « nicht aus, so die These, werden außertechnische Faktoren bei der Entscheidungsfindung um so bedeutender. Im Falle von Netzwerktechnologien können dies staatliche, privatwirtschaftliche oder privatrechtliche Faktoren sein. Handelt es sich zudem wie im Falle der Fernsehstandards um die Aushandlung technischer Normen, kommen Faktoren wie die der Persönlichkeit der verhandelnden Akteure, soziale und institutionelle Handlungsspielräume sowie kulturelle Verhaltensmuster hinzu, die den Ausgang derartiger Verhandlungsprozesse unvorhersehbar machen. Standardisierungsprozesse bieten

Grundfrage des zu sich selbst kommenden Menschen.« Karl JASPERs, Vom europäischen Geist, München 1946, S. $24 f$.

138 Chauvierre, La télévision, S. 41.

139 Brusset, Exposé sommaire, S. 39.

140 ChauvierRe, La télévision, S. 203. 
sich aus diesem Grunde besonders dazu an, das teleologische Fortschrittsmodell der Technikentwicklung zu hinterfragen, beziehungsweise einen Beitrag zu seiner theoretischen Demontage zu leisten.

\subsubsection{Das Scheitern der Bemühungen um einen europäischen s/w-Fernsehstandard - zusammenfassende Betrachtung}

Versucht man die Gründe für das Scheitern einer europäischen s/w-Fernsehnorm für Europa zusammenzufassen, zeichnet sich ein breites Spektrum nachvollziehbarer Gründe für dieses Scheitern ab. Auch wenn der französischen Diskussion um das 819-Zeilen-System im Vorfeld aus begründetem Anlaß besondere Aufmerksamkeit geschenkt wurde, bietet sich folgendes Panorama von Erklärungsmustern an:

1) Fernsehen war zu dem Zeitpunkt, in dem die Entscheidung für einen einheitlichen europäischen Standard fallen mußte (1946-1952), in Europa kaum praktische Realität. Das Fernsehen war in seiner grundlegenden technischen Entwicklung kaum aus dem experimentellen Stadium herausgetreten. Der Elan, der die Fernsehforschung ab Mitte der dreißiger Jahre ausgezeichnet hatte, war durch den Zweiten Weltkrieg gebrochen worden. Zwar waren während des Krieges wichtige Forschungen in originär fernsehtechnischen Bereichen geleistet worden (Elektronenröhre, Zentimeterwellen, Richtfunk etc.), doch mußten diese unterschiedlichen Stränge nach 1945 erst wieder auf das zivile Anwendungsziel Fernsehen zusammengeführt werden. In Deutschland, einem der führenden Fernsehländer vor Ausbruch des Krieges, war nach 1945 jegliche Arbeit im fernsehtechnischen Bereich verboten. Es verwundert daher nicht, daß Deutschland in der Diskussion um die s/w-Zeilennorm kaum eine Rolle gespielt hat. Wie gesehen, wichen führende deutsche Fernsehtechniker nach Frankreich aus, um weiterhin im Bereich der Fernsehtechnik arbeiten zu können.

2) In Europa entfaltete das Fernsehen als neues Medium nicht jene visionäre Potenz, die ihm in den USA zugeschrieben wurde. Europa blieb vorerst "radiotreu«. Die Ablösung des Leitmediums Radio durch das Fernsehen im massenmedialen Ensemble setzte erst Ende der 1950er Jahre ein. Zudem hatten die totalitären Erfahrungen mehrerer europäischer Staaten die Möglichkeiten eines Mißbrauchs des Rundfunks gezeigt, so daß führende Kulturkritiker dem neuen elektronischen Bildmedium sehr skeptisch gegenüberstanden. Die suggestive Kraft und damit potentiell manipulative Wirkung, die dem bewegten Bild bis heute zugeschrieben wird, erschwerte dem Fernsehen die gesellschaftliche Akzeptanz, zumindest bei der kulturellen Elite. Es bedurfte der affektiven Aufladung des Mediums, um es in das allgemeine Bewußtsein zu bringen. »Media events« wie die Krönung Elisabeth II im Jahre 1953 wirkten hier bahnbrechend. In der Folgezeit waren es vor allem sportliche 
Großveranstaltungen, welche diese Funktion übernahmen (Fußballweltmeisterschaft in Bern 1954; Olympische Sommerspiele in Rom 1960).

3) Da die nationalen Rundfunkorganisationen nach 1945 mehr oder minder damit beschäftigt waren, den Status quo der Vorkriegszeit wieder herzustellen, blieben Ideen wie die einer zukünftigen europäischen Zusammenarbeit Ausnahmeerscheinungen. Ein Problembewußtsein dafür, was unterschiedliche s/w-Normen für den internationalen Programmaustausch bedeuteten, war nicht vorhanden. Der Aufbau einer funktionierenden Rundfunkversorgung blieb im wesentlichen eine nationalstaatliche Angelegenheit.

4) Zusätzlich färbte die politische Großwetterlage Europas nach 1945 auf die internationalen Rundfunkorganisationen ab. Die völkerverbindenden Intentionen der Union Internationale de Radiodiffusion (UIR) scheiterten an den politischen Realitäten des Kalten Krieges. Die Spaltung der UIR in eine "östliche (Organisation Internationale de Radiodiffusion, OIR) und "westliche« (Union Européenne de Radiodiffusion, UER) Organisation erschwerte die internationale Verständigung im Bereich der Rundfunkplanung.

5) Wie am Beispiel Frankreichs näher ausgeführt wurde, waren es nicht zuletzt wirtschaftspolitische Entscheidungen, die den nationalen Diskurs dominierten. Der Schutz der nationalen Rundfunkindustrie spielte auch in Großbritannien oder den USA eine bedeutende Rolle. Der rasche Aufbau einer nationalen Fernsehempfängerindustrie hatte das Ziel, andere Länder von den Vorteilen des eigenen Systems bzw. der Leistungsfähigkeit der eigenen Industrie zu überzeugen. Wirtschaftstheoretisch formuliert: Man erhoffte sich »first-mover«-Vorteile und damit erhöhte Exportchancen. Da der Fernsehmarkt ein »network market« ist, kommt es entscheidend darauf an, den eigenen Standard international durchzusetzen. In Frankreich kamen sicherlich auch protektionistische Interessen hinzu, um die noch schwache eigene Rundfunkindustrie vor der mächtigen amerikanischen und englischen Konkurrenz zu schützen.

6) Die relative Zurückhaltung, welche die Position der USA in der europäischen Auseinandersetzung um die s/w-Fernsehnormen auszeichnete, hatte zwei Gründe. Zum einen stieg das Schwarzweißfernsehen in den USA nach dem Zweiten Weltkrieg rasant zum massenmedialen Leitmedium auf. Der daraus resultierende Boom der Fernsehbranche verschaffte den USA einen erheblichen technologischen Vorsprung gegenüber der europäischen Konkurrenz. Obschon man auch in den USA Anfang der fünfziger Jahre noch weit von einem ersten Sättigungsgrad auf dem s/w-Fernsehmarkt entfernt war, beschäftigte die Rundfunkfachleute längst ein anderes Thema: das Farbfernsehen! Beide Faktoren erklären, warum man die - aus US-amerikanischer Perspektive - leidige europäische Diskussion um eine s/w-Zeilennorm recht gelassen beobachtet hat. 
7) Die europäischen Länder, die sich aktiv für eine kommunikative Lösung des Standardisierungsproblems, das heißt auf Verhandlungsbasis im Rahmen der zuständigen internationalen Behörden (CCIR/EBU/OIR), stark gemacht haben, waren jene Länder, die aufgrund ihrer industriellen Struktur keine wirtschafts- oder industriepolitische Macht ausüben konnten. Besonders die kleinen, mehrsprachigen Länder (Schweiz, Luxemburg und Belgien) versuchten vergeblich, auf eine Kompromißlösung hinzuarbeiten. Das Scheitern dieser Bemühungen traf diese Länder besonders hart, da die Mehrnormigkeit auf Sender- und Empfängerseite hohe Kosten verursachte.

8) Wie das Beispiel Frankreichs zeigt, spielten in der nationalen Entscheidung für einen $\mathbf{s} / \mathbf{w}$-Standard neben politischen und wirtschaftlichen Fakten auch persönliche Interessen eine Rolle. Die engen Kontakte zwischen Vertretern der Firma Radio-Industries (v.a. Henri de France) und den Verantwortlichen der RTF (v.a. Wladimir Porché) haben dem 819-Zeilensystem letztlich zum Durchbruch verholfen. Insgesamt hatte man sich in Frankreich im Gegensatz zu den anderen europäischen Ländern aber auf das hochauflösende Fernsehen als der Zukunftstechnik festgelegt. Diese technologische Weichenstellung war auch Ausdruck eines französischen Selbstverständnisses, das sich durch höchste Qualitätsansprüche (= technische Elite) definierte. 


\subsection{Die Normung der Grundparameter für das Farbfernsehen in Europa 1955-1961}

Waren die Bemühungen um einen einheitlichen s/w-Fernsehstandard in Europa mit der Stockholmer CCIR-Konferenz des Jahres 1952 de facto gescheitert, führten die Fortschritte des internationalen Programmaustausches im Rahmen der Eurovision dazu, daß den Verantwortlichen der europäischen Rundfunkanstalten der enorme Aufwand der Normwandlungen und die damit verbundenen Mehrkosten bei gleichzeitiger Einbuße an Bildgüte immer bewußter wurde. Sehr bald mehrten sich daraufhin die Stimmen, die vor einer Wiederholung einer solchen Fehlentwicklung im Bereich der noch zu definierenden Farbfernsehnormen warnten. Würde man sich nicht auf eine einheitliche Farbfernsehnorm einigen können, so der Tenor, würden die Schwierigkeiten für einen internationalen Programmaustausch im Farbfernsehen "praktisch unüberwindlich ${ }^{141}$ sein. Auf der CCIR-Zwischentagung in Brüssel im Frühjahr 1955 widmete sich die Studienkommission XI (Fernsehen) daraufhin erstmals dem Thema Farbfernsehen. »Unausgesprochen blieb dabei die Hoffnung «, so berichten Mitarbeiter des Fernmeldetechnischen Zentralamtes der Bundespost in Darmstadt, »daß man auf dem Weg über die gemeinsame Farbnorm schließlich doch noch zu einer gemeinsamen s/w-Norm kommen würde ${ }^{142}$. Diese Hoffnungen sollten sich jedoch bald als illusorisch herausstellen. Dennoch gelang es, einen Katalog von technischen Fragen zu erarbeiten, der als Arbeitsplan für die zukünftigen Treffen der Studiengruppe XI dienen sollte. Folgende Punkte sollten bei der Diskussion über eine europäische Farbfernsehnorm berücksichtigt werden: befriedigende Bildgüte; sparsamer Gebrauch der Bandbreite; zuverlässige Empfänger mit tragbaren Kosten; Betrieb von Studio-, Sender- und Übertragungseinrichtungen; Empfindlichkeit gegen Störungen; Kompatibilität mit bestehenden Schwarzweißfernsehern; Frequenzplanung; internationaler Programmaustausch sowie die Unterschiede zwischen den Bandbereichen I/III und IV/V.

In Brüssel wurde zudem erstmals die Idee verfochten, daß man das farbige Fernsehen in Europa auf einen besonderen Wellenbereich beschränken sollte, und zwar den Dezimeter-Wellenbereich, der auf der Funkverwaltungskonferenz von Atlantic City 1947 den Bändern IV und V (470 bis $790 \mathrm{MHz}$ ) zugeschrieben worden war. Dieser Wellenbereich war bislang nicht für das Schwarzweißfernsehen in Anspruch genommen worden. Bis auf weiteres ver-

141 Einen ausführlichen Überblick über die frühen Bemühungen um einen europäischen Farbfernsehstandard im Rahmen der CCIR und EBU/OIR bietet der Aufsatz des Oberpostdirektors und Leiter des Fernmeldetechnischen Zentralamtes (FTZ) in Darmstadt, Johannes MüLLER, Die internationalen Bemühungen um eine europäische Farbfernsehnorm.

142 J. Müller, F. KIRSChSTEIN, K. O. SCHMIDT, Die Bemühungen des CCIR um eine europäische Norm für das farbige Fernsehen, in: Nachrichtentechnische Zeitschrift 1 (1957) S. 20. 
einbarte man daher ein »Stillhalteabkommen« was die Nutzung dieses Wellenbereichs betraf, um einer potentiellen Einigung für den Farbfernsehstandard keine Hindernisse in den Weg zu stellen.

$\mathrm{Da}$ in den meisten Ländern jedoch noch keinerlei Erfahrungen im Bereich des Farbfernsehens vorhanden waren, beschloß man, eine international besetzte Gruppe auf Studienreise in die USA zu entsenden. Diese sollte auch in Frankreich, England und den Niederlanden Station machen, die als einzige europäische Länder bereits gewisse Grundlagenarbeiten im Bereich des Farbfernsehens vorweisen konnten. Vom 6. März bis 12. April 1955 besuchte die Studiengruppe die USA, das einzige Land, das seit 1954 regelmäßig Farbsendungen nach dem NTSC-System ausstrahlte. Sämtlichen Größen der amerikanischen Rundfunkindustrie wurde ein Besuch abgestattet: den Laboratorien der Hazeltine Corporation, dem Forschungsinstitut der RCA in Princeton, den Bell-Laboratories von AT\&T in Murray-Hill sowie der Philco-Corporation in Philadelphia. Hinzu kam der Besuch mehrerer Sendeanstalten und Produktionsstätten der oben genannten Firmen. Im Reisebericht der drei Mitarbeiter des FTZ in Darmstadt (Kirchstein, Müller und Schmidt) hieß es:

Faßt man alle Eindrücke von den Vorfürungen in den USA zusammen, so kann man vielleicht folgendes sagen: Es ist grundsätzlich möglich, mit dem NTSC-System gute, farbige Bilder zu übertragen, ohne daß im Äther ein breiteres Frequenzband belegt wird als bei Schwarz-WeißÜbertragungen. Diese farbigen Sendungen sind mit den zur Zeit durchgeführten schwarz-weißen >verträglich،. Ein entsprechender farbiger Fernseh-Rundfunk muß jedoch zur Zeit mit gewissen Anfangsschwierigkeiten rechnen. Außerdem sind die derzeitigen Aufnahmegeräte (mit den drei Super-Orthicons) und die Wiedergaberöhren (in der Form der shadow-mask-tube) noch sehr kompliziert, so daB hier mit einer technischen Weiterentwicklung gerechnet werden muB ${ }^{143}$.

Eine solche »Anfangsschwierigkeit« - die sich später jedoch als ernsthaftes "Dauerproblem« des NTSC-Systems herausstellen sollte - war, daß die Rundstrahlung farbiger Sendungen ernsthafte Probleme bereitete, was der Phasenverdrehung des amplitudenmodulierten Farbhilfsträgers angelastet wurde $^{144}$. Vom 3. bis 7. April hielt sich die Studiengruppe in England auf, wo u. a. die Unternehmen Marconi und Electrical Music Industries (EMI) sowie die Forschungslaboratorien der BBC besichtigt wurden. Interessant an den englischen Vorführungen war, da $B$ hier Farbfernsehsendungen nach dem NTSC-System sowohl in der 405- als auch 625-Zeilennorm vorgeführt wurden und auch deren gegenseitige Umwandlung realisiert wurde. Im Unterschied zu den Vorführungen in den USA und England zeigten die Reisen nach Holland und Frankreich Alternativen zum NTSC-System auf. In den

143 Ibid. S. 24.

144 »Echter Rundfunk-Empfang [...] wirkte nicht immer überzeugend. [...] Die Farben waren blaß und unnatürlich und auf den verschiedenen im gleichen Raum aufgestellten Empfängern ganz verschieden «, ibid. S. 22. 
Niederlanden führten die Ingenieure der Philips-Forschungslaboratorien in Eindhoven ihr »Two-Sub-Carrier-System « (TSC-System) vor, das im Gegensatz zum NTSC-System zwei statt einen Farbhilfsträger im gleichen Kanal übertrug ${ }^{145}$. Aber auch bei Philips studierte man eindringlich das NTSC-System, das man der Studiengruppe ebenfalls in Experimentalvorträgen vorführte. Den Abschluß der Studienreise bildete der Besuch in Frankreich. Während alle anderen Länder sich mehr oder weniger eng an das NTSC-System anlehnten, ging man in Frankreich eigene Wege ${ }^{146}$. Die bereits bekannte "Société Radio Industries « führte ein von Henri de France entwickeltes Farbfernsehsystem auf 409-Zeilenbasis vor. Die »Laboratoires d'électronique et de physique appliquées zeigten ein von Prof. Georges Albert Boutry entwickeltes System namens "Double Message«, und die Ingenieure der RTF stellten ein System des französischen Ingenieurs Georges Valensi vor. Alle drei französischen Systeme wurden aber von der Studiengruppe als »im Zustand der Laborentwicklung « beschrieben. Ihre Vergleichbarkeit mit dem NTSC-System wurde daher stark in Frage gestellt.

Als die Studiengruppe auf der nächsten CCIR-Vollversammlung im August 1956 in Warschau über die Ergebnisse der ausgiebigen Studienreise berichtete, erklärten die "Gerber-Länder« (also jene Länder, welche die 625 -Zeilennorm bei einem Bild-/Tonträgerabstand von $5,5 \mathrm{MHz}$ für ihr Schwarzweißfernsehen als Standard definiert hatten), daß sie von der Brauchbarkeit des NTSC-Systems prinzipiell überzeugt und daher bereit seien, »die Konzeption dieses Systems als Grundlage für die europäische Norm « anzunehmen. Auch die niederländische Postverwaltung erklärte sich bereit, im Interesse einer einheitlichen Lösung auf die Weiterentwicklung des TSC-Systems der Firma Philips zu verzichten. Die englische Delegation stimmte zwar der technischen Beurteilung des NTSC-Systems zu, hielt aber die Entscheidung über die Definition einer Zeilenzahl für das Farbfernsehen in Europa für verfrüht. Dagegen hielt die französische Delegation auch eine definitive technische Beurteilung des NTSC-Systems für voreilig, solange die in Frankreich laufenden Untersuchungen der Systeme »Henri de France«, »Double Message« und »Valensi« nicht beendet seien. In einem abschließenden Kommuniqué der Konferenz hieB es daraufhin: "Zur Zeit ist nur ein Farbfernsehsystem - nämlich das NTSC-System - so weitgehend erprobt,

145 Der Vorteil dieses Systems gegenüber dem NTSC-System war die geringere Empfindlichkeit gegenüber den Phasendrehungen der Farbhilfsträger verbunden mit einer einfacheren Empfängerschaltung, die durch den Wegfall der »synchronen Demodulation« des Hilfsträgers erreicht wurde. Nachteil war, daß die einfachere Demodulation der beiden Hilfsträger stärkere Rauschstörungen verursachte und die Amplitudenmodulation der Hilfsträger eine besondere Kunstschaltung erforderte. Siehe Müller, KIRSChSteIn, Schmid, Die Bemühungen des CCIR um eine europäische Norm für das farbige Fernsehen, S. 25.

146 Ibid. S. 26. 
$\mathrm{da} B$ es für eine europäische Norm in Betracht gezogen werden kann. Dies soll aber nicht ausschließen, daß eine andere Norm erprobt und angenommen wird, wenn ihre Erprobung mit befriedigenden Ergebnissen abgeschlossen

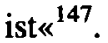

Trotz dieser relativ vagen Abschlußerklärung waren sich die meisten Teilnehmer der Konferenz darüber im klaren, daß für die zukünftige Planung des Fernsehsendernetzes und für die Organisation des internationalen Programmaustausches folgende Grundparameter verbindlich definiert werden mußten: erstens die Zeilenzahl, zweitens das HF-Kanalraster und drittens die Farbhilfsträgerfrequenz. Diese »Normung der Grundparameter« war von zentraler Bedeutung und stellte die Diskussion um die jeweiligen Farbfernsehsysteme vorerst in den Schatten, die sich snur ` in der Modulationsart des Farbhilfsträgers unterschieden.

Um dies zu erreichen, wurde außerhalb des offiziellen CCIR-Rahmens von den Delegationen aus der Schweiz, Italien, den Niederlanden und der Bundesrepublik Deutschland eine "Arbeitsgemeinschaft für das Farbfernsehen « unter dem Vorsitz des Schweizers Dr. Gerber (»Gerber-Ausschuß«) gebildet. Der Ausschuß setzte sich pro Land aus je einem Vertreter der Postverwaltung, der Industrie und der Rundfunkanstalten zusammen. Ziel dieser Arbeitsgruppe war es, »im kleinen Kreise, unabhängig vom CCIR, aber dennoch um die Arbeit des CCIR zu erleichtern«, die technischen Fragen und Probleme, die mit einer zukünftigen europäischen Farbfernsehnorm zusammenhingen, zu diskutieren und zu untersuchen. Gegebenenfalls sollten auch gemeinsame Übertragungsversuche durchgeführt werden ${ }^{148}$.

Natürlich fällt auf, daB in dieser Arbeitsgruppe weder Franzosen noch Briten vertreten waren. Wie ein Brief des damaligen Technischen Direktors des NDR, Dr. Werner Nestel, an den Intendanten des Hessischen Rundfunks Beckmann vom 7. Mai 1955 zeigt, war man auf deutscher Seite zuversichtlich, mit Herrn Dr. Gerber die richtige Person für diese schwierige Aufgabe beauftragt zu haben: »Da Herr Dr. Gerber seinerzeit mit so großem Geschick die 625-Zeilen-Gerber-Norm in einer so großen Zahl von Ländern zur Annahme gebracht hat, können wir annehmen, daß sein Vorsitz die besten Voraussetzungen bietet, da $B$ auch die zukünftige Farbnorm in unserem Sinne günstig gewählt wird«, so Nestel ${ }^{149}$.

Tatsächlich avancierte die Arbeitsgruppe unter der Leitung des Schweizer Postdirektors Gerber in der Folgezeit zu einem zentralen Gremium, das die Notwendigkeit der Standardisierung der Parameter für das europäische Farb-

147 MülLLE, Die internationalen Bemühungen um eine europäische Farbfernsehnorm, S. $229 \mathrm{f}$.

148 Ibid. S. 230.

149 Brief von Dr. Werner Nestel an Herm Intendant Beckmann/Hessischer Rundfunk, 7. Mai 1955, 4 maschinegeschriebene Seiten, hier S. 3, in: Historisches Archiv des Westdeutschen Rundfunks, Bestand Brack, Femseh-Kommission der ARD 1952-1955, VSTO 119. 
fernsehen immer wieder betonte und mit aller Kraft auf eine solche Einigung hinarbeitete. Nicht unerwähnt bleiben sollte, daß in diese Zeit der »Normung der Grundparameter « auch die Verhandlungen zur Gründung der Europäischen Wirtschaftsgemeinschaft fielen, die ihren Abschluß in den Römischen Verträgen 1957 fanden. Das Thema Farbfernsehen stand zwar nicht auf der Verhandlungsliste in Rom, die Bemühungen um einen harmonisierten europäischen Wirtschaftsraum wirkten sich indirekt aber auch auf die internationale Ordnung des Rundfunks sowie die europäische Rundfunkindustrie aus. So versuchten die EWG-Länder noch vor der CCIR-Tagung in Moskau im Juni 1958 einen gemeinsamen Standpunkt über ein HF-Kanalraster für das Schwarzweißfernsehen und das Farbfernsehen zu erarbeiten. Man einigte sich schließlich auf die Kanalbandbreite von $10 \mathrm{MHz}^{150}$.

In Moskau konnte man sich zwar nicht auf die Zahl von 625 Zeilen einigen, aber es zeichnete sich erstmals eine gewisse Bereitschaft ab, sich auf eine Bandbreite von $8 \mathrm{MHz}$ für das Farbfernsehen verständigen zu können. Der nächste entscheidende Schritt wurde auf der IX. Vollversammlung der CCIR in Los Angeles im März 1959 getan. Mit Ausnahme von Frankreich bestand Konsens darüber, das Farbfernsehen generell auf die Bänder IV und $\mathrm{V}$ mit einem HF-Kanalraster von $8 \mathrm{MHz}$ festzulegen ${ }^{151}$. Entscheidend für diese Wende war die Mitteilung der britischen Delegation, daß sie - vorbehaltlich der Entscheidung des Television Advisory Committee - bereit sei, in den Bereichen IV und V die 625-Zeilennorm bei einem Bild-/Tonträgerabstand von 6,5 $\mathrm{MHz}$ einzuführen, also im Bereich des Farbfernsehens ihre 405-Zeilennorm zugunsten der Gerbernorm aufzugeben. Selbst Frankreich, das wegen der Möglichkeit einer Kompatibilität mit dem 819-Zeilensystem auf der höheren Bandbreite von $16 \mathrm{MHz}$ pochte, ließ erstmals eine gewisse Bereitschaft erkennen, für das Farbfernsehen auf ein 625-Zeilensystem überzugehen.

Trotz dieser positiven Entwicklungen hinterließ die CCIR-Tagung in Los Angeles keine sehr positiven Eindrücke von der "praktischen Realität« des amerikanischen Farbfernsehens. Es fanden weder Vorführungen von seiten der großen network-stations statt, noch waren in Geschäften, Restaurants oder Bars viele Empfänger zu sehen. Das "große Geschäft« mit den Farbempfängern setzte auch in den Vereinigten Staaten erst ab 1962 ein. Die Gründe für den zähen Start des Farbfernsehens in den USA sind klar: Die amerikanische Rundfunkindustrie war bis Anfang der 1960er Jahre voll damit ausgelastet, die US-amerikanische Bevölkerung mit $\mathrm{s} / \mathrm{w}$-Empfängern zu versorgen. Erst als der s/w-Markt gesättigt war, konnte der Vertrieb der wesentlich teureren Farbempfänger langsam in Angriff genommen werden.

150 MüLLER, Die internationalen Bemühungen um eine europäische Farbfernsehnorm, S. 231.

151 Allerdings gingen auch in den Gerber-Ländern die Meinungen über den Bild-/Tonfrequenzabstand auseinander. 
Zudem war das Programmangebot an Farbsendungen sehr beschränkt. So strahlten 1956 nur drei der sieben in New York befindlichen Fernsehsender circa 40 Stunden Farbprogramme pro Monat aus. Die Zahl der Farbfernsehempfänger im Sendegebiet belief sich auf schätzungsweise 50000 Appara$\mathrm{te}^{152}$.

Wurde der schleppende Start des Farbfernsehgeschäftes in den USA von europäischen Beobachtern auch der technischen Unausgereiftheit des NTSCSystems zugesprochen, glaubt John Wentworth, daß die Gründe »in der nur schwer erfaßbaren Käuferpsychologie und im Prinzip der freien Marktwirtschaft « zu suchen seien, nicht aber in systembedingten technischen Mängeln ${ }^{153}$. Trotz der $»$ neuen Dimension« Farbe sei das Farbfernsehen lediglich eine Verfeinerung der s/w-Technik, nicht aber so revolutionär neu wie das Schwarzweißfernsehen. Wentworth macht sogar einen »circulus vitiosus « aus, in dem die Einführung des Farbfernsehens gefangen gewesen sei: Da das Fernsehen in den USA vorwiegend durch Werbung finanziert wurde, die Produktion von Farbspots aber wesentlich teurer war als die von s/w-Spots, zudem noch viel mehr s/w-Zuschauer vorhanden waren als Farbfernsehbesitzer, konnte das Farbfernsehen nur schleppend in Gang kommen. Aus diesem "Dilemma«, so Wentworth, sei man erst durch einen »kühnen wirtschaftlichen Anstoß« herausgekommen, der das historische Verdienst der RCA sei. Die RCA habe vor allem auf dem Gebiet der Farbfernsehröhre geforscht und in Kundendienstwerkstätten ausgiebig über das Farbfernsehen informiert und geworben. Hinzu kam, daß die zögernde Programmproduktion den Kauf von Farbgeräten nicht attraktiv machte. Die Voraussetzung für eine erhöhte Produktion der kostspieligen Farbprogramme war wiederum an das Vorhandensein einer größeren Zuschauerzahl, das heißt von Farbgerätebesitzern gebunden.

Doch zurück zu den europäischen Bemühungen. Nachdem in Los Angeles deutliche Schritte auf einen »common sense « hin gemacht worden waren, kam man im Oktober des gleichen Jahres erneut zusammen. Unter der Leitung des Schweden Erik Esping, der auch Vorsitzender der Studiengruppe XI des CCIR war, kam man in Genf zu dem Ergebnis, daß als Grundlage für die Frequenzplanung in den Bereichen IV und V, insbesondere für das Farbfernsehen, nur noch Normen mit 625 Zeilen und einem einheitlichen HF-Raster von $8 \mathrm{MHz}$ berücksichtigt werden sollten. Auf die Farbträgerfrequenz konnte man sich aber nicht verständigen. Dies geschah erst auf der "Expertentagung « in Cannes im Februar 1961, als man den britischen Vorschlag von 4,43 MHz bei einheitlichem HF-Abstand von $8 \mathrm{MHz}$ festlegte. Dieser Vorschlag ging als Arbeitsgrundlage in die europäische Rundfunkkonferenz von

152 MÜLlER, Die internationalen Bemühungen um eine europäische Farbfernsehnorm, S. 226f.

153 John W. WENTWORTH, Zusammenfassung der praktischen Erfahrungen mit dem Farbfernsehen in den Vereinigten Staaten, in: Rundfunktechnische Mitteilungen 8 (1964) 4 S. 236-244. 
Stockholm im Juni 1961 ein, wo sowohl Frankreich als auch Großbritannien erklärten, auch ihre s/w-Norm an diese neuen Charakteristika anpassen zu wollen. Die noch übriggebliebenen Unterschiede im Bereich der Ton-/Bildfrequenzabstände wurden im Vergleich zur erreichten Harmonisierung der anderen Parameter als »nicht sehr gravierend« angesehen. War die Entscheidung in Stockholm ein Meilenstein in der Geschichte der Harmonisierung der Fernsehnormen in Europa, bedeutete diese Entscheidung für Frankreich und Großbritannien, daß enorme finanzielle Belastungen auf sie zukommen würden. Die Umstellung von Studio-, Sender- und Empfangstechnik auf eine neue Zeilennorm war ein kostspieliges Unterfangen, das jahrelange Übergangslösungen mit entsprechenden »Überbrückungstechniken« erforderte.

Welche tiefgreifende Bedeutung die Entscheidung für die Einführung einer von der vorhandenen Norm abweichenden Zeilennorm für ein Land hatte, läßt sich beispielhaft an der Situation Englands demonstrieren. Im Juli 1960 wurde in England im Auftrag des Postmaster General das sogenannte »Pilkington Committee« unter Vorsitz von Sir Harry Pilkington gegründet. Aufgabe des Kommittees war die Erarbeitung eines Berichtes, der der englischen Regierung beziehungsweise dem Postminister u.a. eine Empfehlung über die auszuwählende Zeilennorm im Bereich des Farbfernsehens machen sollte ${ }^{154}$. Ähnlich wie in Frankreich gingen die Meinungen über die zu adoptierende Zeilennorm quer durch alle Lager (Postverwaltung, BBC und Rundfunkindustrie) ziemlich weit auseinander.

Die BBC richtete am 9. Dezember 1960 ein Schreiben an den Postmaster General, in dem sie die britische Regierung dazu aufforderte, für eine baldige Einführung des Farbfernsehens auf der Basis der bestehenden 405-Zeilennorm zu stimmen. Sir Carlton Greene, Generaldirektor der BBC, verwies in einem Schreiben darauf, daB die BBC bereits seit 1955 erfolgreich regelmäßige Farbfernsehversuche nach dem amerikanischen NTSC-System durchführe. »The $\mathrm{BBC}$ «, so Greene, »which started its television service well in advance of the rest of the world, does not believe that it should lag further behind the United States, Japan and the Soviet Union “ ${ }^{155}$. Neben der politisch-symbolischen Bedeutung, die dem Farbfernsehen laut Greene zukam, stellte sich die BBC argumentativ auf die Konsumentenseite. Da eine europäische Entscheidung im Sinne eines einheitlichen Standards nach Meinung der BBC nicht zu erwarten war, bedeutete das Warten auf eine solche Entscheidung, daß England den fernsehtechnischen Vorsprung, den es damals im europäischen Kontext hatte, verlieren würde. Eine von der bestehenden

154 Zur Geschichte und Bedeutung des Pilkington-Kommitees siehe Asa BrIGGs, The History of Broadcasting in the United Kingdom, Bd. 5: Competition, Oxford 1995. Hier besonders das Kapitel 4, »Under Review: Pilkington«, S. 257-308.

155 Brief von Hugh Carlton Greene (Chairman of the BBC) an German (G.P.O. Headquarters), London, 9.12. 1960, in: Public Record Office (PRO, London), Signatur HO 256/386. 
405-Zeilennorm abweichende Entscheidung hätte zudem zur Folge gehabt, $\mathrm{da} ß$ nur unter großem finanziellen Aufwand eine Benachteiligung der damaligen $\mathrm{s} / \mathrm{w}$-Fernsehbesitzer ausgeschlossen werden konnte ${ }^{156}$.

Ein scharfer Gegner der BBC war die englische Firma PYE, führender Fernsehgerätehersteller in England. Charles Stanley, Direktor von PYE, sah mit der Einführung eines Farbfernsehsystems auf 405-Zeilenbasis alle Exportchancen schwinden. Ablehnend gegenüber dem Vorschlag der BBC äuBerte sich auch die Independant Television Authority (ITA), die staatliche Aufsichtsbehörde der privaten Fernsehkonkurrenz der BBC in England. Für die Beibehaltung des 405-Zeilensystems sprach sich dagegen mehrheitlich der Verband der englischen Rundfunkindustrie BREMA (British Radioelectronics Manufacturers Association) aus ${ }^{157}$.

Da die BBC behauptete, binnen eines Jahres Farbfernsehen auf der Basis des NTSC-Systems angepaßt an die britische 405-Zeilennorm einführen zu können, stand der britische Postminister Wedgwood Benn vor der schwierigen Entscheidung, entweder dem Druck der BBC und der englischen Rundfunkindustrie nachzugeben, oder aber den Bericht des Pilkington-Committees abzuwarten. Pilkington hatte gleich zu Beginn seiner Arbeit klar gemacht, $\mathrm{da} ß$ er es für sinnvoll ansehe, die Entscheidung im Bereich des Farbfernsehens an die europäischen Verhandlungen im Rahmen der CCIR zu binden. In einem ersten Arbeitsbericht des Pilkington-Committees hieß es:

Further, we feel that the question of the definition standards to be adopted in Bands IV and V should not be determinated before a decision is reached in colour. If 625 -line are to be introduced it is naturally desirable that colour when introduced should use this standard. It would not be helpful to introduce colour on 405-line standards in Bands I and III in the meantime ${ }^{158}$.

Eine Entscheidung zugunsten des 625-Zeilensystems bedeutete, dessen war man sich im Postministerium bewußt, eine Verschiebung des Einführungstermins des Farbfernsehens bis mindestens 1965. Dennoch teilte das Postministerium der BBC am 10. Mai 1961 mit, daß man die Initiative zur frühen Einführung des Farbfernsehens leider ablehnen müsse. Zwar habe die BBC einige gute Gründe für den frühen Einführungstermin angeführt, aber die Exportchancen für ein 405-Zeilen Farbfernsehsystem seien gleich Null (außer nach Irland). Auch müsse man vor einer definitiven Entscheidung die Empfehlungen des Pilkington-Committees abwarten. Man könne jetzt nicht Leute

156 I. R. Atkins, A. R. Stanley, S. N. Watson, A New Survey of the BBC Experimental Colour Transmissions, BBC Engineering Division Monograph Number 32, October 1960.

157 Brief von Mr. Judd (General Post Office) an Wedgwood Benn (Postmaster General), London, 1. Mai 1961, 3 maschinegeschriebene Seiten, hier S. 2, in: PRO, Signatur HO 256/386.

158 Siehe »Postmasters General's Questions on Colour Television«, dreiseitiger Frage- und Antwortkatalog, der die pro- und contra-Argumente aus der Perspektive der britischen Postverwaltung zusammenfaßt, ohne Datum (wahrscheinlich Anfang Mai 1961), in: PRO, Signatur HO $256 / 386$. 
zum Kauf eines Farbfernsehers ermuntern, wenn in zwei Jahren die Zeilennorm verändert würde ${ }^{159}$.

Als das Pilkington-Committee am 27. Juni 1962 seinen 340-Seiten dicken Bericht vorlegte, fiel die Entscheidung zugunsten eines einheitlichen Fernsehbetriebes nach dem 625-Zeilenstandard sowohl für das SchwarzweiBfernsehen als auch für das zukünftige Farbfernsehen aus. Für die Rundfunkindustrie in England bedeutete dies, daB sie ihre Produktion auf teurere und kompliziertere Zweinormenempfänger umstellen mußten. Die Mehrkosten der Produktion mußten zum Teil auf die Konsumenten abgewälzt werden, die für die Mehrnormenempfänger tiefer in die Tasche greifen mußten. Als dann 1963 in Europa ernsthafte Diskussionen darüber begannen, vielleicht ein anderes Farbfernsehsystem als das amerikanische NTSC-System einzuführen, war die Geduld der englischen Rundfunkgerätehersteller endgültig am Ende. In einem Brief des einflußreichen Geschäftsführers der Rank-Bush Murphy Ltd., Saward, an den Sekretär des Television Advisory Committee, Judd, vom 13. Mai 1963 heißt es:

At the present time the set manufacturing industry is, as you know, heavily committed by the introduction of the complicated $405 / 625$ line, VHF/UHF, dual standards receiver. To introduce a third standard before the first change is properly digested could prove quite desastrous to the set manufacturers. [...] It is our considerated opinion that the adoption of SECAM or PAL must inevitably put back the introduction of colour by at least two years ${ }^{160}$.

Doch greifen wir hier schon der nächsten Etappe des komplexen Verhandlungsprozesses in der europäischen Farbfernsehdebatte vor. Ähnlich kompliziert wie in Großbritannien gestaltete sich auch die Diskussion über die europäische Zeilennorm für das Farbfernsehen in Frankreich ${ }^{161}$. Schon beim Wechsel vom 441-Zeilensystem auf die 819-Norm hatte sich auf Seiten der wenigen Fernsehbesitzer Widerstand geregt. Sie fürchteten um die Sicherheit ihrer Investition und gründeten gar einen eingetragenen Verein namens „Groupement de défense des téléspectateurs 441-lignes«, um gegen die Umstellung auf 819 Zeilen zu protestieren ${ }^{162}$. Die Entwicklung des Fernsehens im Bereich des internationalen Programmaustausches (Gründung der Eurovi-

159 „I know I do not need to remind you that the Television Advisory Committee felt very firmly that colour should only be brought in using the line standards to be ultimately adopted for black and white transmissions, and that a decision about colour should follow a decision on line standards. « Brief von Bevins (HPG) an Forde (BBC), London, 10. Mai 1961, in: PRO, Signatur HO 256/386.

160 Brief von Saward (Rank Bush Murphy Ltd.) an Judd (Secretary of the Television Advisory Committee), London, 13. Mai 1963, in: PRO, Signatur HO 256/386.

161 »Unification des normes de télévision/Groupe de travail pour l'unification des normes de télévision en France, FNIE, 18.02. 1965, in: ORTF, Les normes de télévision en France (internes Studiendokument der ORTF), in: Archives du CHTV/INA.

162 In einem Dossier des RTF-Bestandes in den Archives nationales finden sich zahlreiche Klagebriefe von Leuten, die nach der Beendigung von Sendungen nach dem 441-Zeilensystem im 
sion) und der langsame Anstieg der Fernsehzuschauer in Frankreich ließen dort bereits 1955 die Frage eines zweiten Fernsehprogramms aufkommen. Im August 1955 legte daraufhin das vom französischen Parlament eingesetzte »Comité d'études des problèmes de presse« einen »Rapport technique sur les possibilités de création d'une seconde chaine de télévision en France« vor, in dem die Frage der zukünftigen Zeilennorm für das Farbfernsehen als dringendes Problem erkannt wurde. Hypothetisch wurden drei Möglichkeiten durchgespielt, die sich bei der Einführung des Farbfernsehens für Frankreich ergeben könnten: a) ein mit dem bestehenden 819-Zeilensystem kompatibles Farbfernsehsystem; b) Beibehaltung der 819-Zeilennorm für s/w-Fernsehen und Einführung eines Farbfernsehsystems auf der Basis eines 625-Zeilensystems; c) Schwarzweiß- und Farbfernsehen auf der Basis eines zukünftigen europäischen Farbfernsehstandards mit 625 Zeilen.

Tatsächlich wurden in Frankreich alle drei Varianten als jeweils einzuschlagende Wege in chronologischer Reihenfolge verfolgt. 1958 war man sich einig, daß die Zukunft des französischen Fernsehens sowohl im Bereich der Farbe als auch beim Schwarzweißfernsehen auf der alleinigen Basis des 819-Zeilensystems zu gestalten sei. Doch schon ein Jahr später überwog die b-Variante bei den Technikern der RTF. Auf der CCIR-Tagung in Stockholm erreichte den französischen Delegationsleiter am 24. Mai 1961 die endgültige Weisung des französischen Informationsministers, für die Einführung des 625-Zeilensystems als Basis des Fernsehens in den Bändern IV und V zu stimmen ${ }^{163}$. Wie das "Pilkington Committee in Großbritannien kam 1955 auch das »Comité d'études des problèmes de presse « in Frankreich zu dem Schluß, daß das Problem der zukünftigen Zeilennorm im Bereich des Farbfernsehens Rückwirkungen auf das bestehende Schwarzweißfernsehen haben werde, weshalb eine Entscheidung in diesem Bereich verfrüht erscheine:

On peut certes admettre comme base que le second réseau, étant donné son époque de mise en chantier, doit prévoir la couleur. Mais avec quel système? Cette question conditionne également le système en noir et blanc avec lequel débuterait l'exploitation. Il n'est pas possible actuellement de le dire, en absence de toute indication sur le futur système européen. Mais comme, en fait l'installation du second réseau doit avoir lieu dans les bandes IV et V, et comme notre pays s'est engagé à ne pas les occuper par un service régulier avant que l'on ait quelques précisions

Jahre 1956 (ein Brand auf dem Eiffelturm hatte den Sender zerstört) einen finanziellen Ausgleich vom Staat verlangten. Siehe Archives nationales, Signatur F-41/2302.

163 "Je vous confirme que le Gouvernement a, au cours d'un récent Conseil des Ministres, décidé d'adopter la définition de 625 lignes pour les émissions de télévision dans la bande de fréquences comprise entre 470 et $960 \mathrm{Mc} / \mathrm{s}$ (bandes IV et V) Monsieur le Chef de la Délégation Française à la Conférence de Stockholm, 24. mai 1961, in: Archives nationales, Signatur F-41/2303. 
sur le système européen, ces deux conditions coincident pour donner un certain délai à la necessité de cette réponse ${ }^{164}$.

Wie in England bedurfte es der Erfahrungen im Bereich der Eurovision, des Drucks seitens einiger Rundfunkindustrieller sowie eines Umdenkens auf politischer Ebene, um die Entscheidung für das 625-Zeilensystem herbeizuführen. Bedeutete diese Entscheidung enorme finanzielle Mehrbelastungen für den französischen Staat als Träger der RTF - 1965 wurden die Kosten auf 25 bis 30 Milliarden alte Francs geschätzt ${ }^{165}$, war es gleichzeitig die RTF, die diese Entscheidung besonders befürwortet hatte. "Le choix du système à 625 lignes remet la France dans la compétition«, so das deutliche Urteil des Chefingenieurs der RTF, Louis Goussot ${ }^{166}$. Vor welche finanziellen und technischen Herausforderungen dieser Normwechsel die Radiodiffusion Télévision Française stellte, wird in einer Studie der Forschungs- und Entwicklungsdirektion der ORTF aus dem Jahre 1965 deutlich. In einem »Rapport sur les normes de télévision en France« werden sämtliche Probleme detailliert aufgelistet, mit denen die ORTF durch die Umstellung auf 625 Zeilen konfrontiert wurde. Gleich zu Beginn der Studie heißt es:

Le fait d'avoir adopté après la guerre des normes de télévision différentes de celles des autres pays européens place la France devant de très graves difficultés, particulièrement à l'occasion de l'introduction d'un service de télévision en couleur; l'obstacle immédiat réside dans l'impossibilité, dans l'état actuel de la technique, de réaliser un récepteur couleur pouvant reproduire les émmissions du premier programme avec le balayage de 819 lignes $^{167}$.

In enger Kooperation mit dem Verband der französischen Elektroindustrie (Fédération nationale des industries électroniques, FNIE) war die ORTF $1965 \mathrm{zu}$ dem Resultat gelangt, daß der Bau von Fernsehempfängern, die zugleich 819-Zeilen- und 625-Zeilenfernsehen umsetzen konnten, beim damaligen Stand der Technik unmöglich war. Diese technische Unmöglichkeit ließ für den Ausbau des Fernsehens bei der Einführung der Farbe (625-Zeilen) nur zwei mögliche Perspektiven zu. Die eine Perspektive (a) war, lediglich die Zeilenzahl für Schwarzweiß- und Farbsendungen zu harmonisieren. Die zweite Perspektive (b) ging darüber hinaus, indem sie den Fernsehbetrieb nicht nur mit 625-Zeilen vorsah, sondern ihn zudem komplett (d.h. auch den

164 Comité d'études des problèmes de presse, Rapport technique sur les possibilités de création d'une seconde chaîne de télévision en France, Paris, 12. August 1955, S. 11, in: Archives nationales, Signatur F-41/2303.

165 Note pour le Ministre de l'Information (A. Peyrefitte), Paris, 26.02.1965, in: Archives Diplomatiques du Quai d'Orsay, Affaires Économiques et Financières/Affaires Générales/Nr. 219a. 166 Goussor, Le choix des normes de la télévision en couleur en Europe, S. 10. Goussot bestätigte diese Einschätzung emeut im Interview mit dem Autor am 25. März 1999.

167 ORTF, Direction de l'équipement et de l'éxploitation: Les normes de télévision en France. Problèmes posés par l'introduction d'un service de télévision en couleur, Paris, Septembre 1965, Studienmappe mit 2 mehrseitigen Stellungnahmen zu diesem Thema von seiten der ORTF und der FNIE sowie drei mehrseitigen Anhängen, in: Archives du CHTV/INA. 
bestehenden s/w-Betrieb) auf den UHF-Bereich der Bänder IV und V verlegte. Während die ORTF die erste Perspektive als realisierbar ansah, vertrat die FNIE die zweite Hypothese, wrépondant à un souci de rationalisation et de logique tout à fait respectable«, wie es in der Studie hieß. Auf seiten der ORTF war man sich der Tragweite der zu treffenden Entscheidung bewußt:

La complexité de cette affaire est très sérieuse; aucune solution satisfaisante ne se dégage avec évidence; toutes présentent des inconvénients graves intéressant les relations de la F.N.I.E. avec sa clientèle ou de l'O.R.T.F. avec son public, c'est-à-dire dans les deux cas, les téléspectateurs. Cependant, une politique de la réception de télévision doit être esquissée: c'est l'avenir de la télévision en France qui en est bien la conséquence ${ }^{168}$.

Der Ernst der Lage wird klar, wenn man erkennt, welche Bedeutung die Entscheidung für die eine oder andere Variante für den französischen Rundfunk, die Rundfunkindustrie und den Fernsehzuschauer bedeutete. Entschied man sich für die komplette Umstellung auf den 625-Zeilenbetrieb, beließ aber den Programmbetrieb des Ersten Programms im VHF-Bereich (Bänder I bis III), bedeutete dies für die Rundfunkindustrie immer noch, daß die zukünftigen Farbfernsehempfänger »bi-standard «-Geräte sein mußten, da das erste Programm ( $\mathrm{s} / \mathrm{w}$ ) weiterhin in VHF, das zweite Programm (das zukünftige Farbprogramm) aber in UHF ausgestrahlt werden würde. Der Vorteil dieser Lösung aus Sicht der ORTF war, daß die bestehenden s/w-Empfänger relativ einfach auf 625-Zeilen s/w-Empfänger umgerüstet werden konnten. Dies war im Falle eines kompletten Bandwechsels (UHF-Betrieb) wesentlich aufwendiger. Die FNIE weigerte sich aber strikt, sich an den Kosten für eine solche Operation zu beteiligen, die sich mehrere Jahre hinziehen würde und deren Kosten sie auf 450 Millionen Francs schätzte. "La divergence fondamentale, entre l'industrie électronique et l'ORTF ${ }_{\kappa}$, so der Technische Direktor der ORTF, Claude Mercier, mréside dans la méthode d'exploitation entre l'apparition des premières émissions couleur $[\ldots]$ et l'échéance à préciser de l'abandon des normes 819 lignes ${ }^{169}$.

Um die Nachteile möglichst klein zu halten, die in der Phase zwischen dem Beginn der ersten Farbsendungen in den Bändern IV und V und dem Ende der Sendungen im 819-Zeilensystem auf die französischen Fernsehzuschauer zukamen, plädierte die FNIE für eine Verdopplung der s/w-Programme des Ersten Programms (Band I bis III, 819 Zeilen) auf Band IV und V (625 Zeilen). Dies bedeutete de facto die Etablierung eines Dritten Pro-

168 Claude Mrrcier, Rapport sur les normes de télévision en France, Paris, Septembre 1965, in: Ibid. S. 2.

169 Claude Mercier, Note sur les problèmes, particuliers à la France, posés par l'introduction d'un service de télévision en couleur. Cette note analyse des points de vue exprimés par la FNIE et l'ORTF à l'égard de ces problèmes au cours d'une réunion tenue le 3 février 1966 sous la présidence de M. Dupont, Directeur Général de l'ORTF, Paris, le 15 Février 1966, 7 maschinegeschriebene Seiten, hier S. 5, in: Archives du CHTV/INA. 
gramms (das Zweite sollte den Farbsendungen vorbehalten sein). Diese "Verdopplungsstrategie" wurde von den Verantwortlichen der ORTF auch unterstützt, allerdings sollte dies nur für den Sender der Hauptstadt Paris gelten. Die FNIE forderte jedoch eine Ausdehnung dieser Technik auf zehn weitere Sender, so daß mindestens $40 \%$ der Bevölkerung von dieser Lösung profitieren konnten.

Ohne weiter auf die unterschiedlichen Details in den Szenarien der ORTF und der FNIE eingehen zu können, sollte dieser kurze Einblick verdeutlichen, vor welche Probleme eine staatliche Rundfunkorganisation und die Rundfunkindustrie eines Landes bei einem Normwechsel gestellt wurden bzw. werden. Sowohl in Frankreich als auch in England kulminierte dieses Ereignis mit der Einführung der Farbfernsehtechnik, die den Fernsehingenieuren schon genügend Sorgen bereitete. In Frankreich kamen, wie zu zeigen sein wird, gewaltige strukturelle Probleme hinzu, da der staatliche französische Fernsehrundfunk ein neues organisatorisches Gesicht erhielt (Wandel von RTF zur ORTF) ${ }^{170}$. Die Entscheidung darüber, welches Farbfernsehübertragungssystem letztlich zum Einsatz kam, war vor diesem Hintergrund für die staatlichen Rundfunkanstalten in vielerlei Hinsicht »nebensächlich«. 


\section{5. »Zwischen Süßlila und Bonbonrosa«. Die europäischen Alternativen zum NTSC}

Obwohl - wie in Kap. 2.2. gesehen - das National Television System Committee das Problem eines kompatiblen Farbfernsehsystems »in genialer Weise ${ }^{171}$ gelöst hatte, zeigten sich beim Betrieb des Farbfernsehens in den USA bald gewisse Mängel. Probleme befeiteten vor allem so genannte Phasenverzerrungen des Farbhilfsträgers, die während der Übertragung der Signale durch künstliche (Hochhäuser) oder natürliche (Gebirge) Hindernisse hervorgerufen werden konnten. Da, wie gesehen, die Phase des Farbhilfsträgers auf optischer Ebene gleichzusetzen ist mit dem Farbton, hatten Phasenverschiebungen unschöne Farbtonänderungen des Fernsehbildes zur Folge. Ein ursprünglich blauer Himmel konnte so unter ungünstigen Empfangsbedingungen purpur oder cyan erscheinen. Dieser Umstand war dafür verantwortlich, $\mathrm{daB}$ die Abkürzung NTSC unter Fernsehingenieuren auch als Synonym für »Never Twice the Same Colour« gebraucht wurde. Um dieser Schwäche des Systems zu begegnen, stattete man die NTSC-Empfänger mit einem manuell zu bedienenden Regelknopf aus, mit dem der Fernsehzuschauer die Farbtonänderungen korrigieren konnte. Dieser Mechanismus veranlaßte manchen Kritiker dazu, sich ein wenig spöttisch über die Qualität des NTSC-Systems zu äußern. So schrieb der Redakteur der Fachzeitschrift Funkschau, Otto Limann: »Der amerikanische Farbfernseh-Teilnehmer kann selbst wählen zwischen Süßlila und Bonbonrosa. [...] Man kann also ein mittleres Rot willkürlich sowohl ins Rostbraune als auch in das Violette herüberziehen. Das läßt dem persönlichen Geschmack und dem Streit im Familienkreis über die angenehmste Farbe breitesten Spielraum ${ }^{172}{ }$.

In den USA kursierte der Witz, daB man beim Kauf eines Farbfernsehempfängers gleich einen Service-Ingenieur mitkaufen solle ${ }^{173}$. In der Zeitschrift »Radio-Electronics« sprach der Fernsehtechniker Homer Davidson seinen Kollegen Mut zu - sie sollten sich von den täglichen Anrufen entnervter Farbfernsehzuschauer nicht aus der Ruhe bringen lassen:

Will you please send a man over this moming to check my color TV? The color seems to come and go, and sometimes it's terrible. [...] It really isn't too difficult to trouble shoot color, if you progress logically through the set, function by function, stage by stage. Don't skip any links in the chain from the station signal to the CRT screen. And don't assume that something is okay just because it's supposed to be. Be sceptical, and you'll find the trouble ${ }^{174}$.

171 JAESCHKE, Europa vor der Einfuhrung des Farbfernsehens, S. 260.

172 Otto LimanN, Das Ziel der europäischen Farbfernsehnorm. Erste Farbfernsehtagung in der Öffentlichkeit, in: Funkschau 36 (1964) 14 S. 373.

173 E. R. FRIEDLÄNDER, Farbfernsehen in Großbritannien, in: Funk-Technik 23 (1968) 7 S. 231.

174 Homer Davidson, Exploring the Jungle of Color Troubles, in: Radio Electronics 38 (1967) 1 S. 58. 
Erstaunlich ist, daB diese technischen Mängel des NTSC-Systems auf den amerikanischen Kunden nicht so abstoßend wirkten, wie man es vermuten könnte. So berichtet John Wentworth darüber, daß es für den »Durchschnittsamerikaner« kein Problem sei, den Fernsehtechniker wegen diverser Probleme mit seinem Farbfernsehempfänger vier bis fünf Mal pro Jahr zu sich zu bestellen. Selbst beim s/w-Empfänger komme der Techniker im Durchschnitt zwei bis drei Mal pro Jahr ins Haus. Dies hatte auch mit dem Umstand zu tun, daß beim Kauf eines Farbempfängers in den USA in fast 50\% der Fälle gegen einen geringen Aufpreis ein Kundendienstvertrag abgeschlossen wurde, der die zukünftigen Serviceleistungen abdeckte. Die ständige Verbesserung der produzierten Empfänger machte das NTSC-System ab Anfang der sechziger Jahre aber zu einem durchaus stabilen System, das den Einsatz der "Farbregler « recht selten werden lieB ${ }^{175}$.

Vielleicht läßt sich aus diesen Aussagen auch ein grundsätzlicher Unterschied zwischen Amerikanern und Europäern herauslesen, was die Erwartungshaltung gegenüber technischen Artefakten angeht. An eine Technologie wird von den Amerikanern weniger der Anspruch der Perfektion als der Praktikabilität erhoben. Mit anderen Worten: Besser ist es, recht früh in den Besitz einer neuen Technologie zu gelangen und ihre $>$ Kinderkrankheiten $<$ in Kauf nehmen, als - wie im Falle des Farbfernsehens in Europa - dreizehn Jahre bis zur Einführung der neuen Technologie warten zu müssen. Außerdem wurde die Möglichkeit der individuellen Farbeinstellung als Ausdruck der Freiheit des Individuums verkauft - über Geschmack läßt sich bekanntlich nicht streiten. Im Gegensatz zu dieser liberalen Einstellung gegenüber neuen Technologien stellte der >Durchschnittseuropäer` erheblich höhere Qualitätsanforderungen. Aus zahlreichen Gesprächen mit europäischen Fernsehingenieuren geht hervor, daß eine Einführung des Farbfernsehens in Europa nach der 1954 erreichten Qualitätsstufe nicht denkbar gewesen wäre ${ }^{176}$. In diesem Punkt wird ein Aspekt dessen deutlich, was später unter dem Stichwort "nationale Technikstile« zu diskutieren sein wird.

Ein weiteres Argument, das aus amerikanischer Perspektive für die frühe Einführung von »noch nicht perfekten« Technologien sprach, war im Falle des Farbfernsehens die Belebung des »Dritten Sektors« (Dienstleistungen) der Wirtschaft, d.h. des Reparaturgeschäftes. Für die etwas unkritischere

175 „Da in einem stetigen Fortschritt die Qualität der Studiosignale verbessert und die Verzerrung der Übertragung herabgesetzt werden, ist die Bedienung dieser Regler immer seltener notwendig. Sie ermöglichen jedoch gewisse Änderungen der Farbbildwiedergabe, um dem individuellen Geschmack entgegenzukommen. Farbe ist, trotz allem, eine subjektive Empfindung, und nicht alle Beobachter sind mit dem zufrieden, was ein Wissenschaftler als optimale Farbwiedergabe bezeichnet«. WENTWORTH, Zusammenfassung der praktischen Erfahrungen mit dem Farbfernsehen in den Vereinigten Staaten, S. 244.

176 Diese Interpretation wird durch die Interviews des Autors mit Lang/Hausdörfer, Goussot, Melchior und In der Smitten bestätigt. 
Aufnahme des Farbfernsehens in den USA kann noch ein weiterer Punkt als mögliche Erklärung angeführt werden: die zeitlich eng beieinanderliegende Einführung des s/w- und des Farbfernsehens. In den führenden Fernsehländern Frankreich, England und Deutschland fällt die erste, wenn auch zaghafte Verbreitung des Schwarzweißfernsehens in die Phase der späten dreißiger Jahre. Bis zur ebenso zaghaften Verbreitung der Farbfernsehtechnologie vergingen nicht weniger als dreißig Jahre. Auch wenn man die Bedeutung des Zweiten Weltkrieges als folgenschwere Zäsur in den europäischen Ländern nicht unterschätzen darf, vergingen in den USA zwischen der Einführung des s/w-Fernsehens und der Einführung des Farbfernsehens nur zwölf Jahre. Während sich in den USA das »Faszinosum« Fernsehen für viele Menschen nicht in Schwarzweiß- und Farbtechnik spaltete, sondern eine mehr oder minder vage technologische Einheit bildete, ermöglichte die lange Zeitspanne zwischen SchwarzweiB- und Farbfernseheinführung in Europa ein Abebben der Faszination für das Phänomen Fernsehen bei gleichzeitigem Anstieg des kritischen Sehvermögens ${ }^{177}$. In einem Leitartikel der Fachzeitschrift Funk-Technik wird diese kulturanthropologische Differenz in der Aneignung der neuen Farbfernsehtechnologie beispielhaft herausgestellt:

Der europäische Fernsehteilnehmer ist gewohnt, an das empfangene Bild hohe Anforderungen zu stellen. Automatiken und Stabilisierungen im Empfänger geben ihm ein Fernsehbild, das auch beim Umschalten auf einen anderen Sender keiner Nachregelung bedarf. Es ist deshalb nur logisch, wenn der Fernsehteilnehmer bei dem kommenden Farbfernsehen als einer neuen Technik auch eine Stabilisierung des Farbtons als selbstverständlich voraussetzt. [...] Anders aber als damals [bei der Einfuibrung der s/w-Technik, A.d.V.], fasziniert heute nicht das Wunder Fernsehen als solches, sondern der ein qualitativ gutes und stabiles Bild gewohnte Fernsehteilnehmer will sich einen Eindruck von der Wirkung der Farbe machen. Wenn dann die Farbqualität zu wünschen übrig läBt, dann breitet sich nur zu leicht mit Windeseile die Meinung aus: ২Das Farbfernsehen steckt noch in den Kinderschuhen. Eine solche Meinung aber kann, wenn sie erst einmal weite Kreise erfaBt hat, den Start und den Durchbruch des Farbfernsehens erheblich erschwe$\mathrm{ren}^{178}$.

Die unterschiedlichen Erwartungshaltungen der amerikanischen und europäischen Konsumenten an die Qualität der Unterhaltungselektronik sind bis heute festzustellen. Auch Walter Bruch und Heide Riedel können als Zeugen dieser These angeführt werden. In ihrem PAL-Buch berichtet Bruch über die Erfahrungen mit dem Farbfernsehen in den USA im September 1953:

177 Eine Parallele kann hier zur Einführung des Hörrundfunks in Deutschland gezogen werden. Wahrend in den Anfangsjahren nach Beginn der öffentlichen Rundfunksendungen (1923) der "Apparat" als solcher im Mittelpunkt des Interesses stand, rückte im Laufe der Jahre das gesendete Programm in den Vordergrund der Wahrnehmung. Carsten Lenk beschreibt dieses Phänomen als "Domestizierung des Apparates" mit weitreichenden Folgen für die Sender- (Programmgestaltung) und Empfängerseite (geschlechterspezifische Aneignungsformen, Apparat wird zum Möbelstiuck etc.). LENK, Die Erscheinung des Rundfunks. Zur Domestizierung des Fernsehapparates siehe SPIGEL, Make Room For TV.

178 N.N., Zur europäischen Farbfernsehnorm, in: Funk-Technik 20 (1965). 5 S. 155. 
Als ich dann nach einem Besuch der Proben im Farbfernsehstudio der NBC die roten Haare von Heidi gesehen hatte, am Abend im Hotel dann die Sendung einschaltete und es mir mit keiner Einstellung gelang, die roten Haare so zu sehen, wie sie am Nachmittag auf den Monitoren im Studio geleuchtet hatten, da war mir klar: so konnte das für uns in Deutschland nicht eingeführt werden. Nur dann, so war jedenfalls meine Meinung, konnte uns Farbfernsehen im Heim in die angenehme Grundstimmung des Staunens, Wunderns und Behagens versetzen, wenn das Bild unverfälscht so ankam, wie es die Macher im Studio für uns gewählt hatten ${ }^{179}$.

Auch aus Frankreich waren ähnliche Stimmen zu vernehmen. So urteilte der Technische Generaldirektor der CSF, Roger Aubert, noch im Mai 1964, einer der wichtigsten Vorteile des SECAM-Systems sei "[...] de permettre de mettre entre les mains du public des récepteurs sans aucun réglage supplémentaire pour la couleur, ce qui me paraît une condition impérative pour une diffusion effective de la télévision en couleurs ${ }^{180}$. Allerdings sollte sich Aubert in seiner abschließenden Einschätzung über den Ausgang der europäischen Bemühungen um einen einheitlichen Farbfernsehstandard irren, als er sagte: "Ce qui est certain, c'est qu'un seul système sera choisi pour l'Europe. Lequel? Il est encore bien difficile de se prononcer ${ }^{181}$.

Das, was sich auf technischer Ebene im Anschluß an die Einführung des Farbfernsehens in den USA in Europa abspielte, kann mit den Worten des Funkschau-Redakteurs Otto Limann als die Suche nach einer "Automatikschaltung zum Einhalten der exakten Farbtöne« beschrieben werden ${ }^{182}$. An dieser Suche waren zahlreiche europäische Firmen beteiligt. Es muß darauf verzichtet werden, jede der entwickelten Alternativlösungen im Detail vorzustellen. Dies würde bedeuten, den Schwerpunkt der Darstellung auf die Diskussion technischer Details zu verlegen. Dabei wäre die Gefahr groß, jene übergeordnete Fragestellungen aus dem Auge zu verlieren, die im Mittelpunkt der historischen Analyse stehen sollten. Im Sinne einer historischen Innovationsforschung ist danach zu fragen, weshalb sich aus der Vielzahl technischer Alternativen gerade jene zwei Systeme (PAL und SECAM) als

179 Bruch, Riedel, PAL - Das Farbfernsehen, S. 65 f. Etwas weniger pathetisch formuliert es Bruch 1973 in einem Interview mit Funkschau-Chefredakteur Karl Tetzner: »Ich habe gesehen, da gibt es ein amerikanisches Farbfernsehen, ich habe es studiert, ich habe es bewundert, und ich bewundere es auch heute noch, aber ich habe auch gesehen, daß die Amerikaner anspruchslose Menschen in bezug auf die Farbe sind, sich mehr schlecht als recht mit dem Gebotenen zufrieden geben. Und es war mir völlig klar, daB wir niemals in der BRD auf diese Weise ein Farbfernsehen aufbauen könnten.« Interview Karl Tetzner mit Walter Bruch am 09.02.1973 in Ikking, 17 maschinegeschriebene Seiten, hier S.2f., in: Privatarchiv Prof. Tetzner, Icking bei München.

180 Roger AuBert, La télévision en couleurs - le problème européen, in: Bulletin de la Société Française des Électriciens (mai 1964) S. 11.

181 Ibid. S. 11.

182 Siehe Otto LimanN, Das Ziel der europäischen Farbfernsehnorm. Erste Farbfernsehtagung in der Öffentlichkeit, in: Funkschau 36 (1964) 14 S. 373. 
ernst zu nehmende europäische Konkurrenten des NTSC-Systems durchsetzen konnten? Welche technischen, industriellen, wirtschaftlichen und politischen Faktoren können als bedeutende Kontexte herausgearbeitet werden, die den Entscheidungsfindungsprozeß beeinflußt haben? Bevor diese Fragen ausführlich diskutiert werden, sollen die beiden Konkurrenzsysteme PAL und SECAM in knappen Zügen technisch charakterisiert werden.

\subsubsection{Das SECAM-System}

Wie bereits erwähnt, war das von Henri de France entwickelte und anfangs nach ihm benannte Farbfernsehsystem eines von drei in Frankreich entwikkelten Systemen. Laut eigenen Aussagen hatte Henri de France bereits 1949 mit Experimenten im Bereich des elektro-mechanischen Farbfernsehens begonnen ${ }^{183}$. Diese Experimente mündeten schließlich 1956 in das von ihm patentierte Farbfernsehsystem "Séquentiel Couleur à Mémoire«, später SECAM abgekürzt ${ }^{184}$. Was verbirgt sich hinter dieser Abkürzung? Henri de France' Ideen beruhten auf dem Gedanken, daß man ohne wesentliche Qualitätseinbuße die Vertikalauflösung eines Farbfernsehbildes verringern konnte, da die Fähigkeit des menschlichen Auges, Farbunterschiede bei sehr kleinen Strukturen (beispielsweise einen Bildpunkt) wahrzunehmen, beschränkt ist. De France setzte voraus, daß sich der zeitliche Verlauf der Farbinformation von einer Bildzeile zur zeitlich darauf folgenden Bildzeile nur unwesentlich ändert. Aus diesem Grunde, so seine Überlegung, bräuchten die für die Farbinformation erforderlichen Farbdifferenzsignale (B-Y) und (R-Y) nicht gleichzeitig (simultan) übertragen zu werden, sondern man könnte sie auch in jeweils zwei zeitlich aufeinander folgenden Zeilen nacheinander (sequentiell) übertragen. Im Empfänger setzt man dann die gesamte Farbinformation aus den in zwei aufeinanderfolgenden Zeilen übertragenen Komponenten wieder zusammen.

Dies war nur möglich, wenn es gelang, einen Speicher im Empfänger einzubauen, mit dem die Signalfolge einer Zeile für die Dauer einer Zeilenperiode gespeichert und mit der Signalfolge der nächsten Zeile gemeinsam verarbeitet werden konnte. Als Speichermedium benutzte Henri de France eine sogenannte Verzögerungsleitung (ligne à retard) ${ }^{185}$. Diese Verzögerungsleitung war das Herzstück des SECAM-Verfahrens, und sie wurde selbst von

183 Siehe die unveröffentlichen Memoiren von Henri de France, in: Archives du CHTV/INA, S. $67 \mathrm{f}$.

184 Die Patenterteilung in Frankreich erfolgte am 25. Mai 1956, in der Bundesrepublik als Patentschrift Nr. 951152 am 4. Oktober 1956.

185 Siehe das Telefunken-Fachbuch »Farb-Fernseh-Technik«, hrsg. von der Telefunken A.G., Ulm 1966, S. 105-111. 

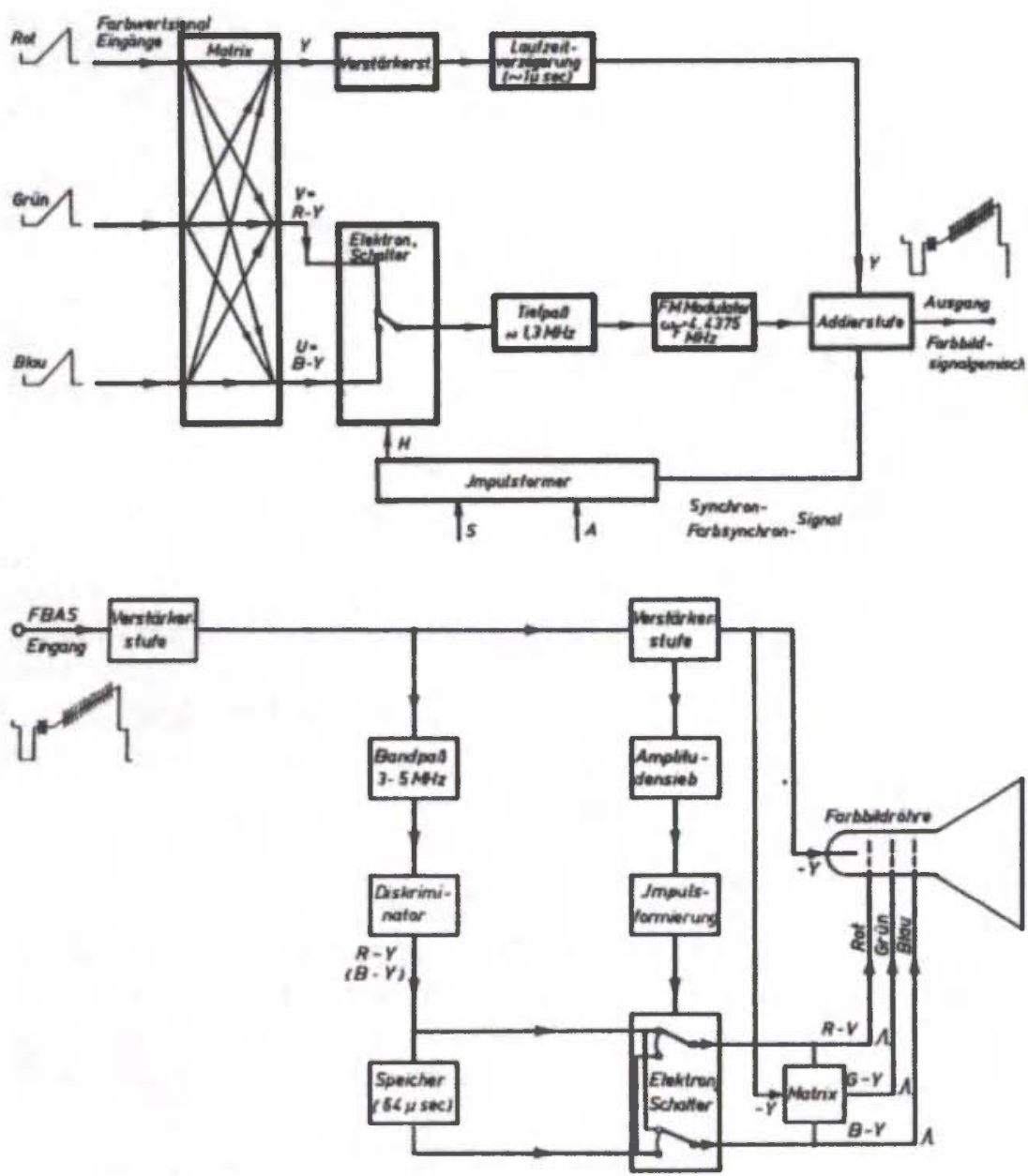

Abb. 6: Vereinfachtes Schema des SECAM-Verfahrens, aus: MAYER, Technik des Farbfernsehens, S. 231.

Kritikern de France' als große Erfindung gewürdigt ${ }^{186}$. Von diesen beiden Charakteristika - zeilensequentielle Übertragung der beiden Chrominanz-

186 Trotz der anfänglichen Schwierigkeiten, die man bei der Realisierung einer funktionsfähigen Verzögerungsleitung hatte, bemerkt Johannes Müller in einem rückblickenden Kommentar: „Dennoch wirkte der Impuls, den Henri de France mit seiner Idee der sequentiellen Utbertragung der beiden Farbkomponenten gegeben hat, in der Suche nach besseren Kodierungsverfahren weiter « - vor allem im PAL-System! MüLLER, Die intemationalen Bemühungen um eine europảische Farbfernsehnorm, S. 235. Auch Walter Bruch bescheinigte Henri de France, mit der Verzögerungsleitung eine »geniale Idee« gehabt zu haben, allerdings nicht, ohne diese Genialitằt im nächsten Satz ein wenig zu relativieren: „Ein guter Vorschlag, aber seine Realisation führte zu anderen Schwierigkeiten«. BRUCH, RIEDEL, PAL - Das Farbfernsehen, S. 65. 
komponenten und die Notwendigkeit ihrer Verzögerung um eine Zeile im Decoder des Empfängers - erhielt das Henri de France-System den Namen SECAM (Séquentiel Couleur à Mémoire) ${ }^{187}$. Pierre Braillard beschreibt die Funktion der Verzögerungsleitung im SECAM-System wie folgt: »Jetzt kann der Empfänger die ganze Zeile mit den drei Grundfarben bilden: dem Rot, das er im Augenblick empfängt, dem Blau, das er zum Zweiten Mal benutzt, und dem Grün, das er aus der Differenz berechnet « ${ }^{188}$.

Worin lag der Vorteil dieses Übertragungssystems? Wie gesehen, mußten beim NTSC-System beide Farbdifferenzsignale gleichzeitig übertragen werden, wofür die aufwendige Quadratur-Amplitudenmodulation angewendet wurde. Da das SECAM-System die beiden Komponenten der Farbinformation nacheinander übertrug, war keine Quadratur-Modulation notwendig - die Anfälligkeit des Farbhilfsträgers gegenüber Phasenverschiebungen (das große Problem des NTSC-Systems) fiel somit fort. Die Modulationsart der Farbhilfsträger konnte demnach frei gewählt werden. In seiner ersten Patentanmeldung verwendete das System zur Übertragung der Farbdifferenzsignale die einfache Amplitudenmodulation. Erst im Laufe weiterer Entwicklungen am System, die hauptsächlich von Mitarbeitern de France' bei der CFT geleistet wurden, meldete man im Oktober 1960 ein neues SECAM-Patent mit Frequenzmodulation der Farbdifferenzsignale an ${ }^{189}$. Der Übergang zur Frequenzmodulation bedeutete, daß das SECAM-System gegenüber Phasenfehlern »praktisch unempfindlich« war. Das zentrale Problem des NTSC-Systems war - zumindest theoretisch - auf diese Weise behoben ${ }^{190}$.

Allerdings stellte die Realisierung einer funktionsfähigen Verzögerungsleitung für die Mannschaft um Henri de France in der Anfangszeit ein großes Problem dar. Die elektrische Signalübertragung forderte eine sehr exakt berechnete Laufzeit des Signals, also der Dauer, die ein Signal für das Durch-

187 In seinen Memoiren berichtet Henri de France darüber, daß es eigentlich SEQUAM hätte heißen müssen, aber: »[...] alors SEQUAM, c'était pas très joli, alors on a dit: ça ne sonne pas bien, on va mettre SECAM, C'est resté comme ça.« Henri DE France, Mémoires, in: Archives du CHTV, S. 68.

188 Pietre Braillard, Die Geheimnisse des SECAM-Systems, in: Europa 16 (1965) 4 S. 50.

189 Das Patent trägt den Namen »Perfectionnements aux dispositifs de transmission utilisant une onde modulée notamment en fréquence«. Es wurde am 29. Oktober 1960 mit der Patentnummer 1278157 von Henri de France und Gérard Melchior angemeldet. Für eine Auflistung der wichtigsten Patentanmeldungen im Bereich der SECAM-Empfängertechnik siehe Brevets concernant le récepteur SECAM, in: Archives du MAE, Affaires Économiques et Financières, Affaires Générales, Nr. 922, dossier »SECAM en Hongrie«, 8 maschinegeschriebene Seiten. In diesem Dokument werden systematisch alle Anmeldedaten in ausländischen Patentämtern aufgeführt.

190 Eine ausführliche Beschreibung des SECAM-Systems kurz nach dem Wechsel von der Amplituden- zur Frequenzmodulation der Farbhilfsträger beschreiben die beiden Mitarbeiter von Henri de France, Pierre Cassagne und Marc Sauvanet in ihrem Aufsatz "Le Système de télévision en couleurs SECAM, comparaison avec le système NTSC, in: Annales de radiólectricite 63 (1961) S. 109-121. 
Abb. 7: Verzögerungsleitung aus Metall, aus: IndustrieAusstellung, Berlin 1966, S. 5, aus: Archives du CHTV/NA, Nachlaß Michel Dubail.

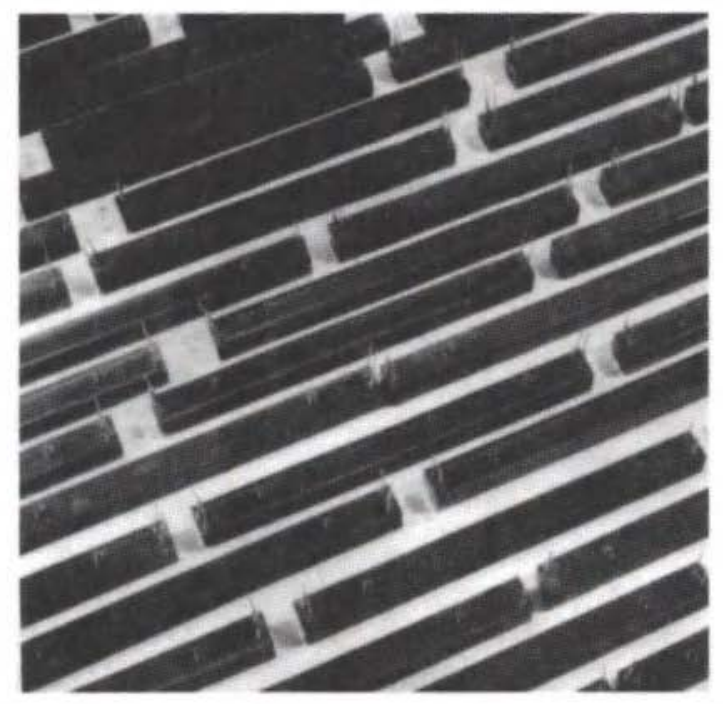

laufen einer Fernsehbildzeile benötigte. Nimmt man im Falle der europäischen Normen eine Zeilenzahl von 625 Zeilen und eine Bildfrequenz (f B) von $25 \mathrm{~Hz}$ an, ergibt sich eine Zeilenfrequenz (f H) von $625 \times 25=15625$ Hz. Daraus ergibt sich für die Dauer einer Bildzeile folgende Gleichung: TH $=1 / \mathrm{fH}=64 \mu \mathrm{s}$.

Die erste Verzögerungsleitung, welche Henri de France zur Speicherung eines Farbdifferenzsignals benutzte, war ein 64 Meter langes Koaxialkabel von $8 \mathrm{~mm}$ Durchmesser, in dem ein Videosignal (Ausbreitungsgeschwindigkeit von $300000 \mathrm{~km} / \mathrm{s}$ ) eine Strecke von einem Meter pro Mikrosekunde zurücklegte. Marc Chauvierre beschrieb die Schwierigkeit, mit der eine solche Konstruktion verbunden war, auf recht anschauliche Weise:

Seulement, les 64 mètres d'un câble qui fait environ 8 millimètres de diamètre tiennent beaucoup de place, méme enroulés en spirale. Si j'ai bonne mémoire, cela representait un coffre cubique d'environ cinquante centimètre de côté; disposé sous le récepteur, et ne parlons pas du prix de l'ensemble. Autrement dit, avec une ligne de retard de ce type, le système SECAM n'avait aucun avenir commercial et il fallait trouver autre chose ${ }^{191}$.

Eine Lösung dieses Problems bot die Idee, das elektrische Signal in eine Schallwelle umzuwandeln, deren Ausbreitung wesentlich langsamer war dementsprechend kleiner wurden die Abmessungen des Leitungskörpers. Am Ende des Leiters, man benutzte hierzu ein Metallstück von ca. 20 Zentimetern Länge, wurde die Schallwelle mittels eines Signalwandlers (als piezoelektrisches Element wurde meist ein Barium-Titanat-Wandler benutzt) wieder in das elekırische Ausgangssignal umgewandelt. Die Ultraschallfrequenz 
der akustischen Welle machte diese für den Fernsehzuschauer unhörbar. Da sie zudem aus Metall gefertigt wurde, konnte sie relativ kostengünstig in Serie produziert werden. Allerdings sollte sie schon bald durch Verzögerungsleitungen aus Glas ersetzt werden, da die Temperaturveränderungen im Fernsehempfänger das Metall in seiner Leitfähigkeit beeinflußten ${ }^{192}$.

Von der ursprünglich patentierten Idee bis zur Konstruktion eines in Serie produzierbaren SECAM-Empfängers sollten aber fast zehn Jahre vergehen. Dem ursprünglichen »Henri-de-France«-System mit amplitudenmoduliertem Farbhilfsträger (SECAM I) folgte 1961 das SECAM II-System mit frequenzmodulierten Farbdifferenzsignalen. Die Frequenzmodulation machte zwar gegen Phasenfehler unempfindlich, aber da sich im Gegensatz zur Amplitudenmodulation der Farbträger nicht unterdrücken läßt, konnte es zu stark störenden Farbträgermustern auf dem Bildschirm kommen, wenn man Farbsen-

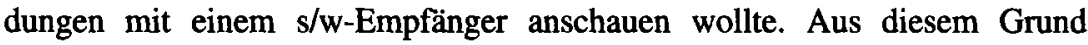
mußte der Farbträger im Coder des Senders abgeschwächt werden, was wiederum zu einer größeren Störempfindlichkeit des frequenzmodulierten Signals führen konnte (sogenannte Rauschstörungen) ${ }^{193}$. Schließlich wurden beim SECAM III-System für die Modulation der beiden Chrominanzkomponenten zwei unterschiedliche Trägerfrequenzen verwendet, um die Störungen des kompatiblen s/w-Empfangs durch den Farbträger weiter zu verringern ${ }^{194}$. Letztendlich führte man 1967 in Frankreich und der UdSSR das sogenannte »SECAM IIIb optimalisé« ein.

Diese ständige, schrittweise Modifikation des SECAM-Systems hat nicht gerade dazu beigetragen, das Image des Systems - vor allem bei deutschen Ingenieuren $-z \mathrm{u}$ fördern. Besonders deutlich tritt diese Kritik in einer Notiz von Walter Bruch an seinen Vorgesetzten Prof. Werner Nestel vom 6. September 1967 zutage. Hier bekannte Bruch:

Wie Sie wissen, bin ich ja nach meinen letzten Experimenten nicht mehr in der Lage, so viel gegen SECAM zu sagen. [...] Trotzdem bin ich nach wie vor nach allen Experimenten $100 \%$ ge-

192 Temperaturkonstante Glasverzögerungsleitungen waren bereits während des Zweiten Weltkrieges konzipiert worden. Dr. Fritz Kruse entwickelte 1942 bei Telefunken ein Spezialglas, bei dem das Signal proportional zur Erwärmung des Glases schneller lief. Allerdings war Anfang der sechziger Jahre nur die US-amerikanische Firma Corning Glass in der Lage, diese Ultraschall-Glasverzögerungsleitungen herzustellen. Siehe BRUCH, RIEDEL, PAL - Das Farbfernsehen, S. 67f.; N.N., Nouvelle ligne à retard pour le SECAM, in: Télévision (1963) 133 S. 127; CASSAGNe, SAUVANET, Le Système de télévision en couleurs SECAM.

193 Um dies zu erreichen, wendete man die sogenannte Pre-Emphasis-Technik an. Sie besteht darin, daß das aufzumodulierende Signal im Bereich seiner höheren Frequenzen angehoben wird. Die damit gegebene Anhebung der Signale im Bereich höherer Modulationsfrequenzen wird im Empfänger nach der Demodulation rückgängig gemacht. Siehe Telefunken AG (Hg.), Farb-Fernseh-Technik, S. $106 \mathrm{f}$.

194 Jacques FAGOT, Application de la mudulation de fréquence à la transmission du signal de chrominance de la télévision en couleur. Étude de la modulation SECAM, comparaison avec le NTSC, in: Annales de radioélectricité (janvier 1963) S. 3-15. 
Abb. 8: Henri de France, aus: Archiv des Deutschen Museums München, Nachlaß Walter. Bruch, Signatur $N L$ I0I, Nr. 123.

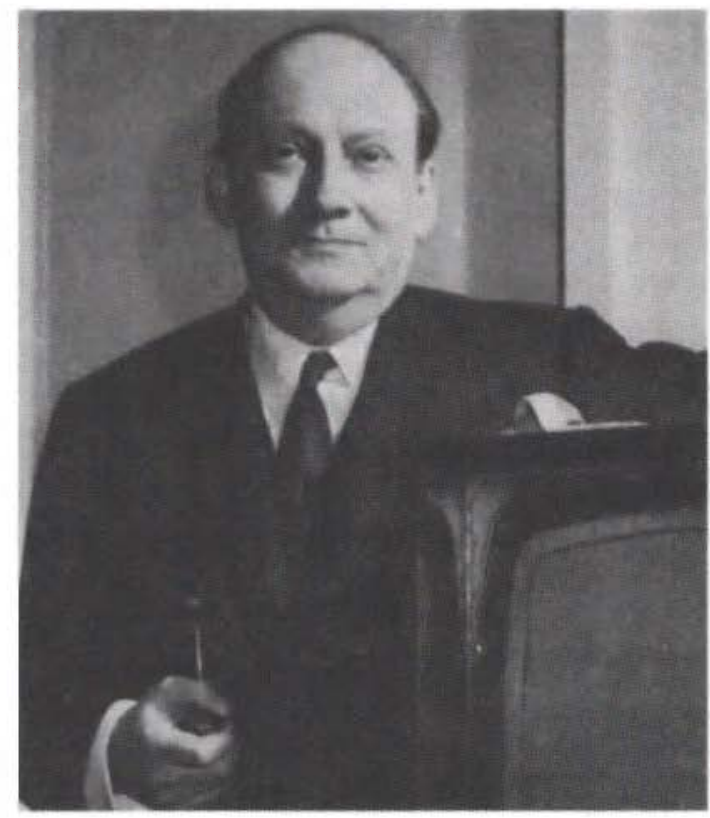

gen SECAM. Aber nur aus einem einzigen Grunde, weil SECAM sich so schwer realisieren läßt. [...] Leider läBt sich nicht in Worten ausdrücken, wie unsympathisch mir und all meinen Ingenieuren SECAM ist, weil es letzten Endes erbastelt wurde und nicht logisch enstand, daher sich mathematisch auch nicht exakt ausdrüicken läßt, im Gegensatz zu PAL, das analytisch sehr einfach berechnet werden $\operatorname{kann}^{195}$.

Wie "logisch« bzw. »wenig erbastelt« das PAL-System war, wird noch zu zeigen sein. Tatsächlich ist der Prozeß der »Systemverfeinerung « des SECAM-Systems vor allem als Reaktion auf den durch den neuen Konkurrenten PAL erzeugten Qualitätsdruck zu verstehen. Seit Ende des Jahres 1962 entwickelte sich zwischen beiden Systemen ein Konkurrenzkampf, der sich nicht zuletzt in immer weiteren technischen Verfeinerungen der beiden Systeme niederschlug. Die schrittweise Verbesserung der Systeme - also >Bastelei im Sinne experimenteller Versuche - läßt sich dabei genau so gut auf deutscher wie auf französischer Seite konstatieren.

Allerdings wurde dem SECAM-Erfinder Henri de France auch in Frankreich eher das Attribut eines »bricoleur de génie« als das eines systematischen Entwicklungsingenieurs zugestanden. Diese versteckt pejorative Charakterisierung der Fähigkeiten de France' aus den Reihen der französischen Kollegen ist mit hoher Wahrscheinlichkeit dem französischen Elitesystem

195 Notiz von Walter Bruch an Werner Nestel, »Vergleich PALSECAM«, Hannover, 6.09.1967, 2 Seiten, in: Telefunken-Archiv, Deutsches Technikmuseum Berlin, Signatur GS 1648. 
universitärer Ingenieurausbildung zu verdanken. Aus den Mündern erlesener »Polytechniciens « sollte die Bezeichnung »bricoleur de génie « nicht als Lob mißverstanden werden ${ }^{196}$. Fakt ist, daß das SECAM-System seine Entwicklung vom "genialen Einfall zum praktikablen System wesentlich den Ingenieuren der Compagnie Française de Télévision sowie den Ingenieuren der ORTF verdankte, die Henri de France großzügig zuarbeiteten. In den Hochzeiten der Systemauseinandersetzung zwischen PAL und SECAM waren es nicht weniger als hundert Ingenieure, Techniker und technische Zeichner, die bei der CFT mit der Entwicklung des SECAM-Systems beschäftigt waren. Henri de France sah sich selber eher in der Rolle des gedanken- oder impulsgebenden »Erfinders , der seine über Nacht ausgebrüteten Ideen am nächsten Morgen an seine Entwicklungsingenieure weitergab. Diese sollten dann überprüfen, ob sich aus seinen »Grübeleien« etwas Verwertbares machen ließ. So schreibt er in seinen Erinnerungen:

Je m'endors et je me dis: sapristi, il n'y a pas de solution à ce problème; alors je pose les données et je m'endors et le lendemain matin je dis: ah! Ben, c'est ça qu'il faut, on va essayer ça... ça ne réussit pas toujours hein, mais je dis: on va essayer ça, je vais trouver les amis ingénieurs, je dis: qu'est-ce que vous pensez si on essayait ça? Bon, bon, monsieur de France, on va essayer ça, oui, c'est peut-être à voir, bon... ${ }^{197}$.

Resultat dieser Arbeitsweise war, daß Henri de France nur an zwei von 25 Patenten namentlich beteiligt war, die zwischen 1956 und 1967 alleine im Bereich des SECAM-Empfängers angemeldet wurden ${ }^{198}$. In den Erinnerungen enger Mitarbeiter wird Henri de France eher als »Seher zukünftiger Entwicklungen« denn als Mann des konkreten experimentellen Arbeitens beschrieben. Pierre Cassagne, der als Entwicklungsingenieur der CFT ein wichtiger SECAM-Mitstreiter von de France war, schrieb:

Pour Henri de France, ce n'était pourtant là que des péripéties qu'il laissait à d'autres le soin de résoudre car son véritable combat était toujours un peu au-delà du présent. Je crois que ce fut une caractéristique constante de son action que d'avoir tourner sa réflexion vers le stade ultérieur de développement qu'il imaginait pour l'avernir. Il y avait là quelquefois de quoi dérouter ses collaborateurs souvent empêtrés dans les problèmes d'intendance si l'on peut appeler ainsi la poursuite d'une amélioration du rapport signal à bruit ou d'un gain de quelques francs sur le coût des circuits de réception ${ }^{199}$.

196 Siehe die Interviews mit Louis Goussot, Jacques Fagot und Yves Angel.

197 Henri de FranCE, Mémoires, in: Archives du CHTV/INA, S. 77.

198 Es waren die engen Mitarbeiter von de France bei der CFT wie G. Melchior, D. Brouard, M. Sauvanet, C. Ragot oder P. Cassagne, welche die meisten »brevets d'améliorations« des SECAM-Empfängers anmeldeten. Siehe Brevets concernant le récepteur SECAM, in: Archives du MAE, Affaires Economiques et Financières, Affaires Générales, Nr. 922, dossier »SECAM en Hongrie «, 8 maschinegeschriebene Seiten.

199 Pierte Cassagne, Au-dela du présent, in: Bulletin du Comité d'histoire de la télevision 14 (juin 1986), S. 28. Diese Nummer der Zeitschrift ist ganz der Erinnerung an Henri de France gewidmet und beinhaltet neben Erinnerungsfragmenten von Marc Chauvierre, Jacques Donnay, Michel Dubail, Armand Gaillard, André Goubert und André Debrie auch eine Zusammenstellung 
Allerdings hat Henri de France die >gute Naseく bei der Abschätzung zukünftiger technologischer Entwicklungen nur selten in profitable unternehmerische Aktivitäten umsetzen können. Wie er selbst zugibt, gehörte ein ausgefeilter Geschäftssinn nicht zu seinen hervorstechendsten Charakterzügen. Die Übernahme der Geschäftsführung von Radio Industries im Jahre 1940, die durch den Einmarsch deutscher Truppen in Frankreich bedingt war, »a très, très, très mal tourné « ${ }^{200}$. Letztlich war es wohl sein "génie de persuasion «, das Unternehmer, Banken und Politiker für seine Ideen zu gewinnen verstand. Bevor allerdings die Durchsetzungsstrategien auf nationaler und internationaler Ebene untersucht werden, muß mit dem PAL-System der dritte und letzte echte Konkurrent im Rennen um den potentiellen europäischen Farbfernsehstandard vorgestellt werden.

\subsubsection{Das PAL-System - „le troisième larron de la faible "201?}

\subsubsection{Die Entwicklungsgeschichte}

In der französischen Fachzeitschrift »Télévision« wurde das Erscheinen des PAL-Systems im Jahre 1963 mit folgenden Worten beschrieben: "Très inspiré du NTSC, peut-être un peu du SECAM, ce dernier-né de la technique TV en couleurs semble extrêmement intéressant, et pourrait bien devenir le troisième larron de la faible ${ }^{202}$. Wie sich herausstellte, sollte der unbekannte Verfasser dieser Zeilen recht behalten. Allerdings war, wie zu zeigen sein wird, der Entwickler des PAL-Systems, Telefunken-Ingenieur Walter Bruch, mehr als nur ein bißchen vom SECAM-System inspiriert. Nach der Einführung des NTSC-Systems in den USA im Jahre 1953 (und 1960 in Japan) wurde in mehreren europäischen Laboratorien an der Verbesserung des Systems gearbeitet. Auch in Deutschland beschäftigte man sich sowohl in der

einiger Passagen der unveröffentlichten »Memoiren« von Henri de France sowie einen Lebenslauf desselben.

200 Henri de France wurde Präsident von Radio Industries, weil der bisherige Präsident, sein Schwiegervater A. Vorms, als Jude die Geschäftsführung abgeben mußte. Siehe Henri DE France, Mémoires, in: Archives du CHTV/INA, S. 85f. Yves Angel geht sogar so weit, die finanziellen Pleiten als Charakteristikum de Francescher Unternehmungen auszumachen. "La caractéristique des krachs qui ont jalonné toute sa vie est qu'à chaque reprise de sa société moribonde par une société de bonne santé, Defrance retrouvait une place de direction qui ne tardait pas à devenir la place du patron, au moins de fait. Et quelques années plus tard, le processus recommencait, la proie grossissant à chaque fois. Il avait le génie de la persuasion pour emporter les affaires«. ANGel, Brève histoire des débuts de la tv française, S. 4.

201 Zitat aus N.N., Un troisième système de tv couleur: PAL, in: Télévision 137 (1963) S. 238. Im Neuen Testament werden die beiden Personen, die neben Jesus gekreuzigt wurden, als »larrons« (Schächer oder Mörder) bezeichnet. Der Ausdruck »le troisième larron « bedeutet im übertragenen Sinne »der Dritte im Bundeж.

202 Ibid. 
Rundfunkindustrie als auch in den Laboratorien der Landesrundfunkanstalten bzw. im zentralen Entwicklungslabor der ARD, dem Institut für Rundfunktechnik (IRT) in München, mit dem Farbfernsehen. Auch das Forschungslabor der Post, das Fernmeldetechnische Zentralamt (FTZ) in Darmstadt, machte ab Mitte der fünfziger Jahre Untersuchungen zum Farbfernsehen. Primär war diese Beschäftigung nicht mit dem Ziel verbunden, technische Verbesserungen des NTSC-Systems zu realisieren, sondern im Vordergrund stand das Bedürfnis, sich mit der für Deutschland noch in weiter Ferne liegendenden Technik - zumindest was ihre öffentliche Einführung in Form von Farbfernsehsendungen anbelangte - aus wissenschaftlich-technischer Perspektive vertraut zu machen. Auch die deutsche Rundfunkindustrie betrachtete das Farbfernsehen bis Anfang der 1960er Jahre eher als theoretisches Aktionsfeld, da das Geschäft mit dem Schwarzweißfernsehen erst seit Ende der fünfziger Jahre einen realen Aufschwung erlebte. Eine ernsthafte Auseinandersetzung mit dem Thema Farbe setzte - zumindest für die meisten Firmen des Zentralverbandes der Deutschen Elektroindustrie (ZVEI) erst zu dem Zeitpunkt ein, als eine erste Sättigungskurve im s/w-Geschäft abzusehen war, was erst 1964 der Fall war ${ }^{203}$.

Dennoch gab es vereinzelt Bemühungen, sich dem Thema Farbfernsehen intensiver zu widmen. So auch bei der Firma Telefunken, die durch ihre intensiven Kontakte zur französischen CSF schon früh mit dem Thema Farbfernsehen konfrontiert wurde. Bereits 1958 begann man auf Veranlassung des Direktors der Forschungsabteilung, des Vorstandsmitglieds Prof. Werner Nestel, mit der Untersuchung des NTSC-Systems in seiner an die europäische Zeilennorm und Bildfrequenz angepaßten Variante. Intensiver wurden die Beschäftigungen mit dem Farbfernsehen ab Dezember 1958. Bei einem der in regelmäßigen Abständen stattfindenden Besuche von Entwicklungsingenieuren von Telefunken bei ihren französischen Partnern der CSF in Paris führte man Bruch und Nestel das mittlerweile SECAM getaufte Farbfernsehsystem vor. Nach Deutschland zurückgekehrt berichtete Bruch auf der Sitzung der Funkbetriebskommission der ARD über das SECAM-Verfahren, wo es großes Interesse erzeugte. Man beschloß, »das Verfahren zu prüfen und in die Überlegungen für ein zukünftiges Farbfernsehen einzubezie-

203 Siehe Gespräch Bruch bei von Bismarck (Intendant des WDR) in Köln, Hannover, 3.7. 1964, in: Archiv des Deutschen Museums München, NachlaB Walter Bruch, Signatur NL 101, Nr. 202. Dort heiBt es: »Herr Meyer als Vorsitzender der Fachabteilung 14 erklärte, daB die Industrie in der Fernsehproduktion eine Durststrecke bekomme und unbedingt 1967 Farbfernsehen brauche. Bismarck wollte als Einfuihrungstermin lieber 1968, weil für den Rundfunk die Saison erst um die Weihnachtszeit beginne; dies wurde vom ZVEI strikt abgelehnt, da die Saison mit der Funkausstellung im Herbst beginnen muß«. 
hen $\aleph^{204}$. Als Prüfer wurden Walter Bruch und Prof. Richard Theile vom Institut für Rundfunktechnik (IRT) in München benannt. Bereits am 23. Dezember folgte aus Paris die positive Antwort, daß man dem IRT einen SECAM-Coder und -Decoder zwecks experimenteller Untersuchungen des Systems zur Verfügung stellen werde ${ }^{205}$.

Schon beim nächsten Besuch von Richard Theile und Walter Bruch in Paris im Januar 1959 wurde den beiden deutlich gemacht, daß die freundliche Atmosphäre, die den beiden während ihres Aufenthaltes entgegengebracht wurde, rundfunkpolitischen Hintergedanken zu verdanken war: Die Franzosen erhofften sich eine Unterstützung des SECAM-Systems auf der XI. CCIR-Vollversammlung in Los Angeles. Dies war aber für Bruch und Nestel nur denkbar, wenn sich die Franzosen auf den 625-Zeilenstandard für das Farbfernsehen in den Bändern IV und V zu bewegten. Da sich diese Wende im französischen Denken in Los Angeles andeutete, kam auch die deutsche Delegation mit einer günstigen Beurteilung des SECAM-Systems aus Los Angeles zurück. Im April 1960 legten die Ingenieure des IRT dann die ersten eigenen Untersuchungsergebnisse des SECAM-Systems vor. In Kurzform lautete »Ergebnis Erprobung IRT« wie folgt: »SECAM in seiner Leistungsfähigkeit gleich NTSC, aber verringerte Anforderungen an Sende- und Richtfunkgeräte ${ }^{206}$.

Dieses positive Gutachten aus München führte dazu, daß die Verantwortlichen der CSF einen offiziellen Antrag um Unterstützung des SECAM-Systems in Deutschland an Telefunken richtete. In den Notizen Walter Bruchs liest man:

CSF sprach den Wunsch aus, Telefunken möge sich voll und ganz gegenüber den deutschen Behörden für ihren Vorschlag einsetzen. Versteckte Drohung einer Zusammenarbeit mit Siemens. Zusage eines Briefes von Telefunken an CSF mit Erklärung des prinzipiellen Interesses, aber der Notwendigkeit eigener empfängerseitiger Versuche mit CSF-Apparatur. CSF braucht einen solchen Brief gegenüber den französischen Behörden. Der Brief verpflichtet uns in keiner Weise ${ }^{207}$.

Am 10. Mai 1960 erhielten die Verantwortlichen der CSF in Paris einen Brief in vorstehendem Sinne und diese bestätigten ihrerseits die Bereitschaft zu intensiver Zusammenarbeit. Da das SECAM-System nun auch Aufnahme in die Testversuche des Gerber-Ausschusses fand und somit in die internationale Diskussion im Rahmen der CCIR eingebunden war, bot die CSF ihrem deutschen Partner im Dezember einen »Pionier-Vertrag" an, der Telefunken

204 Siehe die chronologische Auflistung der Telefunken-CSF/CFT-Kontakte zwischen 1958 und 1967, 28 maschinegeschriebene Seiten, hier S. 2, wahrscheinlich von Walter Bruch zusammengestellt, in: Bruch-NachlaB im Archiv des Deutschen Museums München, Signatur NL 101, Nr. 202. Im folgenden zitiert als »Telefunken-CSF/CFT-Kontakte«.

205 Ibid. S. 2.

206 Ibid. S. 4.

207 Ibid. 
eine bevorrechtigte Position bei der Vergabe der CSF-Patente auf dem Farbfernsehsektor garantieren sollte. Außerdem wurde erwogen, auf der nächsten CCIR-Zwischentagung in Cannes einen Unterausschuß zum Vergleich des NTSC-Systems mit dem SECAM-System zu gründen. Beide Initiativen der Franzosen fanden in Hannover positive Reaktionen, doch als am 19. Juni 1960 ein erster Entwurf des »Pionier-Vertrages« eintraf, stellten sich die möglichen Nachteile dieses Abkommens größer als die potentiellen Vorteile heraus. $\mathrm{Zu}$ den Vertragsklauseln heißt es in den Aufzeichnungen von Walter Bruch:

Beschäftigung mit konkurrierenden Systemen untersagt (einschlieBlich Entwicklungsaufgaben für andere); Zwang der Werbung für SECAM nicht nur in Deutschland sondern international; Kündigungsmöglichkeiten für CFT, wenn Telefunken nach ihrer Ansicht nicht genug tut; fast keine Rechte für eventuelle eigene Erfindungen an SECAM; die für die Vorführungen notwendigen nicht spezifischen Anlagen auf eigene Kosten herzustellen. Gegenleistung: meistbegünstigte, nicht ausschließliche, später festzulegende Schutzrechts- und Nachbaulizenz, Beteiligung von anderen in Deutschland mit 10\%, aus Europa mit $5 \%$.

Obschon sich die Verhandlung über den »Pionier-Vertrag« zwischen Telefunken und der CSF/CFT über anderthalb Jahre hinzogen, kam es letztlich nicht zu einem VertragsabschluB. Zwar bedeutete dies nicht, daB Telefunken seine Förderung des SECAM-Systems gänzlich einstellte. Im Gegenteil. Durch die Unterstützung des SECAM-Systems »in Form wohlwollender Neutralität $\kappa^{209}$ durch Telefunken bei der CCIR Zwischentagung in Bad Kreuznach vom 13. bis 29. Juni 1962 gelang es, den Vorsitzenden der Studiengruppe XI, den Schweden Erik Esping, von der Notwendigkeit weiterer Systemuntersuchungen zu überzeugen und eine endgültige Entscheidung über die Empfehlung eines europäischen Farbfernsehstandards aufzuschieben $^{210}$.

Die wwohlwollende Neutralität« Telefunkens war aber nicht so uneigennützig, wie dies auf den ersten Blick scheinen mag. Zum einen erhoffte man sich bei Telefunken, daB »wenn sich die deutsche Industrie mit der SECAMFrage beschäftigt [...], dann sollte man uns in Deutschland die Führung in dieser Angelegenheit überlassen ${ }^{211}$. Zum anderen waren zu diesem Zeitpunkt Walter Bruchs eigene Forschungsarbeiten an einem verbesserten »NTSC/SECAM-System« schon so weit fortgeschritten, daß sie auf dem Zweiten Internationalen Farbfernsehsymposium in Montreux im Mai 1962

\section{Ibid. S. 7.}

209 In den Notizen von Walter Bruch vom 18.04.1962 heißt es: „Telefunken bietet weiterhin Unterstiutzung in Form >wohlwollender Neutralität` an wie bisher, dabei aber weitere eigene Labor-Beschäftigung mit SECAM bzw. einer Kombination SECAM/NTSC «, in: Telefunken-CSF/ CFT-Kontakte, S. 12.

210 Siehe Hans RiNDFLEISCH, Zwischentagung des CCIR in Bad Kreuznach in der Zeit vom 13. bis 29. Juni 1962, in: Rundfunktechnische Mitteilungen 6 (1962) S. 219.

211 Telefunken-CSF/CFT-Kontakte, S. 15. 
vorgetragen werden konnten. Ein Teilnehmer der Tagung schrieb, die Vorführungen des SECAM-Systems durch Ingenieure der CFT sowie die durch Walter Bruch präsentierten Verbesserungen des NTSC-Systems hätten gezeigt, »daB Möglichkeiten bestehen, die entscheidende Problematik des NTSC-Verfahrens, die Empfindlichkeit gegen differentielle Phasenverzerrungen, weitgehend zu lösen, so daß der sonst übliche `Farbtonknopf` beim Empfänger entfallen kann $\kappa^{212}$. Bruch behauptet in seinen Notizen sogar, er habe diesen Vortrag in Montreux nur gehalten, "damit die CFT in der Diskussion auf Verbesserungen von SECAM eingehen kann $\aleph^{213}$. Dies muß, wie zu zeigen sein wird, aus mehreren Gründen bezweifelt werden. Den Beginn seiner eigenen Entwicklungen im Sinne einer Kombination der beiden Systeme NTSC und SECAM datiert Bruch in internen Notizen mit dem 7. Juli 1961. Dort heißt es: "Nach Einstellung der Arbeiten an SECAM einen Gedanken in einem provisorischen Aufbau verwirklicht, der zum Erfolg geführt hat. $[\ldots]$ in zeitlicher Folge das Übertragungssystem so zu durchlaufen, daß hintereinander entgegengesetzte Farbfehler entstehen und entweder in der Matrix oder auf elektrischem Wege oder durch das Auge durch optische Integration diese Fehler ausgemittelt werden $\aleph^{214}$.

Bereits im November 1961 gelang es Bruch bei einem »kurzen improvisierten Versuch schon erstaunlich gute Bilder« mit seiner $»$ Variante des SECAM-Sytems [...] mit Ultraschall-Verzögerungsleitung mit Amplitudenmodulation bei unterdrücktem Träger « zu erzielen ${ }^{215}$. Wie mehrere Stellen bestätigen, fanden diese Experimente Bruchs mehr oder minder "schwarz" statt, denn Arbeiten im Bereich der Farbfernsehübertragungssysteme lagen außerhalb seines Forschungs- und Entwicklungsauftrages ${ }^{216}$. Dieser sah lediglich Aktivitäten im Bereich der Empfängerentwicklung vor, nicht aber im Bereich der Übertragungssysteme für Farbsignale. Was ihn dennoch bewog,

212 Siehe Friedrich von RaUtenfeld, Zweites internationales Fernsehsymposion in Montreux in der Zeit vom 30. April bis 4. Mai 1962, in: Rundfunktechnische Mitteilungen 6 (1962) S. $220 \mathrm{f}$.

213 Telefunken-CSF/CFT-Kontakte, S. 13.

214 Ibid. S. 7.

215 Ibid. S. 10.

216 In den Interviews mit Felix Herriger, Franz In der Smitten sowie mit Michael Hausdörffer und Heinwig Lang kommt zur Sprache, daB Walter Bruch ohne konkreten Entwicklungsauftrag an »seinem« System gebastelt habe. Und dies, obwohl ihm Ende 1962 wegen der Entscheidung des Telefunken-Vorstandes, die F\&E-Aufgaben zukdinftig auf den Bereich der GroBrechner zu konzentrieren, die Hälfte der ihm in Hannover unterstehenden Entwicklungsingenieure abgezogen worden waren. Bruch kommentierte diesen herben Schlag für ihn wie folgt: "So verblieben mir noch vier Ingenieure, ein Sachbearbeiter und eine Zeichnerin, dazu zwei Mechaniker und eine Fulle mir übertragener Entwicklungen für das SchwarzweiBfernsehen. Meine Absicht, zu einer anderen Firma überzuwechseln, ließ sich nicht durchführen, da mein Anstellungsvertrag eine Konkurrenzklausel enthielt, die mir zwei Jahre nach dem Ausscheiden eine Arbeit auf dem gleichen Arbeitsgebiet versagte «. BRUCH, RIEDEL, PAL - Das Farbfernsehen, S. 70. 
in diesem Feld aktiv zu werden, beschrieb Bruch in seinem PAL-Vermächtnis: "Bei den Untersuchungen an dem ersten SECAM waren mir bald die Mängel aufgefallen, die seiner Einführung als Einheitssystem für Europa im Wege standen. Das Vorpreschen der Franzosen hatte mir Mut gemacht, mich an eigenen Ideen experimentell zu versuchen«. Forschungen im Bereich des Farbfernsehens sollten - von dem SECAM-Auftrag abgesehen - »nur so weit [gehen] - nach den Wünschen der Geschäftsleitung -, wie es die in Hannover später zu produzierenden Farbfernsehempfänger betraf. Doch die mir als wichtig übertragene Arbeit an SECAM vorschiebend, gelang es mir, - mehr oder minder schwarz -, Entwicklungen an eigenen Systemen durchzufüh$\operatorname{ren}^{217} \ll$.

Wie Bruch in mehreren zeitgenössischen Darstellungen betonte, ging es ihm ursprünglich nicht darum, ein eigenes Farbfernsehübertragungsystem zu entwickeln, sondern die vorhandenen technischen Alternativen zu optimieren. In einem internen Telefunken-Papier aus dem Jahre 1970 hat Walter Bruch dem Leiter der Telefunken-Patentabteilung, Dr. Johannesson, seine Version zur Entstehungsgeschichte des PAL-Systems vorgelegt. In dem neunseitigen Vortrag mit dem Titel »Wie kam es zu PAL und zum alternierenden Burst?» erklärte Bruch:

Ich hatte nicht die Vermessenheit daran zu denken, daß ich das NTSC-System je vom Geber her ändern könnte. [...] Ich selbst habe damals nie die Absicht gehabt, ein neues System zu entwikkeln. Meine Gedanken gingen immer dahin, das NTSC-System im Empfängereingang so abzuwandeln, daß es fehlerlos durch den Empfänger geht. [...] Gesucht wurde von mir eine Beseitigung der Seitenbandfehler, nicht einer Beseitigung der Fehler der Grundphase. Damals war ich noch überzeugt, daß man das mit einem Knopf einstellen kann ${ }^{218}$.

Immer wieder betont Bruch in diesem Vortrag, daß ihn seine damaligen Vorgesetzten ständig darauf hingewiesen hätten, seine F\&E-Bemühungen ganz auf das Gebiet der Farbempfänger zu konzentrieren, weshalb er nie auf die Idee gekommen sei, seine frühen Ideen im Bereich der Farbsignalübertragung zu patentieren. Die Idee der sequentiellen Übertragung der Farbsignale, so Bruch, sei ihm bereits vor Bekanntwerden des »Henri de France«-Systems eingefallen.

Es fällt ein wenig schwer, diese retrospektive Darstellung Bruchs im Detail als "wahrheitsgetreu « zu akzeptieren, auch wenn nur indirekte Beweise als Gegendarstellung herangezogen werden können. So schreibt Bruch selber in seinem PAL-Buch, das Vorpreschen der Franzosen habe ihm Mut gemacht, sich »an eigenen Ideen experimentell zu versuchen ${ }^{219}$. Dieser Schil-

217 Ibid., S. 65.

218 Walter BRUCH, Wie kam es zu PAL und zum alternierenden Burst?, ein Vortrag vor dem Leiter der Patentabteilung, Dr. Johannesson, im Sommer 1970, in: Archiv des Deutschen Museums München, NachlaB Walter Bruch, Signatur NL 101, Nr. 178, 9 maschinegeschriebene Seiten.

219 BRUCH, RIEDEL, PAL - Das Farbfernsehen, S. 65. 


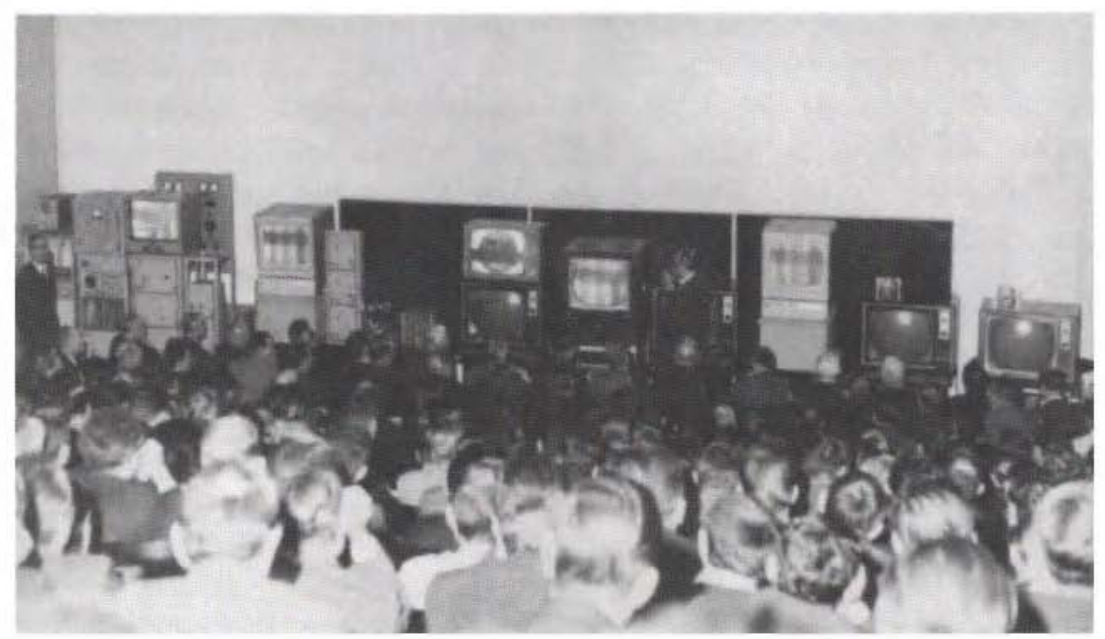

Abb. 9: Walter Bruch während eines PAL-Demonstrationsvortrages 1966 vor dem Verband Deutscher Elektroingenieure (VDE) und dem Verband der Postingenieure im Auditorium Maximum der Ingenieurhochschule Hannover, aus: Archiv des Deutschen Museums München, Nachlaß Walter Bruch, Signatur. NL 101, Nr. 259.

derung widerspricht er an anderer Stelle, wo es heißt, daß er sich an das »Henri de France $\ll-S y s t e m$, von dessen Existenz er 1956 in Paris erstmals in einem Vortrag von R. Cahen gehört habe, nur »nebelhaft« erinnert habe. Genau in dieses Jahr 1956 fallen aber Bruchs erste experimentelle Versuche im Bereich der sequentiellen Übertragung der Farbhilfsträger. Auch die Idee des alternierenden Bursts, also die Bildung des späteren PAL-Farbsignals durch die videofrequente Umpolung, habe er in Unwissenheit der Ideen von Loughlin entwickelt, heißt es in diesem Bericht ${ }^{220}$. An anderer Stelle gibt er aber zu, daß er, als er sein neues System erstmals auf dem Farbfernsehsymposium in Montreux im Mai 1962 vorgestellt habe, sogar ausdrücklich auf die Vorarbeiten von Loughlin hingewiesen habe ${ }^{221}$.

Diese Widersprüche scheinen auf den ersten Blick nicht von großer Wichtigkeit, sollten aber in der späteren Phase der PAL-Propagierung von entscheidender Bedeutung sein, als es um die patentrechtlichen Grundlagen der Lizenzforderungen ging, die in einem späteren Abschnitt geschildert werden. Es ist daher nicht erstaunlich, daß der erste Versuch von Bruch, sein Kombinationssystem von SECAM und NTSC im Jahre 1962 zu patentieren, vom deutschen Patentamt abgelehnt wurde.

$220 »$ Der Gedanke, die Punktkombination umzupolen, ein Gedanke, den vor mir schon Herr Loughlin gehabt hatte, dessen Arbeiten ich nicht kannte, und der von ganz anderen Gesichtspunkten ausging [...]«. BRUCH, Wie kam es zu PAL und zum alternierenden Burst, S. 6.

221 BRuCh, RiEdEL, PAL - Das Färbfernsehen, S. 66. 
Dieses als »Volks-PAL « oder »Simple-PAL « bezeichnete und am 29. September 1962 beim Deutschen Patentamt in München eingegangene "Farbfernsehübertragungssystem $«$, das Telefunken-intern die Bezeichnung »W 3807« trug, wurde am 17. April 1963 vom zuständigen Sachbearbeiter zurückgewiesen. In der Begründung von Dipl. Ing. Költer hieß es speziell zu Bruchs Idee der alternierenden Übertragung der Phasenlage des Farbvektors:

Bei dieser Sachlage erscheint es verhältnismäßig naheliegend, anstatt den Farbsynchronimpuls zu unterdrücken, diesen in der Phase um $180^{\circ}$ zu drehen. Dies ist ein in der Nachrichtentechnik bekanntes Prinzip [...] Wird der Anspruch 1 aufrechterhalten, so muB mit der Zurückweisung der Anmeldung mangels ausreichender Erfindungshöhe und mangels Ausschaltung von unbrauchbaren Lösungen (zeilenfrequente Umschaltung) gerechnet werden ${ }^{222}$.

Nach sechsjährigem Hin und Her mit der Patentbehörde wurde im Mai 1968 die als »PAL 1 « bezeichnete Patentanmeldung von Telefunken zurückgezogen, da - wie es in einem internen Telefunken-Schreiben heißt - »wegen der zweifelhaften Offenbarung aufgrund des Zwischenbescheides vom 21.4. 1967 die Aussicht [besteht], daß W 3084 versagt wird « ${ }^{223}$. Dieser Verzicht war jedoch für Telefunken unproblematisch, da der Schutzumfang von W 3084 in dem Patentantrag W 3935, dem später tatsächlich gewährten Patent, wiederholt wurde und somit den gleichen Schutzumfang bot. Erst das am 31.12.1962 angemeldete Patent »Ein Farbfernsehempfänger für ein farbgetreues NTSC-System « ${ }^{224}$, das später als »Standard-PAL «-Patent bezeichnet wurde, wurde von der Patentbehörde akzeptiert, da es die Mittelung der von Zeile zu Zeile ankommenden Signale nicht mehr durch das Auge vorsah, sondern dies nun elektronisch im Empfänger durch einen speziell entwickelten Decoder geschah ${ }^{225}$. Es hatte also mehrerer Anläufe bedurft, bis das PAL-System vor dem Deutschen Patentamt den Status »ausreichender Offenbarung « erhielt.

Auf Grund der Ungereimtheiten in den Selbstdarstellungen Bruchs sowie der komplizierten patentrechtlichen Lage fallt es schwer, Bruchs erfinderische Leistung objektiv einzuschätzen. Fest steht, daB Bruch - was auch in seinen frühen PAL-Publikationen zum Ausdruck kommt - anfangs nicht dar-

222 Spätere Autoren weisen mehrfach auf Loughlins 1951 patentierte Idee der alternierenden Übertragung der Phasenlage des Farbvektors hin. Siehe beispielsweise MülLER, Die internationalen Bemühungen um eine europäische Farbfernsehnorm; G. B. Townsend, PAL Programm, Organization. A First Look at all the Problems, in: The Royal Television Society Journal 12 (1968) 1 S. 2-11.

223 Bei W 3084 handelt es sich um den Patentantrag DAS 1.161.949 (PAL 1). Siehe Aktennotiz, F\&E, Hannover, 20.5.1968, in: Archiv des Deutschen Museums München, NachlaB Walter Bruch, Signatur NL 101, Nr. 175.

224 Die Telefunken-interne Bezeichnung lautete W 3935 (»Laufzeitdemodulator «).

225 Gerhard MAHLER, Entwicklung und Einführung von PAL, in: BTS - Broadcast Television Systems (Hg.): 25 Jahre PAL-Farbfernsehen in Deutschland, firmeninterne Publikation, 1992, S. 9. 
an dachte, seine Weiterentwicklung des NTSC-Systems als »neues System« zu propagieren. Auch wenn das - wie sein ehemaliger Mitarbeiter Gerhard Mahler betont -, was Bruch de facto in seinem Labor tat, eher auf die Entwicklung eines eigenen Systems hinauslief ${ }^{226}$. In einem Brief an den damaligen Vorsitzenden des Fachverbandes 14 des ZVEI, Herm Boom, bekannte Bruch Ende des Jahres 1964:

In jener ersten Zeit war ich noch keineswegs 100\% für PAL. PAL war nur eine der Möglichkeiten, die ich demonstriert habe, und Sie werden sich erinnern, daB ich auch das von mir entwikkelte SECAM-System vorgeführt habe und auBerdem NTSC. [...] Ich hätte auch - wenn ich mich nicht für ein eigenes System entschieden hätte - irgendwie Stellung bezogen, denn das ist so meine Art. Ich bin heute nicht sicher, ob diese Entscheidung nicht vielleicht SECAM gewesen wäre, aber allerdings ein SECAM-System, das stark gegen das heutige SECAM-System verändert gewesen wäre. Die wesentlichen Vorteile von SECAM - die SECAM ohne Zweifel hat sind verlorengegangen im Laufe der Entwicklung von SECAM I zu SECAM III. Ob sich ein SECAM-System hätte finden lassen, das alle unsere Wünsche befriedigt hätte, ist heute nicht sicher. Aber meine Meinung ist ganz eindeutig: SECAM III nie! ${ }^{227}$

Den bereits erwähnten Vorwurf von Walter Bruch an die Adresse von Henri de France, das SECAM-System sei »erbastelt« und nicht »logisch entwikkelt«, muß sich Bruch wohl oder übel selbst gefallen lassen. Der Weg vom "SECAM I zum »SECAM IIIb optimalisé« war nicht weniger wandlungsreich als der vom »Volks-PAL« über »Standard-PAL« bis zum »PAL-de-Luxe« - er war nur länger! Die Länge des Weges bzw. die Dauer des Optimierungsprozesses eines technischen Systems kann aber kein Indikator für die Qualität des letztlich erreichten technischen Artefakts sein.

\subsubsection{Die Technik des PAL-Systems}

Obschon Bruch in einem 1973 mit Funkschau-Chefredakteur Karl Tetzner geführten Interview seine Leistung als »Verkäufer« des PAL-Systems höher einschätzte als seine »Erfindertätigkeit ${ }^{228}$, müssen die grundlegenden technischen Charakteristika des PAL-Systems im folgenden kurz dargestellt werden. In knappen Worten umschrieb Johannes Müller, leitender Direktor des Forschungstechnischen Zentralamtes (FTZ) der Bundespost in Darmstadt, das PAL-System mit folgenden Worten:

\footnotetext{
226 Ibid. S. 6.

227 Bei dieser Quelle handelt es sich um den von Bruch auf Tonkassette diktierten und transkribierten Entwurf eines Briefes an Herrn Boom, der leider nicht datiert ist. Da im Brief aber Bezug auf die Entscheidung des ZVEI genommen wird, dessen Technische Kommission am 12.11.1964 seine Entscheidung bekanntgegeben hatte, das die deutsche Elektroindustrie das PAL-System als europäische Farbfernsehalternative unterstïtzen werde, ist eine Datierung Ende November 1964 wahrscheinlich. In: Archiv des Deutschen Museums, NachlaB Walter Bruch, Signatur NL 101, Nr. 202, 4 maschinegeschriebene Seiten mit handschriftlichen Korrekturen von Walter Bruch.

228 Siehe Interview Prof. Tetzner mit Walter Bruch, 09.02.1973 in Icking bei München, 17 maschinegeschriebene Seiten, hier S. 2, in: Privatarchiv Karl Tetzner.
} 
So versuchte Walter Bruch in der Abteilung für Grundlagenentwicklung bei der Firma Telefunken, die Vorteile der sequentiellen Übertragung der Farbkomponenten (SECAM) mit der bewährten Amplitudenmodulation mit unterdrücktem Träger (NTSC) zu kombinieren, um dadurch die Nachteile der schlechteren Kompatibilität des SECAM-Verfahrens zu umgehen. [...] Das sequentielle Prinzip wird im PAL-Verfahren aber nur insoweit angewendet, als nicht die einzelnen Farbdifferenzsignale, sondern nur die Phasenlage des aus beiden Farbdifferenzsignalen resultierenden Farbvektors zeilenweise im Wechsel übertragen wird ${ }^{229}$.

Im Unterschied zum SECAM-System zielt beim PAL-System das Prinzip der sequentiellen Übertragung der Farbdifferenzsignale nicht auf die Auswahl jeweils nur eines Farbdifferenzsignals für die Trägermodulation, sondern auf den periodischen Wechsel der Phasenlage eines der beiden stets gleichzeitig gesendeten Farbdifferenzsignale. Die im NTSC-Verfahren gewählte Amplituden-Quadraturmodulation wird also beibehalten. Der Unterschied besteht aber darin, daß jeweils von Zeile zu Zeile die Phasenlage eines der beiden Farbdifferenzsignale periodisch umgeschaltet wird - daher der Name PAL = Phase Alternation Line. Dadurch wechseln die durch Phasenverzerrungen hervorgerufenen Farbtonfehler entsprechend ihren Drehsinn (auf die komplexe Farbebene übertragen) und können sich bei geeigneter Empfangsart ausmitteln ${ }^{230}$.

Die eigentliche Neuheit des PAL-Systems war, die von Loughlin entwikkelte Idee der alternierenden Übertragung der Phasenlage mit Henri de France' Idee der zeilensequentiellen Speicherung der Farbinformation eines Farbdifferenzsignals zu kombinieren. Die Mittelung des Farbtonwertes wurde durch eine elektronische Schaltung im Empfänger realisiert (Standard-PAL). »Richtig ausgenutzt wird das PAL-Prinzip erst mit Hilfe der - aus der SECAM-Technik bekannten und dort für die wirtschaftliche Massenproduktion entwickelten - Ultraschall-Verzögerungsleitung«, so die Einschätzung des Direktors des IRT in München, Prof. Richard Theile ${ }^{231}$. Es war also die Entwicklung eines speziellen Decoders mit Laufzeitleitung, die das PAL-System zu einer echten Alternative des NTSC-Systems machte. Mittels dieses Decoders wurde die lang ersehnte Stabilisierung des Farbtons erreicht, die noch bei Phasenfehlern gelang, die etwa $15 \mathrm{mal}$ so groß sein konnten wie jene, die bei einem normalen NTSC-Empfänger zu starken Farbtonverzerrungen führten ${ }^{232}$.

Zentraler technischer Baustein des PAL-Systems war demnach die bereits beim SECAM-System vorgestellte Ultraschall-Verzögerungsleitung. Auch die Verzögerungsleitung wurde zum Streitobjekt zwischen der CFT und Telefun-

229 MÜLLER, Die internationalen Bemühungen um eine europäische Farbfernsehnorm, S. 236.

${ }^{230}$ Siehe Richard THEILE, Die Entwicklung der kompatiblen Farbfernsehtechnik unter besonderer Berücksichtigung der verschiedenen Vorschläge für die Übertragung der Farbart-Signale (Farbträgermodulation), in: Rundfunktechnische Mitteilungen 9 (1965) 5 S. 241-250, hier S. $248 \mathrm{f}$.

231 Ibid. S. 248.

232 M. Koubek, Zwanzig Jahre kompatibles Farbfernsehen. Der Stand der Farbfernsehtechnik Rückblick und Ausblick, in: Radio-Elektronikschau 16 (1966) 4 S. 210. 
ken. Bereits 1964 wurde über die mögliche Abhängigkeit des PAL-Systems vom SECAM-System debattiert. Dieser Streit zog sich bis 1968 hin, als zwischen beiden Parteien ein sogenannter »Nichtangriffs-Pakt" geschlossen wurde, auf den an späterer Stelle noch eingegangen wird. Tatsächlich kamen Walter Bruch und seine Mitarbeiter erstmals im Mai 1960 in den Besitz einer CFT-Verzögerungsleitung, als dem Telefunken-Labor die SECAM-Versuchsanlage vom IRT zu Versuchszwecken weitergeleitet worden $\operatorname{war}^{233}$. Bei dieser ersten Verzögerungsleitung handelte es sich um ein Videoverzögerungskabel mit einem ferromagnetischen Kern als Koaxialkabel von 32 Metern Länge. Das Kabel ermöglichte also zwei Mikrosekunden Verzögerung pro Meter $^{234}$ und war damit nur mehr halb so lang wie die ursprünglich von Henri de France benutzte Leitung. Wie bereits beschrieben, wäre das SECAM-System mit einer solchen Verzögerungsleitung nie als ernsthafte technische Alternative zum NTSC diskutiert worden. Erst die Entwicklung einer Ultraschall-Verzögerungsleitung löste dieses Problem. Interessant ist, daß Walter Bruch immer wieder darauf hingewiesen hat, daß er es gewesen sei, der den CFT-Ingenieur Pierre Cassagne auf die Möglichkeit einer solchen UltraschallVerzögerungsleitung hingewiesen habe. In seinem Vortrag vor dem Leiter der Patentabteilung Dr. Johannesson im Sommer 1970 hieß es diesbezüglich:

Ich möchte noch einmal ausdrücklich darauf hinweisen, daß ich Herrn Cassagne auf die Ultraschallverzögerungsleitung hingewiesen habe. Ich hatte ihn damals auf eine Leitung hingewiesen, die die Firma Mullard aus England aus Quecksilber herstellte. Ich habe selbst darauf hingewiesen, daß ich mit Mullard darüber Verbindungen geknüpft habe, die aber erfolglos waren, weil Mullard diese Leitungen nur für Radar-Zwecke gebaut hat. In der Zwischenzeit konnte sich SECAM [gemeint ist sicher die CFT, A.d.V.] eine Leitung von der Firma Quarz und Silice beschaffen und freundlicherweise beschaffte man für mich eine solche Leitung mit ${ }^{235}$.

In mehreren Quellen erwähnt Bruch, daß die Idee der Ultraschallverzögerung eines elektrischen Signals eine Erfindung des Hauses Telefunken während des Zweiten Weltkrieges gewesen sei. Er habe zusammen mit dem Erfinder, Herrn Dr. Kruse, Ultraschallverzögerungsleitungen aus Glas für das Gerät "Rehbock« gebaut ${ }^{236}$. Während Bruch in seinem PAL-Buch bezüglich der

\footnotetext{
233 Telefunken-CSF/CFT-Kontakte, S. 4.

234 Das Kabel stammte von der Hannover Firma Hackethal (heute Kabelmetall) und entspricht genau den Beschreibungen, die Marc Chauvierre überliefert hat. Ein wenig übertreibend erinnert sich Chauvierre: »La ligne à retard d'Henri de France était un monstre: elle était constituée par des centaines de mètres d'un câble coaxial enroulé sur lui-même dans une caisse aussi grosse que le récepteur «. Siehe Marc ChauvierRe, Un homme passionné, in: Bulletin du Comité d'histoire de la télévision 14 (juin 1986) S. 36. Siehe auch MAHLER, Entwicklung und Einführung von PAL, S. 6.

235 BRUCH, Wie kam es zu PAL und zum alternierenden Burst?, S. 5.

236 Dabei handelte es sich um die Versuchskonstruktion eines RadarmeBgerätes. Siehe BRUCH, RIEDEL, PAL - Das Farbfernsehen, S.67. Bruchs unkritische und wenig distanzierte Haltung zum Nazi-Regime und zum Zweiten Weltkrieg kommt deutlich in seinem Aufsatz »Peenemünde
} 
Idee, Ultraschallverzögerungsleitungen für den Einsatz beim Farbfernsehen vorzusehen, sagt, "wer zuerst auf die Idee kam, weiß ich nicht mehr«, heißt es in eben jenem Vortrag vor Dr. Johannesson:

Ich weiß noch ganz genau, daß Herr Anderson - heute Chef der RCA-Fernsehentwicklung und Fabrikation in Indianapolis - mit mir sehr ausfuihrlich über solche Leitungen diskutiert hat, weil ich einmal im Krieg - wohl als erster außer dem Erfinder Herm Dr. Kruse - mit Ultraschallverzögerungsleitungen für das Gerät >Rehbock` gearbeitet habe. Damals [Ende 1956, A.d.V.] kam mir der Gedanke auf, eine solche Verzögerungsleitung auch für die Fehlerkompensation für das Farbfernsehen zu verwenden. SECAM war damals noch nicht erfunden ${ }^{237}$.

Abgesehen von der Tatsache, daß das SECAM-System unter dem Namen »Henri-de-France«-System bereits existierte und Bruch selber im April 1956 in Paris von diesem System erfahren hatte, dauert es laut Angaben Bruchs seltsamerweise bis zum November 1961, bis er sich in Hannover mit seinem Kollegen Cassagne von der CFT über die Ultraschall-Verzögerungsleitung unterhalten haben will ${ }^{238}$. 1961 verfügte die CFT aber bereits über eine funktionierende Ultraschall-Verzögerungsleitung der Firma Quarz und Silice, wie aus einer Publikation der beiden CFT-Ingenieure Pierre Cassagne und Marc Sauvanet hervorgeht ${ }^{239}$.

Auch in diesem Falle fällt es schwer, die "Wirklichkeit« des Erfindungsbzw. Entwicklungsprozesses zu rekonstruieren. Zum einen sind die Aussagen Walter Bruchs widersprüchlich, zum anderen decken sie sich nicht mit der französischen Überlieferung ${ }^{240}$. Fest steht, daß eine patentrechtlich abgesicherte Unabhängigkeit des PAL-Systems vom SECAM-System gerade aufgrund der in beiden Systemen verwendeten Ultraschall-Verzögerungsleitung nie festgestellt werden konnte, da beide Seiten letztlich den Gang vor das $\mathrm{Pa}$ tentgericht scheuten und sich außergerichtlich im so genannten »Nichtangriffs-Pakt« einigten ${ }^{241}$.

1942: Die Anfänge des >Industriefernsehens $<$. Erinnerungen an einmalige Erlebnisse in der Pionierzeit des Fernsehens« zum Ausdruck. In: Funkschau 5 (1974) S. 142-146.

237 BRUCH, Wie kam es zu PAL und zum alternierenden Burst?, S. 4.

238 Ibid. S. $8 \mathrm{f}$.

239 Pierre Cassagne, Marc Sauvanet, Le système de télévision en couleurs SECAM en comparaison avec le système NTSC, in: Annales de Radioéléctricité 63 (1966) S. 115.

240 Siehe die Interviews mit Gérard Melchior, Michel Dubail und Louis Goussot.

241 Als der CFT-Direktor Henri Peyroles auf der CCIR-Konferenz in Wien 1965 öffentlich behauptete, PAL sei von SECAM-Patenten (Verzögerungsleitung) abhängig, bereitete man sich bei Telefunken auf eine Gegendarstellung vor. Patentanwalt Dr. Johannesson teilte dem Vorstandsmitglied und Leiter der Forschung, Prof. Werner Nestel, in einer Note vom 31. Mai 1965 mit, $\mathrm{daB}$ die Idee der Ultraschallverzögerungsleitung eine Erfindung aus dem Hause Telefunken sei. "Es ist also gerade so, daB das SECAM-System von dem genannten, allerdings längst erloschenen Telefunken-Patent abhängig ist.« In: Archiv des Deutschen Museums München, NachlaB Walter Bruch, Signatur NL 101, Nr. 202. 
Abb. 10: "Einem son-dit zufolge geht SECAM auch noch zu Bruch", Karikatur yom November 1967, aus: Archiv des Deutschen Museums München, Nachlaß Walter Bruch, Signatur NL 101, Nr. 123.

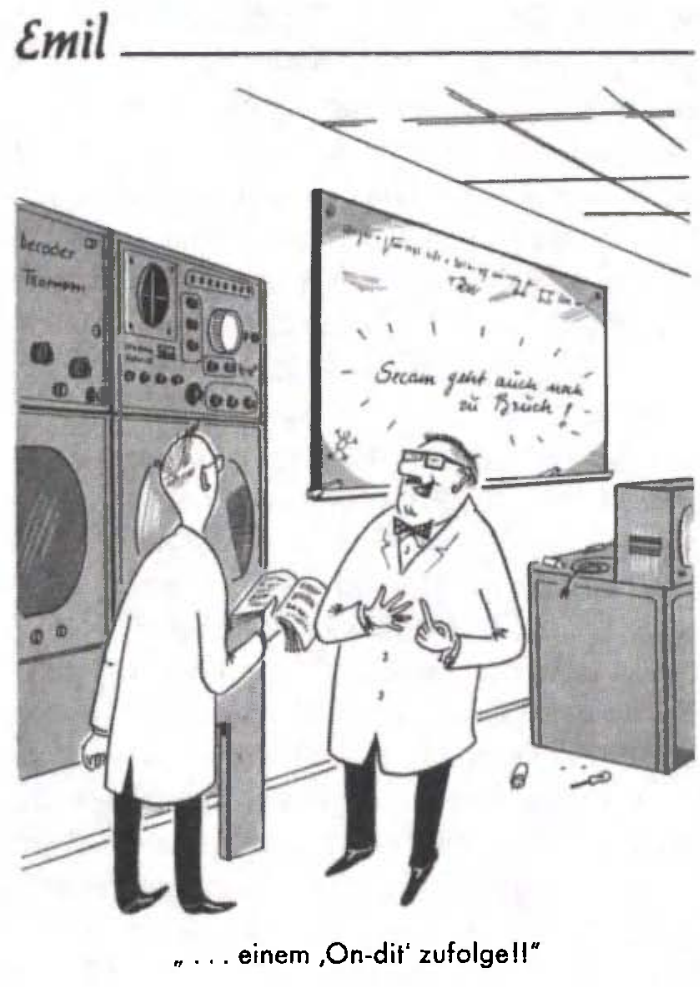

november 1966

\subsubsection{SECAM und PAL: Erfindungskontexte und Entwicklungsakteure im Vergleich}

Ohne im folgenden auf die reichhaltige Forschungsliteratur zum Thema "historische Innovationsforschung « oder Technikgenese eingehen zu können, scheint es ratsam, die soeben geschilderten Entwicklungsphasen der SECAM- und der PAL-Technologie bis zu ihrem Eintreten in die Phase der ernsthaften europäischen Standardisierungsbemühungen zu rekapitulieren ${ }^{242}$.

242 Siehe als anregenden Beitrag zu der Frage, was historische Innovationsforschung sein kann, was sie leisten könnte und was ihr als methodisches Instrumentarium zur Verfügung steht den Beitrag von Karl H. MüLLER, Wie Neues entsteht, in: Österreichische Zeitschrift für Geschichtswissenschaft 11 (2000) 1 S. 87-128. Einen knappen, aber gut strukturierten und prägnanten Überblick über den Stand der historischen und soziologischen Innovationsforschung bietet Holger BRAUN-ThürmanN, Innovation. Bielefeld 2005. Einen Überblick über die interdisziplinäre und dennoch stark wirtschaftstheoretisch dominierte Forschungslandschaft im Bereich der Innovationsstudien bietet Jan FAGERBERG, Innovation. A Guide to the Literature, in: Ders. (Hg.), The Oxford Handbook of Innovation, Oxford 2004, S. 1-26. Innovativere Ansätze, die sich verstärkt der Rolle des Konsumenten im InnovationsprozeB annehmen, tauchen in Fagerbergs Darstellung 
Für die beiden zentralen Akteure des hier geschilderten Entwicklungsprozesses war, wie bereits mehrfach betont, nicht die Erfindung eines gänzlich neuen Farbfernsehsystems die ursprïngliche Motivation ihrer Forschungsaktivität. Vielmehr ging es beiden darum, ein vorhandenes System, dessen »Genialität« von keiner Seite in Frage gestellt wurde, zu verbessern. In der evolutionären Logik von Thomas Hughes' Konzept der »large technological systems" handelt es sich demnach um "konservative " Innovationen, um Weiterentwicklungen der »radikalen« Innovation namens NTSC. Der Wunsch nach Optimierung des NTSC-Systems speiste sich aus unterschiedlichen Quellen.

Zum einen galt es, das für amerikanische Verhältnisse ausgelegte System ( 525 Zeilen bei einer Bildwechselfrequenz von $30 \mathrm{~Hz}$ ) auf seine Anwendbarkeit im europäischen Kontext zu untersuchen. Wie gesehen bauten die frühen Bemühungen der Farbfernsehentwicklung in Frankreich Mitte der fünfziger Jahre auf der Basis eines hochauflösenden s/w-Zeilenstandards auf. Das von Henri de France vorgestellte Farbfernsehsystem war mit seiner auf 409 Zeilen ausgelegten Zeilenzahl mit der bestehenden 819-Zeilennorm des Schwarzweißfernsehens kompatibel. Es waren die engen Kontakte zwischen der CSF und Telefunken im Bereich der Forschung und Entwicklung (crosslicensing-Abkommen), die dazu führten, daß sich Walter Bruch im Telefunken Grundlagenlaboratorium in Hannover ab 1958 intensiv mit dem SECAM-System beschäftigte. Bruch untersuchte die Anwendbarkeit des Systems für den sogenannten Gerber-Standard, das heißt für die in weiten Teilen Europas eingeführte 625-Zeilennorm für das Schwarzweißfernsehen. $\mathrm{Da} B$ beide Seiten die praktische Realisierbarkeit des SECAM-Systems in zahllosen Vorführungen demonstrierten, trug wesentlich dazu bei, das SECAM-System als mögliche europäische Alternative des NTSC-Systems shoffähig` zu machen.

Zum anderen spiegelt sich in den Bemühungen um die Weiterentwicklung des NTSC-Systems auch die höhere Qualitätsanforderung wider, welche die Europäer an die Fernsehtechnik stellten - vor allem im Bereich der Empfängertechnik. Wie gesehen hat dies auch mit kulturanthropologischen Differenzen zwischen Amerika und Europa in der Aneignung neuer Technologien zu tun $^{243}$. Dieses Qualitätskriterium muß zusätzlich vor dem Hintergrund unter-

jedoch überhaupt nicht auf. Siehe hierzu beispielhaft Eric vON HIPPEL, Democratizing Innovation, Cambridge M.A., London 2005.

${ }^{243}$ Als anschauliches Beispiel unterschiedlicher Aneignungsformen von Technik in verschiedenen kulturellen Kontexten siehe Martina HEssLER, »Mrs. Modern Woman«. Zur Sozial- und Kulturgeschichte der Haushaltstechnisierung, Frankfurt a. M. u. a. 2001 sowie Onno DE WIT, Adri DE la Bruhèze, Marja Berendsen, Ausgehandelter Konsum: Die Verbreitung der modernen Küche, des Kofferradios und des Snack-Food in den Niederlanden, in: Technikgeschichte 68 (2001) 2 S. 133-156. Für eine vergleichende europäische Perspektive siehe Hartmut KaELBLE, Jürgen KocKA, Hannes SiEgrist (Hg.), Europäische Konsumgeschichte: Zur Gesellschafts- und Kultur- 
schiedlicher Konsummodelle im Europa und in den Vereinigten Staaten der fünfziger und sechziger Jahre gesehen werden ${ }^{244}$. Viele Konsumgüter, die in den Vereinigten Staaten zu dieser Zeit bereits als reale Konsumgüter bezeichnet werden können, hatten in den europäischen Ländern noch den Status eines exquisiten Luxusgutes. Während der s/w-Empfänger Ende der 1950er Jahre in den USA in über $80 \%$ aller Haushalte (durchschnittliche Haushaltsgröße: 3,27 Personen) zu finden war, betrug der Anteil in der BRD knappe 20\% (durchschnittliche Haushaltsgröße 3,2 Personen). Eine Ausnahme in Europa bildete Großbritannien mit einer Fernsehgerätedichte pro Haushalt (durchschnittliche Haushaltsgröße 3,15 Personen) von über 50\% Ende der 1950 er Jahre ${ }^{245}$. In der Bundesrepublik Deutschland dauerte der Wandel vom Statussymbol zum Konsumgut beim s/w-Fernsehgerätes mit Sicherheit bis in die siebziger Jahre, beim Farbfernsehgerät gar bis Mitte der achtziger Jahre. Wie unsicher selbst Kenner der Fernsehbranche hinsichtlich der zukünftigen Entwicklung der Fernsehlandschaft noch im Jahr der Farbfernseheinführung in der Bundesrepublik 1967 waren, zeigt folgendes Zitat des Funkschau-Chefredakteurs Karl Tetzner: »Die Zeit muß zeigen, ob der Wunschtraum der Hersteller - das große repräsentative, ein Statussymbol darstellende Farbgerät ständig im Wohnzimmer und ein kleines, tragbares Schwarzweiß-Gerät von Fall zu Fall herbeigeholt - in Erfüllung geht, oder ob kleinere und tragbare Farbgeräte verlangt werden, die man zu den kurzen Farbprogrammzeiten aufstellt ${ }^{246}$. Immerhin dauerte es bis 1980 , bis die Zahl der Farbempfänger in den privaten Haushalten der Bundesrepublik zu jener der s/w-Empfänger aufschließen konnte ${ }^{247}$.

Was die Bewertung der erfinderischen oder innovativen Leistungen angeht, die in der Entwicklung des SECAM- bzw. des PAL-Systems zum Ausdruck kommen, ist eindeutig, daß die Arbeiten von Henri de France eher den Status einer innovativen technischen Lösung der Farbfernsehfrage für sich beanspruchen können als jene von Walter Bruch. Sowohl das Prinzip der sequentiellen Übertragung der Farbinformation von Zeile zu Zeile als auch die

geschichte des Konsums (18.-20. Jahrhundert), Frankfurt a.M., New York 1997. Als technikhistorisch motivierte Überblicksdarstellung siehe Wolfgang KöNIG, Geschichte der Konsumgesellschaft, Stuttgart 2000.

244 Siehe ScHILDT, SrwotTEK, Modernisierung im Wiederaufbau. Zur Konsumgeschichte in Deutschland siehe Ame ANDersen, Der Traum vom guten Leben: Alltags- und Konsumgeschichte vom Wirtschaftswunder bis heute, Frankfurt a.M. 1999.

245 Reinhard ScHNEIDER, Fernsehen in einer technisierten Welt, in: Rundfunktechnische Mitteilungen 8 (1964) 6 S. 316-322. Siehe auch Hans RindFLEISCH, Der gegenwärtige Ausbau des Fernsehrundfunks im In- und Ausland, in: Rundfunktechnische Mitteilungen 3 (1959) S. 219-227.

246 Karl TeTzNER, Die Farbe im Fernsehen: Technik - Wirtschaft - Organisation, in: Radio und Fernsehen 15 (1967) 2 S. 120.

247 Siehe Statistische Jahrbücher der Bundesrepublik Deutschland. 
Idee der Verzögerung und Speicherung dieser Information, um sie zusammen mit der Information der darauffolgenden Bildzeile zu verarbeiten, wurden international als kreative Neuerungen anerkannt. Auch der spätere Übergang zur Frequenzmodulation der Farbhilfsträger wurde von einigen Ingenieuren als technischer Fortschritt beurteilt, auch wenn damit weitreichende Folgen im Bereich der weiteren Signalverarbeitung in der Studiotechnik verbunden waren.

Im Gegensatz dazu handelt es sich bei Walter Bruchs Entwicklung des PAL-Systems nicht um eine "Erfindung « im eigentlichen Sinne des Wortes. Die Schwierigkeiten, die mit der Patentierung des PAL-Systems verbunden waren, können als Indiz für diese These gelten. Besser würde man von einer intelligenten Systemoptimierung sprechen, die selbstverständlich für sich beanspruchen kann, wichtige neue Teilkomponenten einer Netzwerktechnologie entwickelt zu haben. Wie sich später zeigen sollte, war es gerade die Nähe das PAL-Systems zum einzig in der Praxis bewährten System, dem amerikanischen NTSC-System, die sich als entscheidender Faktor in pro-PALHaltung früherer NTSC-Verfechter erweisen sollte. Dieser Durchbruch, so die These, war weniger der technischen Genialität der Systemalternative PAL zuzuschreiben als vielmehr der effektiven und konsequenten Vermarktung des Systems durch seinen Entwickler Walter Bruch.

Hier wird ein entscheidender Unterschied zwischen den beiden zentralen »Systementwicklern« Henri de France und Walter Bruch deutlich. Während Henri de France kaum auf dem internationalen Parkett zu sehen war und selbst in Frankreich das Image des Bastlers und pfeiferauchenden Grüblers besaß, welches mit der klassischen Definition eines Erfinders korreliert ${ }^{248}$, und sich diese Rolle zudem - so die Vermutung des Autors - mit der Selbsteinschätzung de France' deckte, haben wir es bei Bruch mit einem professionellen Entwicklungsingenieur eines großen Unternehmens zu tun, dessen Forschungsaktivitäten sich in fest definierten Bahnen bewegten. Ohne damit der Logik einer "path-dependancy“ von Bruchs Entwicklungsaktivitäten das Wort reden zu wollen, und darauf hinweisend, daß kaum wesentliche Unterschiede in der praktischen Arbeitsweise beider Forscher feststellbar sind, scheint dieser Umstand auf eine bedeutende strukturelle Differenz hinzuweisen. Zwar sind die Intentionen beider Forscher ähnlich - beiden geht es letztlich auch um die Durchsetzung der ihnen nach dem Patentrecht zustehenden Erfindervergütung -, doch waren ihre unternehmerischen Motivationen verschieden. Während es im Falle Henri de France außenstehende Akteure in Form der beiden Großkonzerne Saint-Gobain und CSF waren, die seine Patente aufkauften und in Form der Wiederbelebung der Compagnie Française

248 Siehe Peter Friess und Peter M. Steiner, Artur Fischer und Hans-Jürgen Warnecke sprechen über Erfindungen und Kreativität, TechnikDialog Nr. 10 des Deutschen Museums Bonn, Bonn 1996. 
de Télévision zielgerichtet zu vermarkten suchten, mußte Bruch seine Entwicklung gegen Widerstände in der eigenen Firma und in der deutschen Rundfunkindustrie behaupten.

Im folgenden kommt es deshalb darauf an, diese strukturellen Differenzen in Form unterschiedlicher Entwicklungskontexte herauszuarbeiten. Besonders wichtig erscheint vor diesem Hintergrund, die zentralen Akteure im Bereich der Rundfunkindustrie in Frankreich und der Bundesrepublik zu skizzieren. Neben den "menschlichen Akteuren«, den Entwicklungsingenieuren, Technikern und Managern, handelt es sich dabei auch um »strukturelle Akteure« (Firmen, Staaten, Standardisierungsbehörden). Beide »Akteure« müssen auf ihre Bedeutung für die Durchsetzung der Netzwerktechnologie hin untersucht werden. 
